Combination Therapy of Killing Diseases by Injectable Hydrogels : From Concept to Medical Applications

\title{
Poustchi, Fatemeh
}

2021-02-03

Poustchi , F , Amani , H , Ahmadian , Z, Niknezhad , S V , Mehrabi , S , Santos , H A \& Shahbazi , M-A 2021, ' Combination Therapy of Killing Diseases by Injectable Hydrogels : From Concept to Medical Applications ' , Advanced Healthcare Materials , vol. 10 , no. 3 , 2001571 . https://doi.org/10.1002/adhm.202001571

http://hdl.handle.net/10138/337098

https://doi.org/10.1002/adhm.202001571

unspecified

acceptedVersion

Downloaded from Helda, University of Helsinki institutional repository.

This is an electronic reprint of the original article.

This reprint may differ from the original in pagination and typographic detail.

Please cite the original version. 


\section{Combination Therapy of Killing Diseases by Injectable Hydrogels: From Concept to Medical Applications}

Fatemeh Poustchi, Hamed Amani, Zainab Ahmadian, Seyyed Vahid Niknezhad, Soraya Mehrabi, Hélder A. Santos, *Mohammad-Ali Shahbazi*

[*] F. Poustchi, Prof. H. A. Santos, Dr. M.-A. Shahbazi

Drug Research Program, Division of Pharmaceutical Chemistry and Technology, Faculty of Pharmacy, University of Helsinki, FI-00014 Helsinki, Finland

E-mail: helder.santos@helsinki.fi; m.a.shahbazi@helsinki.fi

F. Poustchi

Department of Nanotechnology, University of Guilan, Guilan, Iran

H. Amani

Department of Medical Nanotechnology, Faculty of Advanced Technologies in Medicine, Iran University of Medical Science, Hemat Highway next to Milad Tower, Tehran, Iran

Z. Ahmadian

Department of Pharmaceutics, School of Pharmacy, Zanjan University of Medical Science, 45139-56184

Zanjan, Iran

Dr. S. V. Niknezhad

Burn and Wound Healing Research Center, Shiraz University of Medical Sciences, Shiraz, Iran

Dr. Soraya Mehrabi

Department of Physiology, Faculty of Medicine, Iran University of Medical Sciences, Tehran, Iran

[*] Prof. H. A. Santos

Helsinki Institute of Life Science (HiLIFE), University of Helsinki, FI-00014 Helsinki, Finland

E-mail: helder.santos@ @elsinki.fi

[*] Dr. M.-A. Shahbazi

Zanjan Pharmaceutical Nanotechnology Research Center (ZPNRC), Zanjan University of Medical Sciences, 45139-56184 Zanjan, Iran

E-mail: $\underline{\text { m.a.shahbazi@helsinki.fi }}$

Keywords: Injectable Hydrogels; Multiple Therapy; Cancer Eradication; Tissue Engineering; Wound Dressing. 


\title{
WILEY-VCH
}

\begin{abstract}
The complexity of hard-to-treat diseases strongly undermines the therapeutic potential of available treatment options. Therefore, a paradigm shift from monotherapy towards combination therapy has been observed in clinical research to improve the efficiency of available treatment options. The advantages of combination therapy include the possibility of synchronous alteration of different biological pathways, reducing the required effective therapeutic dose, reducing drug resistance, and lowering the overall costs of treatment. The tunable physical properties, excellent biocompatibility, facile preparation, and ease of administration with minimal invasiveness of injectable hydrogels (IHs) has made them excellent candidates to solve the clinical and pharmacological limitations of present systems for multitherapy by direct delivery of therapeutic payloads and improving therapeutic responses through the formation of depots containing drugs, genes, cells, or a combination of them in the body after a single injection. In this review, currently available methods for the design and fabrication of IHs are systematically discussed in the first section. Next, as a step toward establishing IHs for future multimodal synergistic therapies, recent advances in cancer combination therapy, wound healing, and tissue engineering are addressed in detail in the following sections. Finally, opportunities and challenges associated with IHs for multitherapy are listed and further discussed.
\end{abstract}




\section{WILEY-VCH}

\section{Introduction}

Currently, changes in lifestyle, nutrition, physiological and environmental conditions have been conferred ever-increasing hard-to-treat diseases and socioeconomic challenges, such as cancer, cardiovascular disorders, neurodegenerative disorders, and infectious diseases around the world that are killing threats to the human beings. ${ }^{[1,2]}$ In recent decade, both clinical practice and exploratory studies have shown that a single treatment modality might be unable to effectively fight hard-to-treat diseases due to their physiological complexity. ${ }^{[3]}$ Therefore, current developments have gently shifted from a focus on monotherapy to combined or multiple therapies since the synergy of therapeutic agents or techniques give rise to ostentatious superadditive (namely "1 $+1>2$ ") therapeutic effects. ${ }^{[4]}$ Therefore, combined or multiple therapies have been proposed as an alternative approach to address the impediments of monotherapy. ${ }^{[5]}$ However, despite many favorable properties, available combined or multiple therapies possess several drawbacks that might limit their routine clinical use. For example, potentiation of some drugs for combined or multiple therapies is highly sequence dependent, and hence, their simultaneous combination fails to create synergistic effects against hard-to-treat diseases. ${ }^{[6]}$ In addition, challenges of formulation development have limited the rapid progress of multi-therapeutics. For example, Gliadel ${ }^{\circledR}$ wafer, which is a post-surgical drug delivery implant to avoid tumor recurrence, ${ }^{[7]}$ possesses several disadvantages, such as fast drug release, lack of adapting to shape and anatomy of desired site and subsequent implant dislodgement, high rigidity of the structure, and thus, the need for big resection cavity size (especially for treatment of glioblastoma) ${ }^{[8]}$ Therefore, new platforms should be designed to facilitate combined loading of drug molecules into the implants to, for example induce antibacterial effect and antiinflammatory properties while killing cancer cells after surgical cancer tissue resection. ${ }^{[9]}$ In this 


\section{WILEY-VCH}

context, designing new injectable platforms with adhesion properties and ability to support smart and sustained release of therapeutic molecules is highly needed. ${ }^{[10]}$

The realization, specificity, and durability of combined or multiple therapies to create favorable synergistic effects strongly depend on the effective integration of multiple therapeutic molecules in a single platform rather than just simple mixing. ${ }^{[1]}$ Currently, rapid advances in nanotechnology and biomaterials have provided high possibility for assembling several types of drugs, growth factors, and particles into a platform via chemical interactions or simple physical adsorption without the loss of bioactivity. ${ }^{[12]}$

Hydrogels are three dimensional (3D) networks of cross-linked polymer chains that are capable of absorbing significant amounts of water and biological fluids and act as potential platforms for controlled drug delivery and as an artificial extracellular matrix (ECM) for tissue engineering purposes. ${ }^{[13]}$ Besides, some polymeric hydrogel systems have shear thinning and self-recovering properties or are capable of being in-situ formed within the body after injecting in a liquid form. ${ }^{[14]}$ These properties provide the chance of preparing injectable hydrogels $(\mathrm{IHs})^{[15,16]}$ that are promising candidates in cancer therapy, cartilage repair, $\left.{ }^{[17,} 18\right]$ angiogenesis and vascularization, ${ }^{[19,20,21]}$ tissue engineering, ${ }^{[22]}$ nervous system repair, ${ }^{[19]}$ therapeutics delivery, ${ }^{[23,}$ ${ }^{24]}$ wound healing, ${ }^{[25,26]}$ etc. The in situ-forming hydrogels can be fabricated through physical interactions (ionic and hydrophobic interactions as well as supramolecular chemistry), non-toxic chemical crosslinkers, or biological cross-linking by enzymes. ${ }^{[27]}$ The outstanding properties of IHs, such as easy to handle and use, minimal invasiveness, the ability for simple and long term cell and growth factor encapsulation in the absence of chemical cross-linking, shape adaptability, proper adherence to the surrounding tissues during in situ formation, as well as improving patient compliance, make them excellent candidates for biomedical applications. ${ }^{[16,28]}$ 


\section{WILEY-VCH}

The idea of modifying and optimizing the IHs for multiple therapies is new and in the infancy stage. The unique structure of IHs allows efficient entrapment of high payloads of different therapeutic agents, protection of them from enzymatic degradation, and controlled release of them in the site of interest. ${ }^{[1,29]}$ In this review, we discuss methods for the fabrication and characterization of IHs and describe recent advances of them for multitherapy of cancer, wound healing, and other diseases (Scheme 1). Finally, we highlight future outlooks and main challenges in the development of IHs for multitherapy of various diseases.

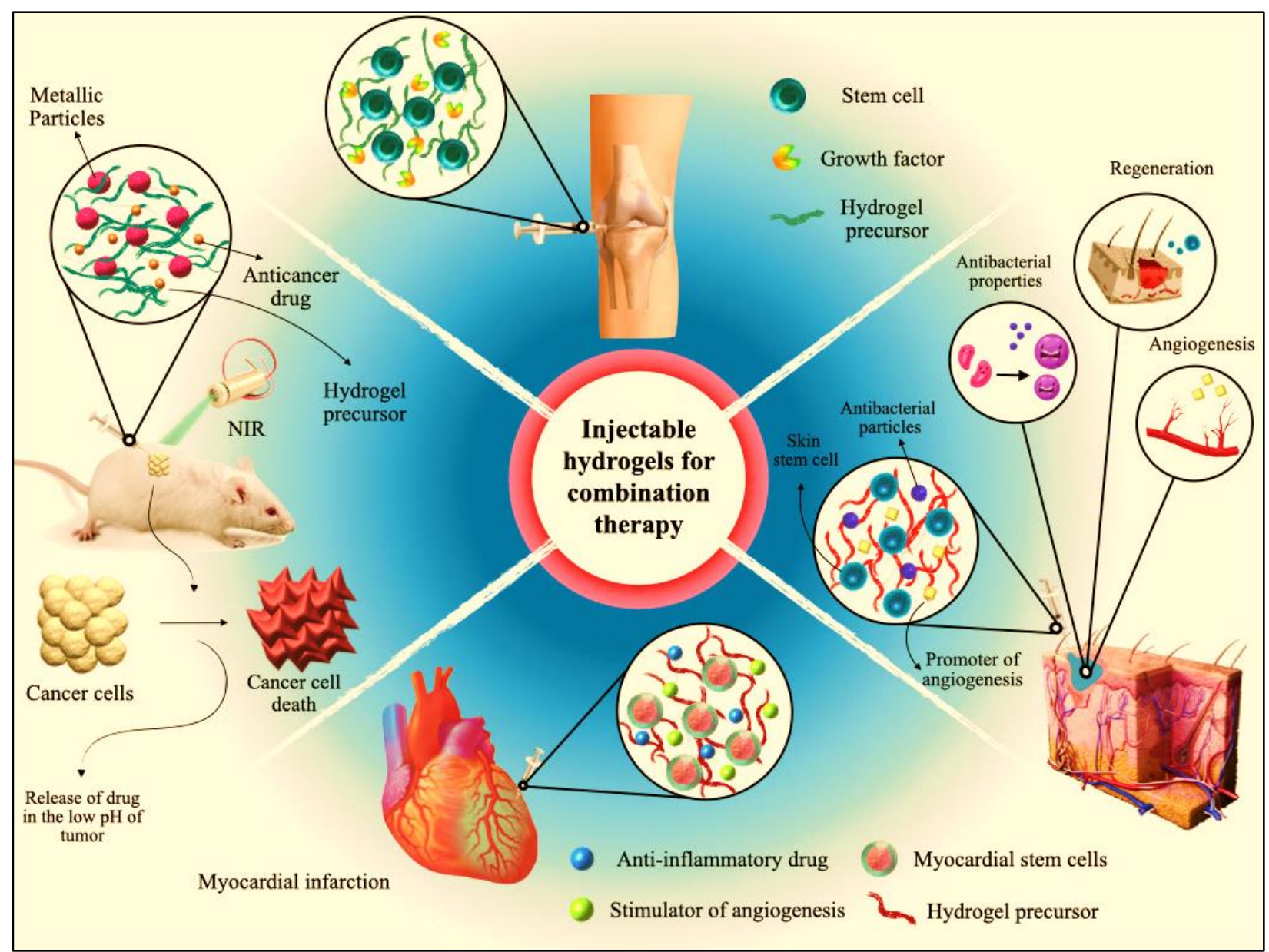

Scheme 1. Illustration of potential application of multifunctional injectable hydrogels for cancer multitherapy as well as regeneration after tissue damage.

\section{Preparation methods of IHs}




\section{WILEY-VCH}

In general, the IHs can be prepared by chemical or physical cross-linking methods. The physical cross-linking can occur through the secondary forces in which the non-covalent interactions lead to the formation of the polymeric networks without using the chemical stimuli for hydrogel formation. The non-covalent interactions, such as hydrophobic interactions, hydrogen bonding, ion cross-linking, and host-guest interactions, have been employed to design the physically cross-linked IHs. Although the physically cross-linked IHs are biocompatible with negligible cytotoxicity for human's health, they usually suffer from mechanically unstable characteristics that arise from temporary junctions among the chains. In contrast, chemically cross-linked IHs have been prepared by the formation of new covalent bonds between polymer chains, which leads to permanent and stable junctions with high stability and mechanical properties. Nevertheless, the potential toxic agents, such as cross-linking monomers, photo-initiators, organic solvents, or catalysts that might be employed for the formation of this type of hydrogel, can limit their in vivo applications. ${ }^{[30]}$ Different methods are reported in the literature for chemical cross-linking. ${ }^{[31]}$ These methods lead to the formation of hydrogels with high mechanical properties, which can be utilized to prepare the IHs with long-lasting features. ${ }^{[32]}$ Overall, the typical cross-linking strategies may have not the potential to be specific for getting injectable gels but they are also suitable for the fabrication of ordinary hydrogels. The molding of bulk gel or the injectability/mass transfer strongly can be controlled by the gelling kinetics.

\subsection{Physical cross-linking strategies}

\subsubsection{Ionic cross-linked hydrogels}

Ionic cross-linked hydrogels can be prepared by mixing ionizable polymers with counter-ions. The injectability of such hydrogels can be tuned by changing the concentration of ions, the 


\section{WILEY-VCH}

concentration of gelators, and alteration of electrical density, $\mathrm{pH}$, and temperature. Likewise, the mechanical strength of the structure can be adjusted by the cross-linking density and the molecular weight of the polymers. Some macromolecules, such as alginate can be cross-linked through the addition of cationic donors, such as calcium chloride, to them owing to high amounts of carboxyl groups in their structure. Another strategy to create an ionic cross-linking hydrogel is the combination of oppositely charged polyelectrolytes, such as sodium alginate, polylysine, and hyaluronic acid (HA) ${ }^{[33]}$ A major limitation of this strategy is the violent interaction of two oppositely charged polyelectrolytes and subsequently the formation of an inhomogeneous mixture with large aggregates. ${ }^{[34]}$ To address this challenge, $\mathrm{Wu}$ et al. used a static mixing system to prepare polyelectrolyte complex (PEC) IHs consisting of polyglutamate and chitosan $(\mathrm{CS}){ }^{[34]}$ This same team added spherical hydroxyapatite to PEC IHs through a mild gelation method and found its high capacity for injection via a $27 \mathrm{G}$ needle and improvement of soft tissue regeneration. ${ }^{[35]}$

\subsubsection{Hydrophobic interactions}

The hydrophobic moieties of amphiphilic polymers are able to mediate hydrogel formation via physical interactions. ${ }^{[36]}$ The amphiphilic polymers can be prepared by several methods, such as block or random copolymerizations, and decoration of hydrophilic macromolecules with pendant hydrophobic moieties. ${ }^{[37]}$ To ensure the injectability, the polymeric amphiphiles with upper critical solution temperature (UCST) or lower critical solution temperature (LCST) may be good choices since the change in environmental temperature results in sol-gel transition and subsequently hydrogel formation. ${ }^{[37]}$ It should be noted that a critical temperature of such 


\section{WILEY-VCH}

polymers should be chosen close to the body environment sine their gelation condition depends on temperature. ${ }^{[38]}$

\subsection{3. $\pi-\pi$ stacking interaction}

The $\pi-\pi$ stacking bonds can be created through the interplay between relatively $\pi$-electron-rich groups and $\pi$ electron-deficient groups of aromatic compounds. The $\pi-\pi$ stacking interaction can be classified in to three types based on the geometry of aromatic interactions: i) face-to-surface stacked, ii) edge-to-face stacked (T-shaped), and ii) offset stacked. ${ }^{[39]}$ For $\pi$ - $\pi$ stacking interactions in hydrogels, two modes, including plane-to-plane and edge-to-plane, have been reported. ${ }^{[40]}$ Owing to the water-rich structure of hydrogels, amino acids, such as tyrosine, tryptophan, and phenylalanine may be good choices to facilitate $\pi$ - $\pi$ stacking in hydrogels. Therefore, using gelling materials containing pre-designed peptides can ensure the formation of IHs via $\pi-\pi$ stacking interaction. ${ }^{[41]}$

\subsubsection{Hydrogen bonding}

Hydrogen bonding has been proposed as a promising cross-linking strategy since it can provide the possibility to prepare IHs with thermoplasticity and self-healing properties at the same time. On the other hand, hydrogen bonding possesses dynamic nature, and its breaking occurs at high temperature. ${ }^{[42]}$ A major disadvantage of hydrogen bonding cross-linked hydrogels that might limit their application for biomedical applications is its weak stability in the water, mainly due to the hydration and the segregation of hydrogen bonding between polymer segments. To address this problem and improve the mechanical properties of hydrogen bonding cross-linked hydrogels, several solutions have been reported. These include using macromolecules with two 


\section{WILEY-VCH}

amide moieties (formation of dual hydrogen bonding structures) or using nanoclays as bioink for adjusting the viscosity of structure. ${ }^{[42-44]}$ Several functional moieties, such as 2-ureido-4-[1H]pyrimidinone (UPy), diaminotriazine (DAT), and 6-aminocaproic acid (6-ACA) can be applied to prepare hydrogen $(\mathrm{H})$ bond-based hydrogels with specific stimuli responsiveness and shear thinning properties. ${ }^{[43,45]}$

One of the promising functional moieties that holds enormous promise to prepare $\mathrm{H}$-bonding cross-linked IHs is UPy. ${ }^{[45]}$ This moiety is able to create UPy-UPy dimer with quadruple Hbonds. The outstanding properties of UPy allow the proper control of sol-gel transition of IHs through changes in $\mathrm{pH}$ and temperature. ${ }^{[45,46]}$ The 6-ACA is another functional moiety that can be used to prepare H-bond cross-linked IHs with reversible self-healing ability upon damages stimulated by $\mathrm{pH}$ alteration. Self- healing occurs based on the H-bonds between amide and carboxylic acid moieties of two pieces of hydrogels. The good self-healing capability of IHs formed by 6-ACA under acidic conditions and their excellent adhesion to fresh gastric mucosa make them suitable platforms for sustained release of therapeutic molecules in a low $\mathrm{pH}$ environment or good tissue adhesives for stomach perforations. ${ }^{[47]}$

\subsubsection{Host-guest interaction}

Host-guest chemistry is another physical cross-linking strategy in which typical host molecules, such as crown ethers, cyclodextrins, and cucurbiturils as pendent or end moieties are grafted onto polymer backbones. ${ }^{[48]}$ The host-guest chemistry has several advantages, including reversibility, seizing of therapeutic molecules by the host moieties, and avoiding burst release, which makes it an intriguing cross-linking strategy for preparing IHs towards drug delivery and tissue engineering purposes. ${ }^{[47,49]}$ On the other hand, IHs formed by host-guest interactions possess 


\section{WILEY-VCH}

mild gelation processing without the need for any cross-linking agents and without heat generation during polymerization that can hamper the denaturation of incorporated proteins and damage to encapsulated cells at the gelation site. ${ }^{[50]}$

\subsection{Chemical cross-linking strategies}

\subsubsection{Click chemistry}

Click chemistry is one of the chemical cross-linking strategies with relatively rapid kinetics. Several advantages of click chemistry strategy, such as biocompatibility, selectivity, versatility, spontaneous reactions, high reaction rates, high product yields, and high capacity for in situ gel formation have made it a good candidate for the preparation of IHs. ${ }^{[51]}$

Several modes of click chemistry, including non-alkyl carbonyl chemistry, nucleophilic ringopening reaction, thiol-ene addition, azide-nitrile addition, carbon-carbon multi-bond addition reaction, and cycloaddition reaction have been used to prepare hydrogels. ${ }^{[52]}$ A major challenge of this strategy is the necessity of the presence of an initiator and/or a catalyst for the reaction that can hamper their bioactive application. Currently, tremendous attempts have been made to apply non-catalyst/initiator reactions for the fabrication of environmentally friendly IHs. ${ }^{[53]}$ An important advantage of click chemistry is that it can be used to prepare robust high water content (ca. 90\%) hydrogels with tunable mechanical and stiffness properties. ${ }^{[54]}$

\subsubsection{Photo cross-linking}

Photo cross-linking is one of the most popular chemical cross-linking strategies to prepare IHs in which electromagnetic radiation in the UV and visible can be applied as a source to trigger crosslinking via activation of photo-initiators and production of cations or free radicals. ${ }^{[55]}$ This 


\section{WILEY-VCH}

strategy possesses three steps, including initiation (the excitation of photoinitiators and generation of free radicals), propagation (interplay with photocurable macromers and production of reactive species), and termination (the end of cross-linking and formation of threedimensional structure). ${ }^{[56]}$ The photo-crosslinked hydrogels have several advantages, such as high viability of encapsulated cells owing to mild gelation conditions and precise temporal and spatial control over the gelation process. ${ }^{[57]}$

Based on the polymerization reaction, photoinitiation can be classified into several classes, including cationic polymerization, radical photopolymerization by hydrogen abstraction, and radical photopolymerization by photocleavage. Low penetration rate of light in the gelling mixture has been proposed as a major disadvantage of this strategy ${ }^{[58]}$ Instead of using UV light, many research groups have used visible light due to higher penetration rates and lesser damages to the tissue. ${ }^{[59]}$

\subsubsection{Michael-type addition reaction}

Michael addition reaction is based on the addition of a nucleophile (electron donor), such as amine or thiols to unsaturated carbonyl compounds like aldehydes and ketones (electron receptor) under physiological condition. ${ }^{[60]}$ In the case of IHs, the percentage of electron donor or electron receptor, the concentration of polymer solution, and its molecular weight highly affect the gelling time. ${ }^{[60]}$ Michael addition with amines is not suitable for in situ formation of IHs owing to the need for high temperature and alkaline environment. Michael addition with thiols is a good choice to prepare in situ IHs since the reaction occurs spontaneously under physiological conditions. ${ }^{[61]} \mathrm{Li}$ et al. prepared thiol derivatized hyaluronan (HA-SH) as an electron acceptor and maleilated collagen (Col-MA) as an electron donor and found that their injection to the site 


\section{WILEY-VCH}

of interest resulted in the quick formation of hydrogel (rapid gelation time about $40 \mathrm{~s}$ ). ${ }^{[62]}$ Although the most commonly used electrophiles for Michael additions are acrylates, higher rates of cross-linking have been observed for vinyl sulfones and maleimides. ${ }^{[60,63]}$ Several types of the in situ forming hydrogels based on Michael addition have been designed and fabricated by Hubbell et al. that are attractive for biomedical application since they possess minimal toxicity owing to rapid formation under physiological conditions in the absence of a catalyst and without production of any byproducts. ${ }^{[64]}$

\subsubsection{Schiff base cross-linking}

Schiff base cross-linking is based on the interplay of amine and aldehyde moieties. ${ }^{[65]}$ It is also worth noting that the number of both amines and aldehyde moieties are decisive factors of the gelation time and strength of IHs prepared by this strategy. ${ }^{[57]}$ Indeed, condensation reactions between aldehyde and amine moieties result in the formation of schiff base linkages, including oximes, imines, and hydrazones. ${ }^{[66]}$ The schiff base cross-linking has advantages, including biocompatibility, reversibility, $\mathrm{pH}$-sensitivity, and simplicity that make it a suitable candidate to design smart hydrogels for biomedical applications. ${ }^{[19,26]}$ One of the disadvantages of using Schiff base cross-linking for preparing IHs is that free aldehyde moieties in the backbone of the polymer are able to react with the amine moieties found in the biomolecules and cause toxicity and undesired side effects. ${ }^{[18,67]}$

\subsubsection{Enzymatic reaction}

Enzymatic cross-linking is another chemical cross-linking strategy that facilitates the formation of protein-based IHs through a variety of enzymes (like transglutaminases, phosphatases, glucose 


\section{WILEY-VCH}

oxidase (GOx), tyrosinase, laccase, and horseradish peroxidase (HRP) extracted from animal and plant sources. ${ }^{[68]}$ The most usually used enzymes that are used to prepare IHs are transglutaminases, ${ }^{[69,70]}$ tyrosinases $^{[71]}$ and peroxidases. ${ }^{[72]}$ Transglutaminases are known as a family of thiol enzymes that contribute to formation of a covalent bond between the gcarboxamide moieties of a peptide-bound glutamine and free amine moieties of other macromolecule. The tyrosinases are copper-containing enzymes that facilitate formation of activated quinones through oxidation of phenols in the presence of $\mathrm{O}_{2}$. Then, interplay between activated quinones and amino or hydroxyl moieties through a Michael-type addition reaction results in formation of a macromolecular network. Peroxidases are another family of enzymes that mainly use $\mathrm{H}_{2} \mathrm{O}_{2}$ as a substrate. HRP is known as the most usually used peroxidase in preparation of IHs that facilitates conjugation of the phenol and aniline derivatives in the presence of $\mathrm{H}_{2} \mathrm{O}_{2} \cdot{ }^{[73]}$

Using enzymatic reaction for the preparation of IHs represents a reliable method with numerous advantages, including reaction proceeding under physiological conditions, crosslinking efficiency, and high biocompatibility. In this method, enzyme concentration is a decisive factor of the gelation time and the degradation rate of hydrogel. ${ }^{[69]}$

\section{Multiple therapy of cancer by IHs}

Despite current advances in the development of novel therapeutic approaches, survival rate of cancer patients is still low. ${ }^{[74]}$ Owing to the diversity, complexity, and heterogeneity of tumors, as well as the high costs of oncology drug development, multiple therapies through one or more therapeutic agents or through different mechanisms can be a better choice. ${ }^{[75]}$ This approach, by targeting the pathway of pathogenesis in a synergistic manner, can enhance the therapeutic 


\section{WILEY-VCH}

efficacy and reduce the drug resistance compared to the monotherapy approach. ${ }^{[76]}$ The following section reviews recent advances of multimodal synergistic therapy in overwhelming cancer metastasis and multidrug resistance using IHs.

\subsection{IHs for the co-delivery of therapeutics to fight cancer}

Controlled co-delivery of anticancer drugs is an efficient method for cancer therapy in which the IHs can play a vital role as a smart drug carrier. The works in this area have mostly concentrated on providing synergistic therapeutic effects by the effective combination of drugs and $\mathrm{IH}$ systems. In addition to the capacity of IHs for co-delivery of therapeutic molecules, they are also commonly able to deliver drugs and genes at the same time to overcome tumor growth and metastasis. Following this concept, Liu et al. ${ }^{[77]}$ developed a peritumoral IH consisting of $\alpha$ cyclodextrin $(\alpha-\mathrm{CD})$ and a positively charged amphiphilic copolymer (methoxy-poly(ethylene glycol)-b-poly(e-caprolactone)-b-poly(ethylene imine)) with folic acid (FA)-targeted group (named as MPEG-PCL-PEI-FA). In this work, co-delivering of paclitaxel (PTX) as an anticancer drug and B-cell lymphoma-2 (Bcl-2) conversion gene, Nur77, was conducted to inhibit the growth of Bcl-2 overexpressed therapeutic-resistant tumors in a targeted manner. The PTX was loaded within the hydrophobic section of the carrier and Nur77 was adsorbed through electronic interactions with the cationic section. The results indicated that the peritumoral injection of MPEG-PCL-PEI / $\alpha$-CD supramolecular hydrogel significantly inhibited the tumor growth through dual payload release in a sustained manner (for seven days), mainly attributed to slow biodegradation of IH. More interestingly, the MPEG-PCL-PEI-FA / $\alpha$-CD supramolecular hydrogel with simultaneous administration of Nur77 gene and PTX drug through the controllable release of drug and plasmid deoxyribonucleic acid (pDNA) and targeting ability mediated by FA 


\section{WILEY-VCH}

ligand could outstandingly inhibit the in vivo expression of $\mathrm{Bcl}-2$ protein overexpressed in drugresistant liver and lung cancer cells as well as subcutaneous tumor growth at 7 days after treatment (much better performance than the mere PTX loaded IH) (Figure 1A and 1B). ${ }^{[77]}$

A major challenge associated with and the application of biomaterials for cancer therapy is the sustained release of both hydrophilic and hydrophobic therapeutic molecules from a delivery platform. This limitation has motivated researchers to develop new delivery systems such as in situ IHs that can be applied for sustained co-delivery of both hydrophilic and hydrophobic therapeutic molecules with distinct chemical and physical properties. For instance, Shen et al. ${ }^{[78]}$, designed and fabricated a thermosensitive hydrogel to deliver both hydrophilic cisplatin and hydrophobic paclitaxel (PTX). They synthesized the Bi(mPEG-PLGA)-Pt(IV) (PtGel) through linking the hydrophobic ends of two mPEG-PLGA copolymers on the Pt (IV) as a prodrug of cisplatin which could form an aqueous solution with a reversible thermosensitive sol-to-gel transition behavior. In addition, the core-corona micelles that were created in the water through the self-assembly of the amphiphilic cisplatin-polymer conjugates acted as a reservoir to solubilize the PTX (Figure 1C and 1D). Accordingly, an IH loaded with two drugs was synthesized. Interestingly, the PTX not only played a role in the anticancer activity but also decreased the sol-gel transition temperature of the IH and increased the gel strength. The in vitro experiments indicated that these drugs could be controllably released in a long period of 2.5 months, which could bring about a synergistic anticancer effect on the ovarian cancer cells. ${ }^{[78]}$ They further stated that injection of the hydrogel containing the two drug cocktail in a SKOV-3 ovarian cancer xenograft mouse model resulted in very lower tumor weight after 4 weeks in comparison with either drug alone. 

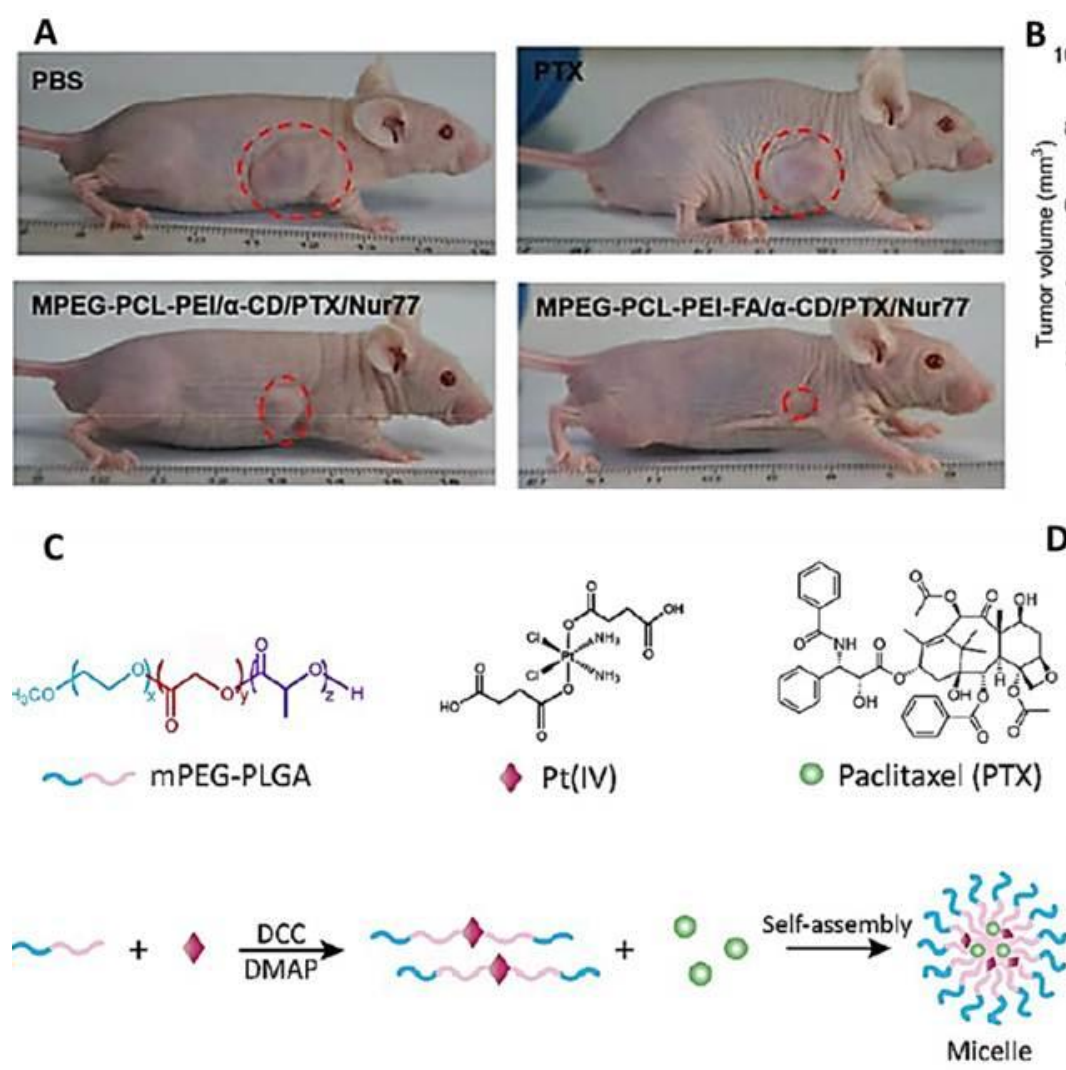
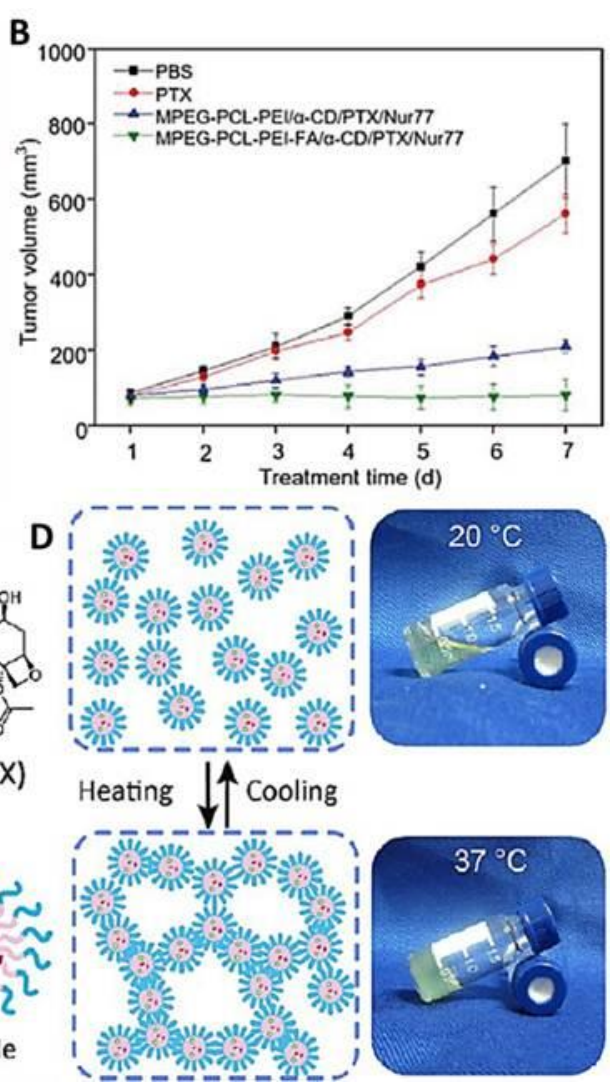

Figure 1. A) Representative Images of mice with subcutaneous tumors after treatments with phosphate buffered saline (PBS), PTX, MPEG-PCL-PEI/ $\alpha$-CD with co-loading of PTX and Nur77 gene, and MPEG-PCL-PEI-FA/ $\alpha$-CD supramolecular hydrogel with co-loading of PTX and Nur77 gene for 7 days. B) Tumor volume growth curve at day 7 after treatments with PBS, PTX, MPEG-PCL-PEI/ $\alpha-C D$ with coloading of PTX and Nur77 gene, and MPEG-PCL-PEI-FA/ a -CD supramolecular hydrogel with coloading of PTX and Nur77 gene. IHs composed of MPEG-PCL-PEI-FA/ $\alpha$-CD supramolecular hydrogel with co-loading of PTX and Nur77 gene exhibited the greatest reduction of tumor volume. Reproduced with permission. ${ }^{[7]}$ Copyright 2019, Wiley-VCH. C) Schematic illustrations of the synthesis of polymer-Pt(IV) conjugate through linking between two mPEG-PLGA diblock copolymers and a Pt(IV) prodrug and the self-assembly of the resultant conjugates into micelles. D) Sol-gel transition behavior of the PtGel upon heating in water via self-assembly into micelles at $37^{\circ} \mathrm{C}$. Reproduced with permission. ${ }^{[78]}$ Copyright 2019, American Chemical Society.

Furthermore, He et al. ${ }^{[79]}$ developed an IH to deliver three drugs, including sorafenib (SFN), doxorubicin (DOX), and metformin (MET) simultaneously with different mechanisms to combat cancer (Figure 2A). To this end, the self-crosslinking IH was made of thiolated HA derivative (HA-SH). The in vitro experiments on this triple drug-loaded IH indicated that it had cytotoxic role against the breast cancer cells (4T-1, MCF-7, and MDA-MB-231 cells) and the synergistic 


\section{WILEY-VCH}

effects of the drugs resulted in a promoted tumor cell apoptosis compared to single drug-loaded $\mathrm{IH}$. In addition, the ex vivo histological analysis of main organs of Balb/c mice clarified that this triple drug-loaded IH (Gel + DOX/SFN/MET) has less cytotoxicity compared to PBS, Gel, free drug-treated groups, Gel + DOX and Gel + DOX/SFN groups and only slight lesions could be observed only in the kidney of the Gel + DOX/SFN/MET treated groups (Figure 2B). Moreover, the Gel + DOX/SFN/MET treated group prevented the tumor metastasis due to the synergistic effects of these drugs. ${ }^{[79]}$ In another attempt, Yavvari et al. ${ }^{[80]}$ designed CS-catechol (CS-CAT)based drug-loaded IH (CAT-Gel) utilizing catechol-Fe (III) coordinative cross-linking to treat the murine lung and breast cancer models. This injectable polymeric drug carrier with selfhealing property showed very low hemolytic and cytotoxic activities. They efficiently loaded hydrophilic DOX hydrochloride and hydrophobic docetaxel (DTX) into the IH, mainly attributed to the amphipathic nature of the polymer. The gel could load DOX by $2.5 \mathrm{wt} . \%$ and the DTX by 25 wt. $\%$, separately. As for co-loading, DOX was used by $2.5 \mathrm{wt} . \%$ and DTX by $12.5 \mathrm{wt} . \%$ since the higher amount caused a negative effect on the gel-forming ability (Figure 2C). The rheology studies verified self-healing capability. After subcutaneous injection of the drug-loaded CAT-Gel into mice, the controlled release of the encapsulated anticancer drugs of DOX and DTX by synergistic effects improved the median survival against the murine lung and breast cancer models. Likewise, bioluminescence images demonstrated significant inhibition of tumor growth in mice treated with DOX-DTX-Gel in comparison to the untreated group (Figure 2D). This effort could effectively introduce a novel IH for localized delivery of drugs and providing the combined effects in cancer therapy. ${ }^{[80]}$ 


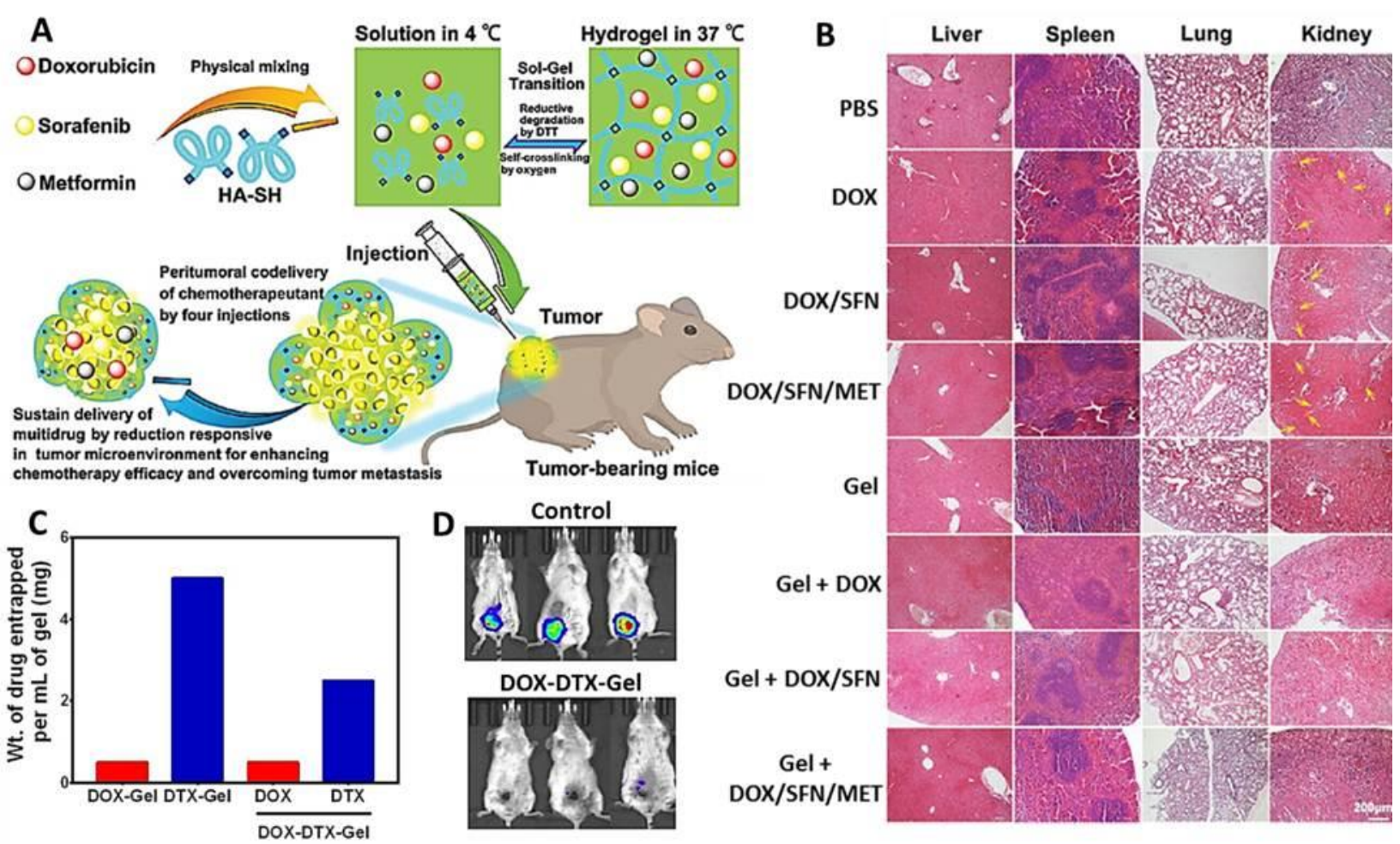

Figure 2. A) Schematic illustration of combinational chemotherapy of tumor-bearing mice through localized multi co-delivery of several drugs including SFN, DOX, and MET by an injectable selfcrosslinking hydrogel. B) The $\mathrm{H} \& \mathrm{E}$ staining of liver, spleen, lung, and kidney organs harvested from tumor-bearing Balb/c mice with various treatments (PBS, , DOX, DOX/SFN, DOX/SFN/MET, Gel, Gel + DOX, Gel + DOX/SFN, and Gel+ DOX/SFN/MET) after 16 days. The drugs, including DOX, SFN, and MET, were applied with the final concentration of 2,20 , and $20 \mathrm{mg} / \mathrm{kg}$, respectively. Gel + DOX/SFN/METshowed less cytotoxicity compared to PBS, Gel, free drug-treated groups. The sites of lesion and tissue damage were demonstrated by yellow arrows. The scale bar $=200 \mu \mathrm{m}$. Reproduced with permission. ${ }^{[79]}$ Copyright 2017, The Royal Society of Chemistry. C) Drug entrapment efficiency of DOXGel, DTX-Gel, and DOX-DTX-Gel. D) Bioluminescence images demonstrate significant inhibition of tumor growth in 4T1-12B tumor-bearing mice in DOX-DTX-Gel treated group relative to untreated group. Reproduced with permission. ${ }^{[80]}$ Copyright 2017, American Chemical Society.

Xie et al. ${ }^{[81]}$ also synthesized a CS-based IH, which was cross-linked with telechelic difunctional poly (ethylene glycol) (DF-PEG-DF) and loaded with both DOX and DTX as well as iron oxide for stimuli-responsive drug release through magnetic nanoparticle-induced hyperthermia. Using the dual-drug-loaded magnetic hydrogel and hyperthermia led to an outstanding performance in treating triple-negative breast cancer (TNBC) cells and significantly reduced tumor size. Magnetic field could regulate the release of anticancer drugs and led to the combined therapeutic 


\section{WILEY-VCH}

action via chemotherapy and thermotherapy. In vivo results demonstrated that the co-delivery of drugs had more therapeutic effects and led to more reduction of tumor size compared to single drug-loaded IH. ${ }^{[81]}$ In another study, Davoodi et al. ${ }^{[2]}$ synthesized poly (lactic-co-glycolic acid) (PLGA 50:50) and poly (D,L-lactic acid) core-shell polymeric microparticles encapsulating cisplatin and PTX using coaxial electrohydrodynamic atomization technique. Then, they embedded them into the antibacterial IH composed of alginate-aldehyde and branched polyethyleneimine (PEI-25k) that was obtained via Schiff's base reaction for local administration of these anticancer agents to combat against TNBC (Figure 3A). Consequently, a controlled release for 45 days and a synergistic effect were observed on the MDA-MB-231 cell line, in which three mechanisms were dominated, i.e., denaturation of the DNA strands, stabilization of the microtubules, and production of the intracellular reactive oxygen species (ROS). Embedding microparticles into this IH improved the sustained release manner of drugs and decreased the initial burst release, especially in the case of cisplatin and reduced the serious toxicity of cisplatin in human patients. Moreover, the $\mathrm{IH}$ was a promising tool and a non-invasive approach for retaining the particles within the tumor cavity. In addition, microparticles/hydrogel formulation loaded with the anti-cancer agents showed better therapeutic outcomes against the aggressive tumor cells with the synergistic anti-cancer activity against TNBC compared to free-drug treatment groups, suggesting the high potential of IHs for multimodal synergistic therapy against

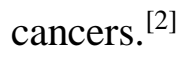

\subsection{IHs for combination therapy of cancer}

In the recent decade, radiotherapy, chemotherapy, and high intensity focused ultrasound therapy have shown great successes in the clinic for inhibition of tumor growth and a significant increase 


\section{WILEY-VCH}

in patient survival. Although other treatment options, such as immunotherapy, photothermal therapy, magnetic hyperthermia, and gene therapy are still in preliminary clinical studies, many preclinical reports have approved their high efficiency that holds great promise for future clinical translation. Nevertheless, it has been reported that single treatment modalities have failed to effectively hinder tumor growth. This section reviews multitherapies of cancers using IHs and above-mentioned treatment options.

As an example, Xing et al. ${ }^{[82]}$ reported the biomineralization triggered self-assembly of collagen protein through its mixing with chloroauric acid $\left(\mathrm{HAuCl}_{4}\right)$ in acid solution. They stated that electrostatic interaction between anionic clusters $\left(\left[\mathrm{AuCl}_{4}\right]^{-}\right)$and the positively charged collagen chains resulted in the formation of collagen protein-based hydrogels. Meanwhile, the synthesis of gold nanoparticles (GNPs) via chemical reduction of anionic clusters improved the mechanical strength of the structure, mainly attributed to the presence of GNPs as cross-linkers. In addition, the self-healing and shear-thinning characteristics were supported by the weak interactions among collagen chains and GNPs. They used collagen-based materials containing both GNPs and meso-tetra-(N-methyl-4-pyridyl) porphine tetrachloride (TMPyP) (photosensitizer) as IHs for combined photothermal therapy (PTT) and photodynamic therapy (PDT) to suppress tumor growth in the MCF-7 tumor-bearing mice. TMPyP endowed the resultant hydrogel with PDT ability and demonstrated a sustained release for $120 \mathrm{~h}$ after tumor injection. On the other hand, GNPs with excellent photothermal conversion efficiency could absorb the light and convert it to the heat that endowed the resultant hydrogel with PTT ability. Since free TMPyP suffers from fast clearance after injection, this IH could increase its bioavailability, which is highly desirable. The results revealed that the mice that were subjected to 5 times laser irradiation $(635 \mathrm{~nm}$, $169.85 \mathrm{~mW} \mathrm{~cm}^{-2}$, and $10 \mathrm{~min}$ ) were well treated, and after 23 days, the hematoxylin and eosin 


\section{WILEY-VCH}

$(\mathrm{H} \& \mathrm{E})$ staining of the tumor sections displayed no cancer cells in the vicinity of the original tumor. ${ }^{[82]}$

$\mathrm{Xu}$ et al. ${ }^{[83]}$ synthesized an in situ-forming degradable HA hydrogel for combined cancer therapy through the chemo-photodynamic route. This IH contained protoporphyrin IX (PpIX) as photosensitizer that endowed the hydrogel with PDT ability and also doxorubicin (DOX) as a chemotherapeutic agent to combat cancer via chemo-photodynamic combination therapy. For the preparation of hydrogel precursors, PpIX was chemically conjugated to adipic dihydrazide modified HA (HA-ADH) to form HA-ADH-PpIX conjugate. On the other hand, dialdehyde functionalized thioketal (TK-CHO), including a ROS-cleavable thioketal linker (TK) was synthesized as a small crosslinker. Then, the precursor solution was injected into the tumor site, and via the formation of dynamic covalent acylhydrazone between crosslinker and HA-ADHPpIX, the hydrogel (HPTG) was synthesized post-injection into the tumor site. In their study, NIR light irradiation resulted in the extensive production of ROS. The ROS not only promote efficient PDT effect but also trigger a sequential chain of events including cleavage of the ROScleavable small molecule crosslinker, inducing the degradation of IH and subsequent release of DOX molecules for cascaded chemotherapy (Figure 3B). The drug release could be adjusted by light irradiation. The $\mathrm{pH}$-responsive $\mathrm{IH}$ was further investigated in terms of its biocompatibility in which the MCF-7, 4T1 cells, and NIH-3T3 cells remained above 95\% after incubation with IHs without light irradiation that indicates high biocompatibility and favorable safety. Under a LED light irradiation $\left(633 \mathrm{~nm}, 20 \mathrm{~mW} \mathrm{~cm}^{-2}\right)$, the viability of MCF-7 cells was decreased in a time-dependent irradiation manner that confirms PDT efficacy of the formulation. The results revealed that the proposed $\mathrm{IH}$ could retain the PpIX agent for a longer time in vivo and confirmed that it could reduce the immoderate aggregation and quenching impact of the PpIX. 


\section{WILEY-VCH}

After 18 days, DOX loaded HPTG, under laser irradiation and due to the combined effect of PDT and chemotherapy, had the highest inhibition effect on the tumor growth (tumor volume and tumor weight) compared to other groups, including free DOX, HPTG with and without laser irradiation, DOX loaded HPTG without laser irradiation and control group (saline) with and without laser irradiation (Figure 3C). Combination of DOX loaded HPTG and laser therapy completely inhibited the growth of $4 \mathrm{~T} 1$ tumors, which was also verified by H\&E staining. ${ }^{[83]}$ Likewise, a time-dependent biodegradation behavior (disappearance at 35 days post-injection) was found for in situ-forming HPTG in vivo, mainly attributed to excellent biodegradability of polysaccharide HA (Figure 3D).

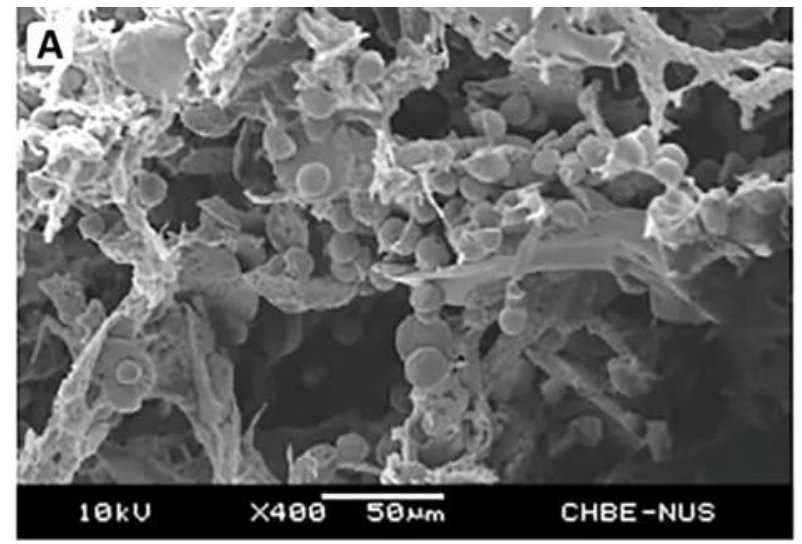

B

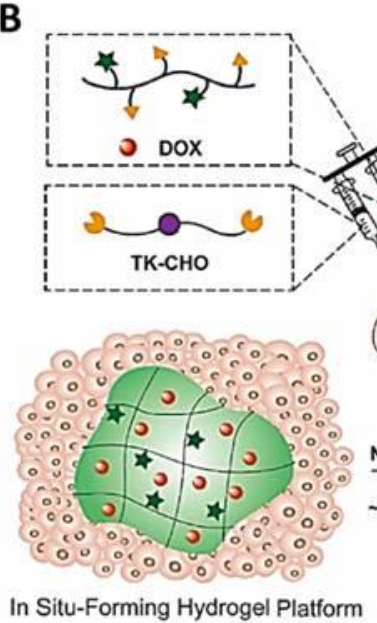

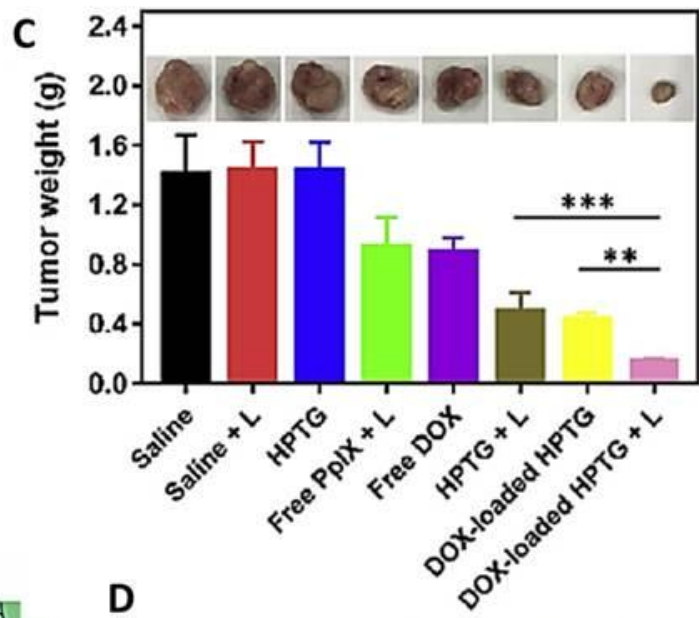

D
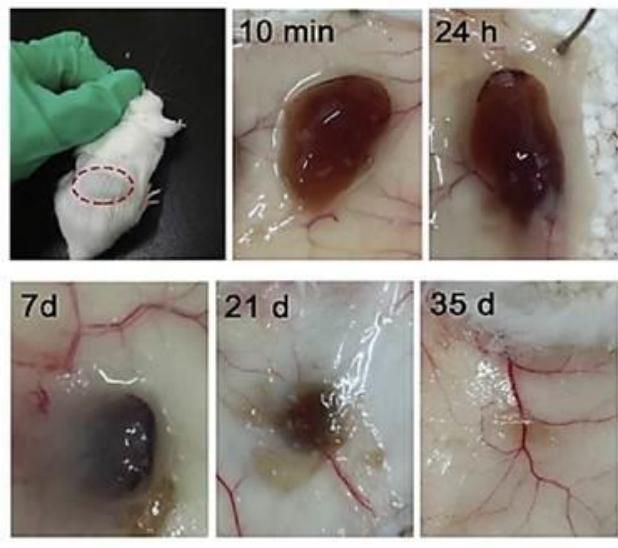

Combined Chemo-Photodynamic Therapy

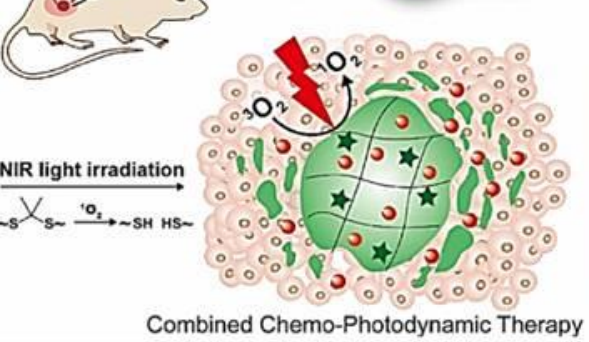

Figure 3. A) The scanning electron microscope (SEM) image of poly(lactic-co-glycolic acid) (PLGA 50:50) and poly(D,L-lactic acid) core-shell polymeric microparticles encapsulating cisplatin and PTX that were embedded into the $\mathrm{IH}$ composed of alginate-aldehyde and PEI-25k. Reproduced with permission. ${ }^{[2]}$ 


\section{WILEY-VCH}

Copyright 2017, Wiley-VCH. B) Schematic representation of injectable NIR light-triggered ROS degradable IH composed of HA-ADH-PpIX conjugate and ROS-cleavable TK-CHO for combined chemo-photodynamic therapy of tumor. C) Average weights and the typical excised tumor images for each group on the 18th day after treatment. Data are expressed as mean $\pm \mathrm{SD}(\mathrm{n}=5, * * \mathrm{P}<0.01$ and $* * * \mathrm{P}$ $<0.001)$. D) Photographs were showing excellent biocompatibility of the integrated in situ-forming hydrogel (marked with the red dashed curve) at $10 \mathrm{~min}, 24 \mathrm{~h}, 7$ days, 21 days, and 35 days post-injection. Reproduced with permission. ${ }^{[83]}$ Copyright @ 2020 Elsevier.

Gou et al. ${ }^{[84]}$ developed multi-responsive silk fibroin (SF) IHs to induce chemo/PTT /PDT therapies simultaneously. In this study, the hydrophilic SF (HSF) that was extracted from regenerated SF (RSF), was employed through the formation of $\beta$-sheet structures and hydrogenbonding interactions towards self-assembly into a hydrogel. Besides, as the photosensitizer part of this triple-therapy approach, the Cy7 was exploited, which is a near-infrared (NIR) irradiation absorbing dye with photothermal conversion capability. Furthermore, the anticancer drug DOX was encapsulated along with the Cy7 into the synthesized HSF-based IH. HSF, by the formation of $\beta$-sheet structures, endowed the hydrogel with shear-thinning, injectability, and self-healing properties. In contrast to the RSF solution, the HSF solution transformed to gel within $6 \mathrm{~h}$ at 37 ${ }^{\circ} \mathrm{C}$. The SEM images from the freeze-dried samples indicated obvious cycled morphology transformations between closely connected mesoscale multilayer structures and loosely connected multilayer structures that originated from shear-thinning stress and the following selfhealing during a resting process (Figure 4A). The gelation was not affected by DOX/Cy7 loading. The thermographic images from $\mathrm{Cy} 7$ solution and Cy7-hydrogel revealed that the photothermal ability of $\mathrm{Cy} 7$ was not reduced after hydrogel formation and after NIR irradiation for $10 \mathrm{~min}$, the temperature of $\mathrm{Cy} 7$ solution and Cy7-hydrogel increased to 55 and $56^{\circ} \mathrm{C}$ that are significantly larger than the minimum threshold temperature for tumor ablation $\left(43^{\circ} \mathrm{C}\right)$. The in vitro drug release experiments revealed that the HSF-based $\mathrm{IH}$ was sensitive to $\mathrm{pH}$ (higher release at low $\mathrm{pH}), \mathrm{H}_{2} \mathrm{O}_{2}$, and glutathione, where the acidity and $\mathrm{H}_{2} \mathrm{O}_{2}$ could efficiently 


\section{WILEY-VCH}

accelerate the DOX release rate from HSF, reaching the ultimate release of $90 \%$ in the presence of $\mathrm{H}_{2} \mathrm{O}_{2}(1 \mathrm{mM})$ at $\mathrm{pH} 6$ and after 6-day incubation (Figure 4B, 4C and 4D). The thermal infrared camera demonstrated a sharply increased temperature of tumors treated with both $\mathrm{Cy} 7$ solution or Cy7-hydrogel compared to the PBS group as a negative control at $10 \mathrm{~min}$, 48, and 98 $h$ after NIR irradiation (Figure 4E). It was found that irradiation of the NIR light with the wavelength of $808 \mathrm{~nm}$ enhanced the DOX release significantly, which verifies the lightresponsive behavior of the IH. Finally, in vivo antitumor experiments over 20 days revealed that the DOX/Cy7-hydrogel had the most inhibition rate and best therapeutic efficacy against cancer under NIR irradiation as compared to single-modal treatments (Figure 4F). ${ }^{[84]}$ 

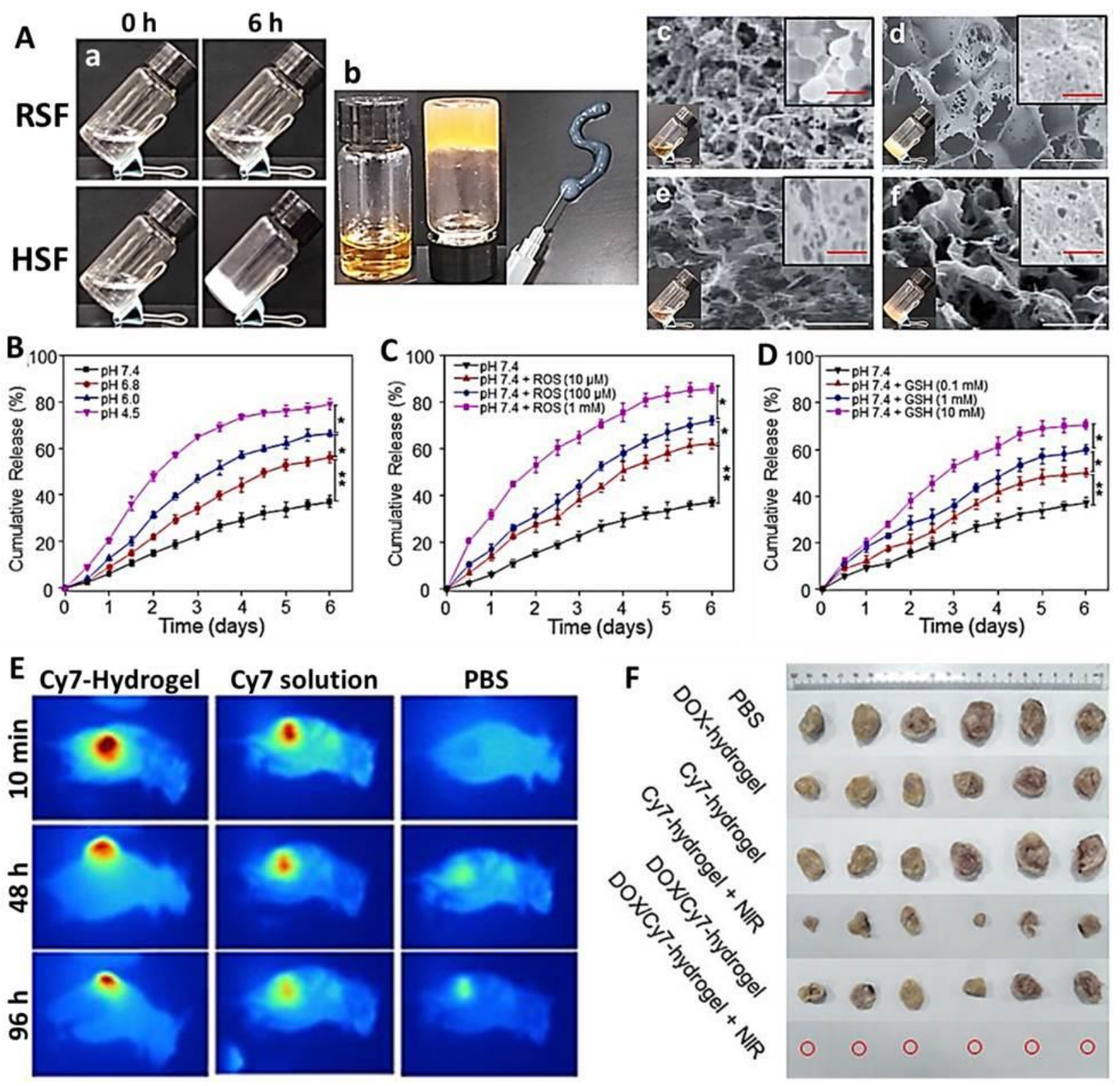

Figure 4. A) Dynamic morphology and structure of the HSF sol-gel system. (a) The photographs of RSF and HSF solution/hydrogel at $0 \mathrm{~h}$ and $6 \mathrm{~h}$. (b) The photographs of DOX/Cy7 before and after hydrogel formation. SEM images of the freeze-dried samples. (c) DOX/Cy7-HSF solution, (d) initial DOX/Cy7hydrogel, (e) DOX/Cy7-HSF shear-thinning solution, and (f) recovery DOX/Cy7-hydrogel with a resting process. The scale bars of $100 \mu \mathrm{m}$ and $500 \mathrm{~nm}$ were applied in the original images and partially enlarged images. B) The release profile of HSF hydrogel under various $\mathrm{pH}(7.4,6.8,6.0$, and 4.5) Data are expressed as mean \pm S.E.M. $(n=3)$. C) The release profile of $\mathrm{HSF}$ hydrogel under various $\mathrm{H}_{2} \mathrm{O}_{2}$ concentrations $(10,100 \mu \mathrm{M}$ and $1 \mathrm{mM})$ under $\mathrm{pH}$ 7.4. Data are reported as mean \pm S.E.M. $(\mathrm{n}=3)$. D) The release profile of HSF hydrogel under various GSH concentrations $(0.1 \mathrm{mM}, 1 \mathrm{Mm}$, and $10 \mathrm{mM})$. Data are reported as mean \pm S.E.M. $(\mathrm{n}=3)$. E) Images recorded by thermal camera showing temperature profiles of tumors at $10 \mathrm{~min}, 48$, and $98 \mathrm{~h}$ after NIR irradiation. A sharply increased temperature of tumors for both Cy7 solution or Cy7-hydrogel was seen in comparison with the PBS group as negative control at 10 min, 48, and $98 \mathrm{~h}$ after NIR irradiation. F) Representative photographs of all the tumors from different groups at the end of the experiment. The greatest reduction of tumor volume and weight were seen in 


\section{WILEY-VCH}

DOX/Cy7-hydrogel + NIR. Reproduced with permission. ${ }^{[85]}$ Copyright 2019, American Chemical Society.

Nanoparticles have also been employed to form hydrogels, as reported by Jiang et al. ${ }^{[86]}$ In their research, palladium $(\mathrm{Pd})$ nanosheets were mixed with the thiol-terminated four-arm polyethylene glycol (4arm-PEG-thiol), in which the Pd nanosheets acted as the crosslinker and an IH was achieved owing to the formation of dynamic Pd-S bonds between the Pd nanosheets and the 4arm-PEG-thiol (Figure 5A). Moreover, the DOX as an anticancer agent was incorporated into the above hydrogel. In addition, the Pd nanosheets within the IH structure, by good photothermal conversion efficiency, could readily absorb the irradiated NIR and turn it to the heat (Figure 5B). The NIR laser irradiation created combined chemo-photothermal treatment in a synergistic way to combat cancer through activation of the PTT effect of Pd NSs and stimulation of the DOX release from the hydrogel. IH could inhibit the aggregation of nanoparticles and improve their stability, while preventing their diffusing out from IH could reduce their potential toxicity. The live/dead cell staining using the 4T1 cells implied the excellent combinatorial performance of the Pd cross-linked IH in cancer therapy through controlled DOX release and hyperthermia (Figure 5C). Accordingly, the performance of this IH was evaluated in vivo over the 4T1 tumor-bearing mice, from which it was observed that the DOX loaded IH could be simply injected into solid tumors and under NIR irradiation prevented the tumor growth and also inhibited the tumor metastasis. ${ }^{[86]}$ Since the common methods for using nanoparticles as crosslinker require additional surface modification of nanoparticles, this work has a convenient preparation method without surface modification of nanoparticles and employed an effective anticancer nanosystem which can be used in further works to adopt the other 2-d nanostructures as a crosslinker to engineer the photothermal and responsive drug release behavior of the IHs. 


\section{WILEY-VCH}
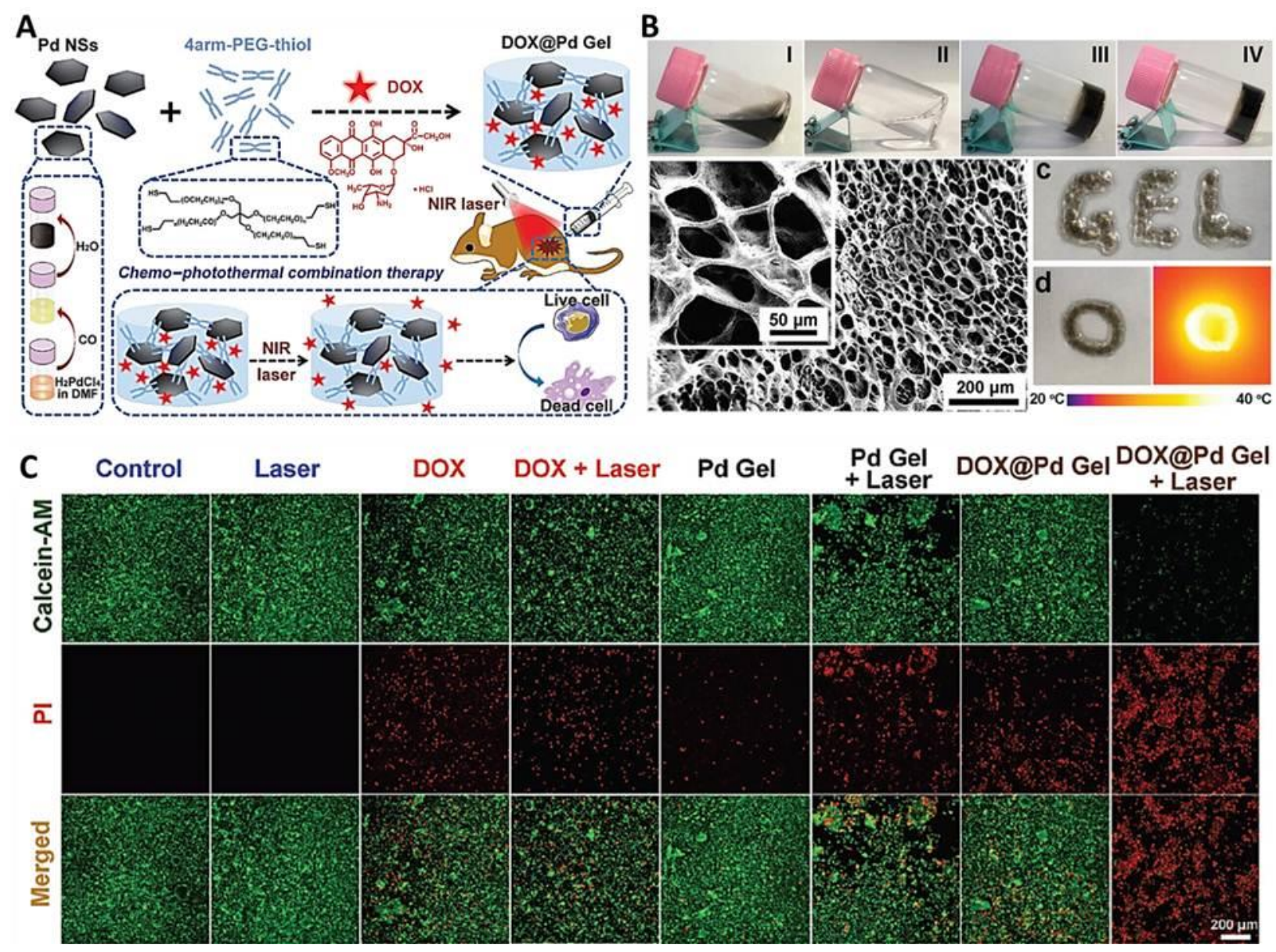

Figure 5. A) Schematic illustration of the DOX@Pd Gel synthesis process and the mechanism of combined chemo-photothermal therapy against cancer. B) Characterization of DOX@Pd Gel. (a) Images of Pd NS aqueous dispersion (I), 4arm-PEG-thiol solution (II), Pd Gel (III), and DOX@Pd Gel (IV). (b) SEM images of DOX@Pd Gel. (c) "GEL" fabricated by DOX@Pd Gel written by a syringe on a glass slide. (d) Photograph of the circle fabricated by DOX@Pd Gel and the corresponding thermal photograph was taken after laser irradiation $\left(808 \mathrm{~nm}, 0.6 \mathrm{~W} \mathrm{~cm}^{-2}, 10 \mathrm{~min}\right)$. C) Live/dead cell staining of $4 \mathrm{~T} 1$ cells. Laser: $808 \mathrm{~nm}, 0.6 \mathrm{~W} \mathrm{~cm}^{-2}$, and $10 \mathrm{~min}$. Green color: live cells; red color: dead cells. Scale bar: $200 \mu \mathrm{m}$. DOX@Pd Gel + laser resulted in a very high death of 4T1 cells than other groups. Reproduced with permission. ${ }^{[86]}$ Copyright 2020, The Royal Society of Chemistry.

Zhou et al. ${ }^{[87]}$ exploited the multistage-targeted gold/mesoporous silica core-shell nanocomposites within the HA-based IH for a combination of chemo-photothermal cancer therapy (Figure 6A). The SEM image confirmed core-shell structure of nanoparticles (Figure 6B). The core-shell nanoparticles contributed to the loading of anticancer drugs by creating suitable pores. In their study, DOX was loaded into gold mesoporous silica nanoparticles 


\section{WILEY-VCH}

(Au@MPP), and then DOX-encapsulated nanoparticles were embedded into the HA-based IH (Au@MPPD@HA ) through covalent cross-linking of the tyrosine functionalized HA (HA-Tyr) to Au@MPP under the catalysis of the HRP. Herein, phenol groups and mitochondrial targeting function triphenylphosphine were conjugated onto the surface of Au@MPP. HA fragments resided on these nanoparticles could selectively target the CD44 receptors on the cancer cell surface. Degradation of the hydrogel at the tumor site was stimulated by hyaluronidase (HAase) that was secreted by the tumor and led to Au@MPP nanoparticle release and penetration into deep tumor. Finally, the released nanoparticles selectively transported DOX into cancer cells and mitochondria. The Au nanoparticles induced the PTT behavior, and under NIR irradiation, the resultant hyperthermia accelerated the release of DOX. The in vitro experiments demonstrated that the Au@MPPD@HA was responsive to the enzyme and in the presence of HAase, the DOX release was reached to $60 \%$. However, the DOX release was only $7 \%$ after two days in the absence of enzyme. The NIR irradiation could enhance the DOX release by about $80 \%$ after 48 h. In addition, the in vivo experiments on the mice bearing MGC-803 tumors showed that after Au@MPPD@HA injection and under NIR irradiation, the local temperature reached 62 and 75 ${ }^{\circ} \mathrm{C}$ within 5 and $10 \mathrm{~min}$, while the temperature in saline and HA gel treated groups increased to $38{ }^{\circ} \mathrm{C}$ that indicated excellent photothermal conversion capability of this system. The results showed that the DOX loaded IH combined with NIR irradiation had the most inhibition effect on the tumor growth compared to other groups that indicate synergistic action of tumor targeting, chemotherapy, and PTT for cancer treatment. The histocompatibility of the IH was verified after 21 days. Moreover, the H\&E staining from normal organs showed no obvious side effects of this DOX loaded IH, while H\&E stained sections from tumor tissue revealed severe damage and necrotic death of the tumor cells in the DOX-loaded IH group under NIR irradiation. ${ }^{[87]}$ 


\section{WILEY-VCH}

In another case, Zheng et al., ${ }^{[88]}$ designed and fabricated an in-situ thermogelling CS IH containing $\mathrm{MoS}_{2} / \mathrm{Bi}_{2} \mathrm{~S}_{3}-\mathrm{PEG}(\mathrm{MBP})$ nanosheets and $\mathrm{DOX}$ for hyperthermia and chemotherapy of colon cancer. Above $37{ }^{\circ} \mathrm{C}$, due to the hydrogen bonding enhancement, electrostatic attraction, and hydrophobic interaction between the CS and $\beta$-glycerophosphate $(\beta$-GP), the solution converted from sol to gel. The incorporation of a photothermal agent with the ability to absorb NIR in the range of two biological windows including first (700-980 nm) and second (1000-1400 nm) biological windows could significantly increase the PTT capability of the system. It was demonstrated that DOX had no impact on the NIR absorption of the hydrogel. The in vivo studies revealed that the tumor surface temperature reached 45.76 and $60.43{ }^{\circ} \mathrm{C}$ after $30 \mathrm{~s}$ and 5 min under NIR irradiation $\left(808 \mathrm{~nm}, 1 \mathrm{~W} / \mathrm{cm}^{2}\right)$. Moreover, the NIR light could adjust the DOX release through local temperature increase, and due to the combination photo/chemotherapy, the tumor volume was decreased substantially after 14 days. Since the suppression of the autoimmune system as a result of cancer and the employment of chemotherapy can increase the risk of bacterial infection, the antibacterial studies against Escherichia coli (E. coli) indicated the antibacterial behavior of the CS-based IH due to inherent bacteriostatic activity of CS with the rate of $40.28 \% .{ }^{[88]}$ This antibacterial combinatorial smart system is promising for treating various cancers, and it attests to the fact that a suitable combination of photothermal nanomaterials and an IH can bring about outstanding results in killing tumor cells.

In another work, Liu et al. ${ }^{[89]}$ reported on the fabrication of an IH via host-guest interaction between PEG and $\alpha$-cyclodextrin to increase the photothermal performance, the retention time, and stability of photothermal agent, a thermosensitive photothermal network IH (PNT-gel). The PEG chains grafted on the backbone of the conjugated polymer could effectively convert the 


\section{WILEY-VCH}

irradiated NIR to light to induce the photothermal efficiency. Moreover, the reversible gel-sol transition in this thermo-sensitive IH with low critical solution transition (LCST), slightly above physiological temperature, could enhance the drug release in response to the NIR light, and as a result, an on-demand drug release could be achieved. ${ }^{[89]}$

Jin et al. ${ }^{[90]}$ engineered the polypeptide and hollow gold nanoshells (HAuNS) for synergistic chemo-photothermal therapy of the HepG2 tumor. In their study, DOX with a positive charge and polypeptide $\mathrm{PC}_{10} \mathrm{~A}$ with negative charge were coated layer by layer on the surface of negatively charged $\mathrm{HAuNS}$ to prepare $\mathrm{PC}_{10} \mathrm{~A} / \mathrm{DOX} / \mathrm{HAuNS} \mathrm{IH}$, so that one part of the DOX was adsorbed on the HAuNS surface and the other part incorporated into the $\mathrm{PC}_{10} \mathrm{~A}$ hydrogel that led to sustained drug release. $\mathrm{PC}_{10} \mathrm{~A} / \mathrm{DOX} / \mathrm{HAuNS} \mathrm{IH}$ could be readily injected through a $0.25 \mathrm{~mm}$ needle. This non-toxic hydrogel could kill the tumor cells by photothermal treatment, and the DOX release could suppress and remove the residual tumor cells. ${ }^{[90]}$ The monitoring of IR783 fluorescence demonstrated that the fluorescence intensity was quickly decayed on the first day and almost vanished on day 6 in the control group injected with free IR783 solution. In the case of the $\mathrm{PC}_{10} \mathrm{~A} / \mathrm{IR} 783$ hydrogel group, a certain fluorescence signal of IR783 was found until day 30, suggesting the sustained release of IR783 of $\mathrm{PC}_{10} \mathrm{~A} /$ IR783 hydrogel in tumors (Figure 6C). Likewise, no fluorescence signal of free IR783 was seen in the tumor and the main organs except in the liver in the control group whereas IR783 in the $\mathrm{PC}_{10} \mathrm{~A}$ hydrogel was mainly seen at the site of the tumor at day 6 after injection, indicating the potential of $\mathrm{PC}_{10} \mathrm{~A}$ hydrogel as a preeminent drug carrier for long term chemotherapy without distribution to other organs (Figure 6D).

In another example, the $\mathrm{PC}_{10} \mathrm{~A}$ polypeptide was combined with $\mathrm{PTX}$ and $\mathrm{Ag}_{2} \mathrm{~S}$ quantum dots to provide the chemo/phototherapy against the SKOV3 ovarian carcinoma tumor. ${ }^{[91]}$ In this research, firstly, the $\mathrm{PC}_{10} \mathrm{~A} / \mathrm{Ag}_{2} \mathrm{~S} \mathrm{QD} / \mathrm{PTX}$ nanogels were prepared by loading the $\mathrm{Ag}_{2} \mathrm{~S}$ quantum 


\section{WILEY-VCH}

dots (QDs) and PTX into polypeptide $\mathrm{PC}_{10} \mathrm{~A}$ nanogel through ultrasonic treatment. Then, the $\mathrm{PC}_{10} \mathrm{~A} / \mathrm{Ag}_{2} \mathrm{~S} / \mathrm{PTX}$ nanogels were dissolved into the $\mathrm{PC}_{10} \mathrm{~A}$ hydrogel from which an $\mathrm{IH}$ with the capability of cancer multi-therapy was obtained. IH, through localization, can increase the residence time of nanogel. Both the in vitro and in vivo experiments confirmed the non-toxicity of this $\mathrm{IH}$. The authors stated that the $\mathrm{PC}_{10} \mathrm{~A} / \mathrm{Ag} 2 \mathrm{~S} / \mathrm{PTX}$ could effectively suppress the growth of human ovarian carcinoma cells (SKOV3 cells) and had a performance better than those of the single NIR photothermal or chemotherapy (Figure 6E). Owing to optical properties of $\mathrm{Ag}_{2} \mathrm{~S}$ QDs, fluorescence, and photoacoustic (PA) imaging were used to monitor the in vivo degradation of $\mathrm{IH}$. The fluorescence signal of the $\mathrm{Ag}_{2} \mathrm{~S}$ QD was detected up 35 days, indicating sustained degradation process of the hydrogel in vivo. Also, the PA signal at the tumor site was reduced with the increase of time (until 9 days) that was consistent with that of fluorescence imaging (Figure 6F). ${ }^{[91]}$ 
A
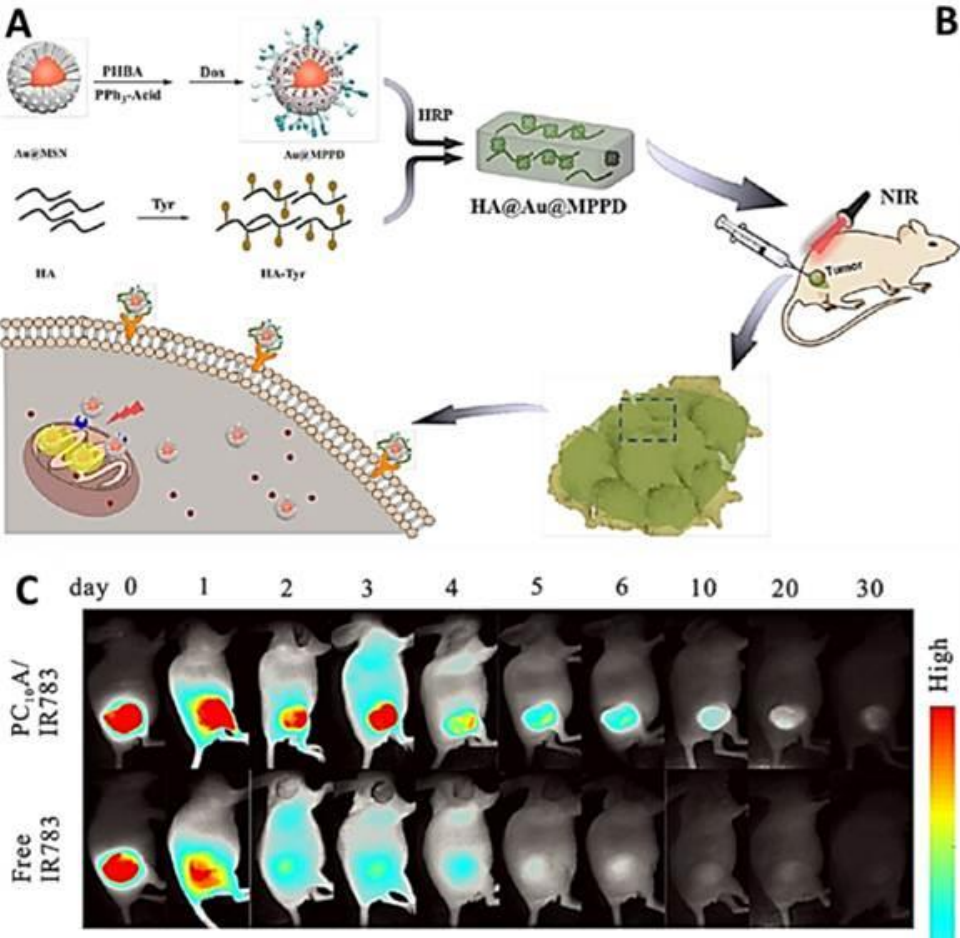

D Heart Liver Spleen Lung Kidney Intestines Tumour

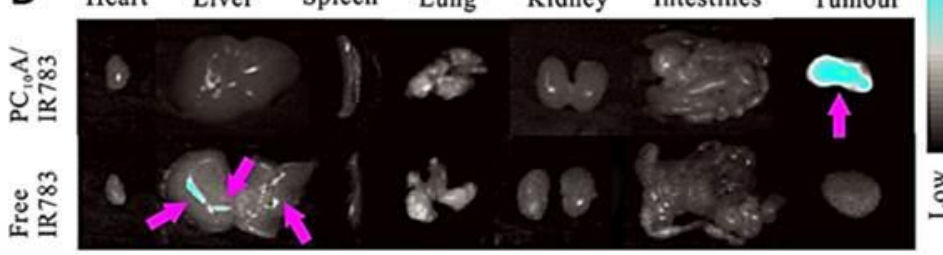

B
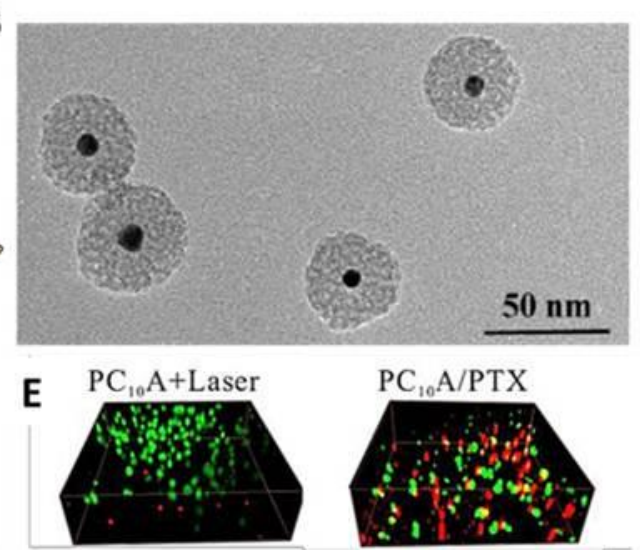

$\mathrm{PC}_{10} \mathrm{~A} / \mathrm{Ag}_{2} \mathrm{SQD} \quad \mathrm{PC}_{10} \mathrm{~A} / \mathrm{Ag}_{2} \mathrm{~S} \mathrm{QD} / \mathrm{PTX}$
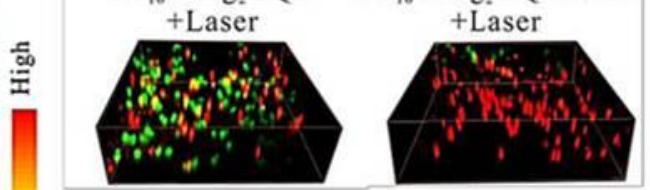

$\mathbf{F}$

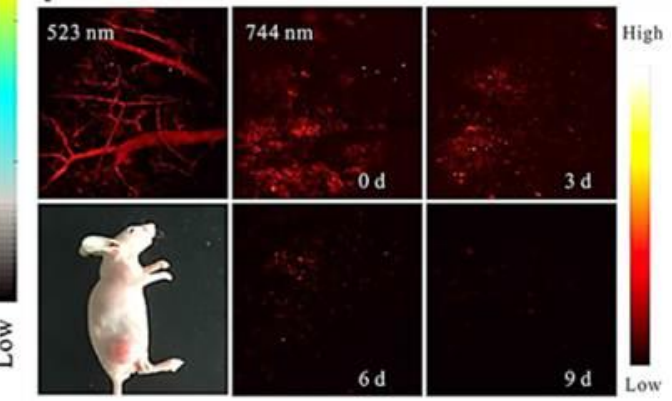

Figure 6. A) Schematic illustration of the synthesis process of multistage-targeted gold/mesoporous silica (Au@MSN) core-shell nanocomposites and its combined chemophotothermal therapy against cancer by targeting mitochondrial. B) SEM image of Au@MSN confirmed their core-shell structure. Scale bar 50nm. Reproduced with permission . ${ }^{[87]}$ Copyright 2019, American Chemical Society. C) Fluorescence images showing the $\mathrm{BALB} / \mathrm{c}$ nude mice injected with $\mathrm{PC}_{10} \mathrm{~A} / \mathrm{IR} 783$ hydrogel and IR783 solution $\left(\mathrm{PC}_{10} \mathrm{~A}\right.$ : $3 \% \mathrm{w} / \mathrm{w}$, IR783: $40 \mu \mathrm{g} \mathrm{mL}^{-1}$ ) for various time points (day $0,1,2,3,4,5,6,10,20$, and 30). A sustained release of IR783 (until day 30) was seen after its loading into the $\mathrm{PC}_{10} \mathrm{~A}$ hydrogels. D) Fluorescence images showing the main organs (heart, liver, spleen, lung, and kidney) and tumors of the mice on day 6 after injection. The $\mathrm{PC}_{10} \mathrm{~A} / \mathrm{IR} 783$ hydrogel mainly blocked into the tumor site and did not distribute to other organs. Reproduced with permission. ${ }^{[90]}$ Copyright 2019, Springer Nature. E) The cell viability of SKOV3 cells cultured in $\mathrm{PC}_{10} \mathrm{~A}$ hydrogel, $\mathrm{PC}_{10} \mathrm{~A} / \mathrm{PTX}$ hydrogel, $\mathrm{PC}_{10} \mathrm{~A} / \mathrm{Ag}_{2} \mathrm{~S}$ QD hydrogel, and $\mathrm{PC}_{10} \mathrm{~A} / \mathrm{Ag}_{2} \mathrm{~S}$ QD/PTX hydrogel $\left(\mathrm{PC}_{10} \mathrm{~A}: 3 \%\right.$ w/w, $\mathrm{Ag}_{2} \mathrm{~S}$ QD: $\left.1 \mathrm{mg} \mathrm{mL}-1, \mathrm{PTX}: 200 \mu \mathrm{g} \mathrm{mL}-1\right)$, respectively. In the $\mathrm{PC}_{10} \mathrm{~A}$ hydrogel, $\mathrm{PC} 10 \mathrm{~A} / \mathrm{Ag}_{2} \mathrm{~S}$ QD hydrogel, and $\mathrm{PC}_{10} \mathrm{~A} / \mathrm{Ag}_{2} \mathrm{~S} \mathrm{QD} / \mathrm{PTX}$ hydrogel groups, an $808 \mathrm{~nm}$ laser at a power density of $2.0 \mathrm{~W} \mathrm{~cm}^{-2}$ was applied for 5 min exposure to cells. Green color: live cells, red color: dead cells. $\mathrm{PC}_{10} \mathrm{~A} / \mathrm{Ag}_{2} \mathrm{~S}$ QD/PTX hydrogel + laser induced the greatest death of SKOV3 cells. F) The PA images showing sustained degradation process of the $\mathrm{PC}_{10} \mathrm{~A} / \mathrm{Ag}_{2} \mathrm{~S} \mathrm{QD} / \mathrm{PTX}$ hydrogel $(100 \mu \mathrm{L})$ until day 9 after injection in mice under excitation of 744 and $523 \mathrm{~nm}$. Reproduced with permission. ${ }^{[91]}$ Copyright 2019, The Royal Society of Chemistry. 


\section{WILEY-VCH}

This trend has also been followed by other researchers to simultaneously use gold nanostructures and DOX in thermoresponsive IH to provide the chemo-phototherapy. ${ }^{[92]} \mathrm{He}$ et al. ${ }^{[93]}$ synthesized a type of multi-stimuli ( $\mathrm{pH}$, enzymes, and near-infrared light)-responsive $\mathrm{IH}$ based on amphoteric gelatin nanoparticles (Gela NPs) and polydopamine nanoparticles (PDA NPs) loaded with the DOX for combined chemo-photothermal cancer treatment. PDA NPs with good photothermal conversion capability endowed the hydrogel with PTT ability. After DOX loading into PDA NPs, the Gela NPs and DOX-PDA NPs were blended at basic condition $(\mathrm{pH} \approx 12)$, then by introducing an acidifier (glucono delta-lactone, GDL), the system $\mathrm{pH}$ was reduced to less than the isoelectric point of the Gela NPs that induced the charge reversal of Gela NPs and resulted in the formation of a uniform hydrogel. This system could act as a smart DOX carrier that was responsive to acidic $\mathrm{pH}$, enzyme, and also NIR. The acidic $\mathrm{pH}$ increased the DOX release that is related to more solubility of DOX and the repulsion between positively charged Gela NPs and DOX at acidic PH. Owing to the sensitivity of GEL NPs to acidic $\mathrm{pH}$ of the tumor microenvironment and PDA NPs to the NIR laser irradiation, the IH was able to release DOX into the tumor site in a sustained and controlled manner. ${ }^{[93]}$ Some other researchers have also tried to use various IH structures along with the photothermal agents and anticancer drugs and also employed the X-ray radiation for this purpose. ${ }^{[94]}$ In addition to the NIR triggered hyperthermia, magnetic hyperthermia is another mechanism that can be used for combined cancer therapy. In this regard, Zhang and Song ${ }^{[95]}$ employed the superparamagnetic iron oxide nanoparticles (SPIONs) along with the tumor necrosis factor-related apoptosis-inducing ligand (TRAIL) to fabricate a novel cancer multi-therapy system. For this purpose, they developed an injectable, biodegradable, and thermosensitive TRAIL/SPION noncomplex hydrogels (T/S-NHs) 


\section{WILEY-VCH}

through electronic and hydrophobic interaction between positively charged TRAIL and oleic acid-coated SPIONs and negatively charged poly (organophosphazene) (PPZ). This thermosensitive IH could convert to hydrogel at the body temperature $\left(37{ }^{\circ} \mathrm{C}\right)$, and $\mathrm{T} / \mathrm{S}$ noncomplex release was increased under magnetic hyperthermia $\left(43{ }^{\circ} \mathrm{C}\right) .{ }^{[95]}$

Another trend in multitherapy of the cancer is the imaging-guided tumor ablation. In this respect, Zhao et al. ${ }^{[96]}$ designed and developed a phase-changeable alginate-based $\mathrm{IH}$ for synergistic hyperthermia and chemotherapy. They fabricated an alginate/MoS $2 / \mathrm{Bi}_{2} \mathrm{~S}_{3}$-poly (ethylene glycol) (MBP)/DOX (AMD) hydrogel through binding between $\alpha$-L-guluronic blocks of alginate and calcium ions in which the DOX and MBP nanosheets were encapsulated. Moreover, the MBP nanosheets and DOX could provide the computed tomography/PA imaging-guided tumor cell targeting through photothermal and chemotherapy mechanisms, respectively, which was proved in the in vivo experiments. This alginate-based IH could achieve the photothermal conversion efficiency of $42.7 \%$, and upon the photothermal transformation of the MBP nanosheets, the drug diffusion from the IH was improved. More interestingly, this novel IH structure could retain the encapsulated MBP and DOX and prevented their release to the bloodstream. ${ }^{[96]}$

Another mechanism of multiple cancer therapy is to combine radiotherapy and chemotherapy, through which the radioactive agents and anticancer drugs can be simultaneously loaded over the IH. Following this concept, Puente et al. ${ }^{[97]}$ aimed at the targeting of glioblastoma (GBM) in the brain, which is totally heterogeneous. They synthesized a CS-based IH with the ability of controllable releasing of the temozolomide (TMZ) as a chemotherapeutic agent, which could also retain the iodine as a radioactive isotope within its network. As declared from the in vitro results, the iodine isotopes could be retained for more than 42 days. In contrast, the TMZ was 


\section{WILEY-VCH}

completely delivered on-demand within $48 \mathrm{~h}$. This study suggested that injectable chemo-radiohydrogel implants can be served as good tools to improve local chemoradiotherapy of GBM. ${ }^{[97]}$ In addition to the investigations on targeting the cancer cells, immunotherapy can also be carried out along with the photothermal cancer treatment by IHs. For example, Mei et al. ${ }^{[98]}$ loaded the photothermal drug methylene blue and the immunological agent imiquimod (R837) within the collagen/alginate IH with shear-thinning property that was prepared by co-assembly of positively charged collagen and negatively charged alginate. The researchers found that PTT, following intratumoral injection of the collagen/alginate hydrogel resulted in the initiation of the tumorassociated antigens (TAA) related immune responses. A combination of TAA and R837, released from $\mathrm{IH}$, created a vaccine that was capable of triggering an antitumor immune response and hence effectively suppressed cancer metastasis. ${ }^{[98]}$

\section{Multi-therapeutic IHs for wound healing}

A major focus of biomaterial-related research has long been the design and fabrication of a 3D structure to manage re-epithelialization and dermal regeneration of cutaneous wounds and epithelial tissues. ${ }^{[99]}$ Numerous efforts are underway to design delivery systems, including several therapeutic molecules to cure hard-to-heal wounds. In this section, we will delineate some examples of nano-engineered IHs for combined therapy of such wounds. IHs can be applied to simultaneously deliver antibacterial agents and growth factors at the wound site that, in turn, result in better management of chronic wounds with predisposing to infection in comparison to monotherapy. For example, Qu et al. ${ }^{[100]}$ have introduced a multifunctional IH for wound healing by synthesizing N-carboxyethyl chitosan (CEC) and oxidized HA (OHA). The OHA was grafted with aniline tetramer (OHA-AT). Then, to synthesize the OHA-AT/CEC, they 


\section{WILEY-VCH}

dissolved the CEC polymer in the PBS solution, followed by the addition of OHA-AT. The hydrogel was prepared via mixing the CEC solution and the OHA-AT solutions and through the formation of the Schiff base bond between their functional groups at the temperature of $37^{\circ} \mathrm{C}$. In order to load drugs in the prepared IHs, during the synthesis process, drugs such as amoxicillin were added. It was found that the AT could enhance the mechanical properties and elongate the gelation time. In the course of drug delivery, these IHs could release up to $73 \%$ amoxicillin after $48 \mathrm{~h}$ through diffusion mechanism that led to significant antibacterial activity against Staphylococcus aureus (S. aureus) and E. coli. Interestingly, the $\mathrm{HCl}$ doped OHA-AT/CEC hydrogels were effective in scavenging the ROS, which was confirmed through examining the reduction of $\alpha, \alpha$-diphenyl- $\beta$-picrylhydrazyl (DPPH) free radicals that were scavenged around $80 \%$ in an AT concentration-dependent manner. The higher AT contents into the structure of IHs gave rise to faster wound healing processes than gels with less AT amount, mainly attributed to antioxidant activity from AT that provides electron transfer to free radicals. Moreover, IH showed anti-inflammatory, conductivity, and angiogenesis properties. Furthermore, the wound healing rate of this $\mathrm{IH}$ was much faster than the commercial counterparts, which resulted in a higher density of fibroblasts and improving collagen deposition and vessel regeneration. ${ }^{[100]}$ Owing to the impairment of vascularization, management of diabetic wounds is difficult considering their high susceptibility to microbial infection. To tackle this problem, Chen et al. ${ }^{[101]}$ synthesized an IH based on the Ag-S coordination bond between multi-arm thiolated polyethylene glycol (SH-PEG) and silver nitrate $\left(\mathrm{AgNO}_{3}\right)$. The bacteria-killing activity of $\mathrm{Ag}^{+}$ was involved in the improvement of diabetic wound regeneration. This type of cross-linking endowed the hydrogel with self-healing and injectability properties. The deferoxamine (DFO) was loaded as the angiogenic drug during cross-linking. The in vivo experiments showed 


\section{WILEY-VCH}

efficient repair of the diabetic skin wound along with improved angiogenic and antibacterial performance (Figure 7A and 7B). In addition, the assessment of the in vitro antibacterial activity via observing the clear zone of inhibition (ZOI) around the hydrogel against the $S$. aureus and the in vivo antibacterial activity through immunofluorescent staining by $S$. aureus antibodies (Figure 7C and 7D) showed that DFO loaded hydrogel could effectively inhibit bacterial growth, mainly attributed to iron chelation and subsequent amplification of the antibacterial effect of $\mathrm{Ag}^{+}$. The neovascularization was confirmed through increasing the CD31 expression (Figure 7E and $7 \mathrm{~F}$ ) and significantly increased the expression level of vascular endothelial growth factor (VEGF). Moreover, in the presence of DFO loaded IH, the re-epithelialization and in vivo wound healing ability were dramatically increased. ${ }^{[101]}$. In another study, Kong et al. ${ }^{[102]}$ loaded DFO and bioactive glass (BG) as stimulators of angiogenesis into the alginate-gluconolactone IHs to treat diabetic chronic skin defects in rats. The combination use of BG and DFO had better effects on migration and tube formation of human umbilical vein endothelial cells (HUVECs) in comparison to monotherapy either with BG or DFO. ${ }^{[102]}$ 


\section{WILEY-VCH}

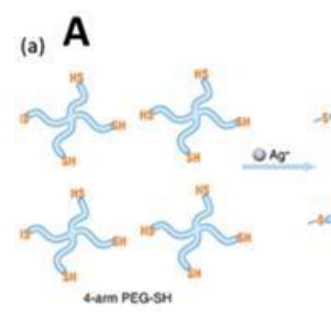

(d)

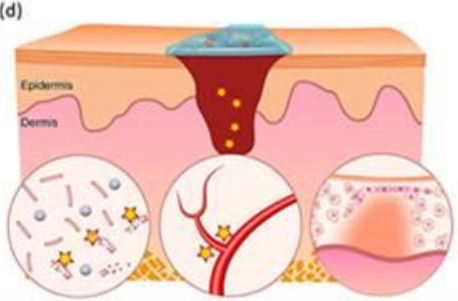

C Control

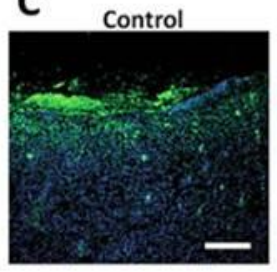

D

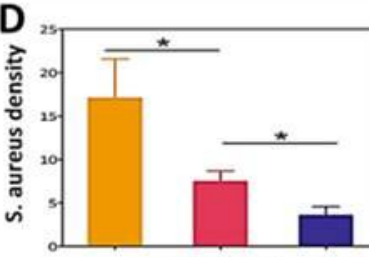

$\square$ Control (b)

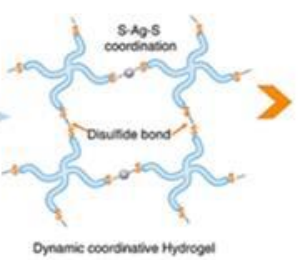

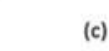

(c)
B

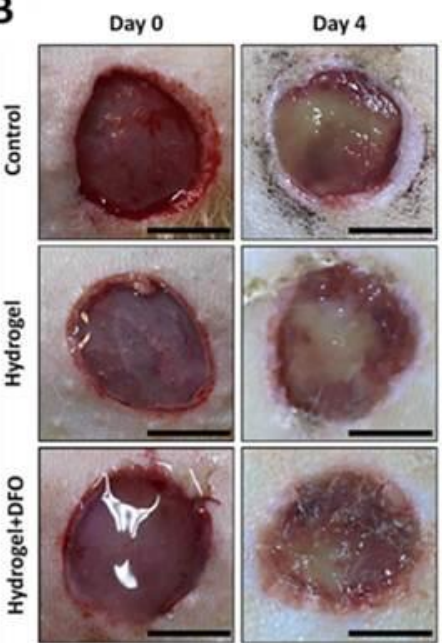

Day 7

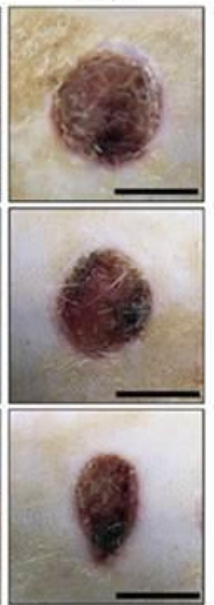

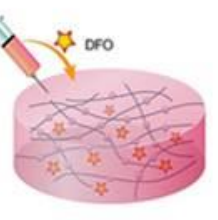

$\checkmark$

(1)

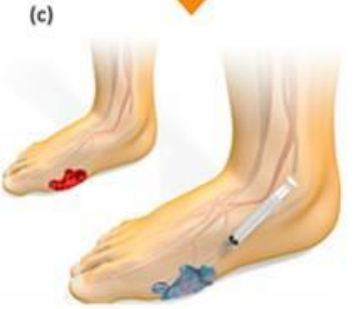

$\mathrm{E}$
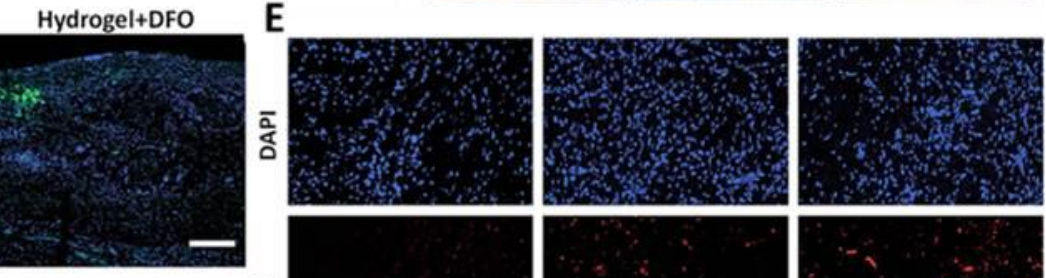

ใิ
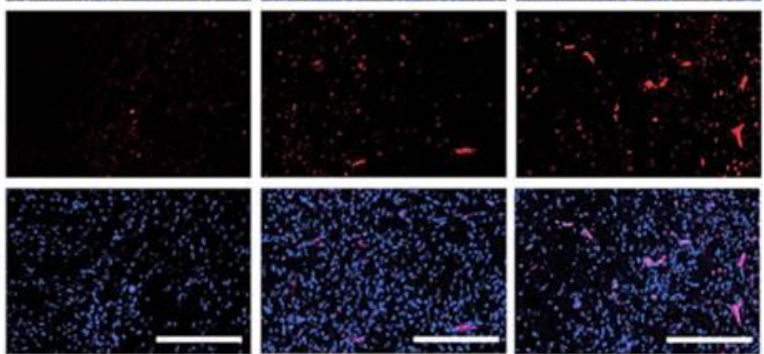

Figure 7. A) Schematic illustration of the self-healing $\mathrm{Ag}(\mathrm{I})$-thiol (Au-S) hydrogel synthesis process through mixing 4-arm-PEG-SH and $\mathrm{AgNO}_{3}$ and the application of hydrogel with injectability, antibacterial, angiogenic, and reepithelialization properties for diabetic skin wound repair. B) Representative photographs captured with a digital camera showing amount of healing in the control wounds and wounds injected with the hydrogel, and the DFO loaded hydrogel on 0, 4,7 days postinjection. Scale bars $=1 \mathrm{~cm}$. C) The in vivo antibacterial activity evaluation using immunofluorescent staining with S. aureus antibodies (green) and DAPI counterstaining (nucleus, blue). Scale bars $=100 \mu \mathrm{m}$. D) The quantitative analysis of the staining intensities of S. aureus in control, hydrogel, and DFO loaded hydrogel groups $\left({ }^{*} p<0\right.$. 05). E) Immunofluorescence staining of vascular endothelial cells treated with DFO-hydrogels with CD31 as a marker of neovascularization and the nuclei were counterstained with DAPI. Scale bars $=100 \mu \mathrm{m}$. F) The quantitative data of the staining intensities of CD31 in control, hydrogel, and DFO loaded hydrogel groups $\left({ }^{*} \mathrm{p}<0.05,{ }^{* *} \mathrm{p}<0.01\right)$. Reproduced with permission. ${ }^{[101]}$ Copyright 2019, Nature Publishing Group.

In another study, Li et al. ${ }^{[103]}$ developed a biocompatible IH using sodium alginate (SA) and the bioactive hardystonite (HS) bioceramic as a multifunctional injectable wound dressing that was 


\section{WILEY-VCH}

able to simultaneously hamper bacterial growth and promote angiogenesis and wound healing. HS was able to release $\mathrm{Ca}^{2+}, \mathrm{Zn}^{2+}$ and $\mathrm{Si}$ ions, which $\mathrm{Ca}^{2+}$ and $\mathrm{Zn}^{2+}$ ions could act as crosslinkers for hydrogel formation through double ion cross-linking. Additionally, $\mathrm{Zn}^{2+}$ ions could act as nutrition for wound healing and as antibacterial components, and $\mathrm{Si}$ ions could induce angiogenesis for improving wound healing (Figure 8A). Since the fast reaction between SA and divalent ions is an obstacle to achieve injectability, controlling the gelation process of the hydrogel was achieved through adjusting the release of the incorporated $\mathrm{Ca}^{2+}$ and $\mathrm{Zn}^{2+}$ ions from HS. Both extract assay and direct contact assay as antibacterial tests demonstrated a 100\% antibacterial rate of the SA/HS composite hydrogel while the pure SA hydrogel failed to exert antibacterial effects (Figure 8B). In addition to antibacterial activity, the in vitro experiments demonstrated that the IH was capable of promoting the proliferation and migration of both human dermal fibroblasts (HDFs) and HUVECs. On the other hand, in vivo experiments demonstrated the significant formation of epithelium and blood vessels, indicating the high potential of this IH for multiple therapies. ${ }^{[103]}$

One major limitation of the conventional IHs for co-delivery into the wound site is the lack of self-healing ability that results in their damage by external forces during clinical therapy, especially for the irregular wound sites. ${ }^{[104]}$ The damage to IHs during wound therapy might lead to function loss and increased risk of bacterial infection at the wound site. To overcome these problems, Chen et al. ${ }^{[105]}$ developed a covalent dynamic IH with the properties of self-healing, antibacterial property, and constant drug release through an interplay between aminated gelatin (NGel), oxidized dextran (ODex), and adipic acid dihydrazide (ADH). The self-healing ability of IH was attributed to the imine and acylhydrazone bonds. The basic fibroblast growth factor (bFGF)@poly(lactic-co-glycolic acid) (PLGA) microspheres and chlorhexidine acetate (CHA) 


\section{WILEY-VCH}

were incorporated into the IHs. The engineered hydrogel exhibited retained morphological integrity after swelling and excellent biodegradability. They concluded that the engineered IH was able to promote cell proliferation and wound healing through the sustained release of bFGF and burst release of CHA. ${ }^{[105]}$

In addition to the drug molecules, IHs can be used for the co-delivery of cells and therapeutics owing to their similarity to ECM. ${ }^{[106]}$ For example, Zhao et al. ${ }^{[107]}$ fabricated $\mathrm{pH}$ - and glucosesensitive IHs via the covalent crosslinking of imine bond and phenylboronate ester using benzaldehyde-capped PEG (OHC-PEG-CHO), phenylboronic-modified chitosan (CSPBA), and poly(vinyl alcohol) (PVA). Fibroblasts and insulin were loaded into the IHs during the in situ crosslinking. Owing to the stability of imine bond at physiological $\mathrm{pH}$ and its instability at acidic $\mathrm{pH}$, the system was able to release necessary factors for wound healing at mildly acidic $\mathrm{pH}$. In these engineered IHs, the diol moieties of glucose strongly bind to boronic acid derivatives via the formation of reversible boronate ester. They reported that the introduction of imine bonds (benzoic-imine bonds) and phenyl boronic acid moieties into the engineered IHs resulted in dual responsive release of insulin and fibroblasts at wound site of a streptozotocin-induced diabetic animal model. The insulin/cell-loaded IHs were able to improve collagen deposition, neovascularization, and the healing process of diabetic wounds. Dong et al. ${ }^{[62]}$ also reported cutaneous wound healing along with stem cell retention by poly(ethylene glycol) (PEG)-gelatin hydrogel. The hydrogel was prepared under Michael-type addition reaction between acrylate groups of poly (ethylene glycol) diacrylate (polyPEGDA) polymers and thiolated anions from the thiolated gelatin (Gel-SH). The gelation was induced in response to the physiological conditions in a short period of $2 \mathrm{~min}$. In this work, the murine adipose-derived stem cells (ASCs) were encapsulated into the $\mathrm{IH}$. Owing to 3D network structure and structural similarity to ECM, 


\section{WILEY-VCH}

the hydrogel was able to support cell survival, proliferation, migration as well as higher cell retention compared to direct delivery of the cells to the wound site. The bioluminescence imaging demonstrated the "hydrogel + cells" group created significantly higher bioluminescence signal in vivo up to 14 days compared to the "cells alone" group (Figure 8C). The hydrogel also showed adjustable mechanical property, biodegradability, and biocompatibility that support it for cell engraftment. The CD68 immunohistochemical staining displayed that the cell loaded IH significantly decreased the number of macrophages, which was attributed to the effective role of $\mathrm{IH}$ in wound healing. Also, the CD31 immunohistochemical staining showed that the strategy of combining cells and IH could enhance angiogenesis. Finally, the rate of wound closure in hydrogel + cells was much faster than in the other groups (Figure 8D). ${ }^{[62]}$
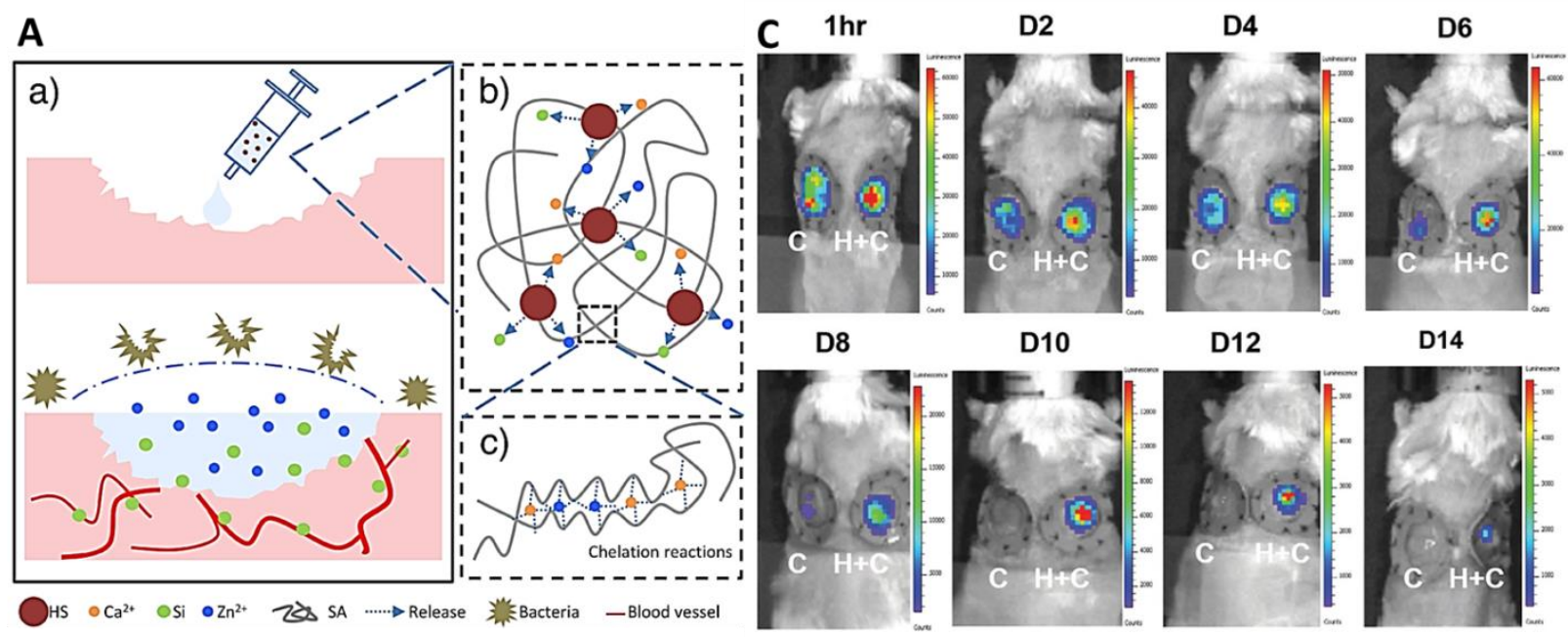

B
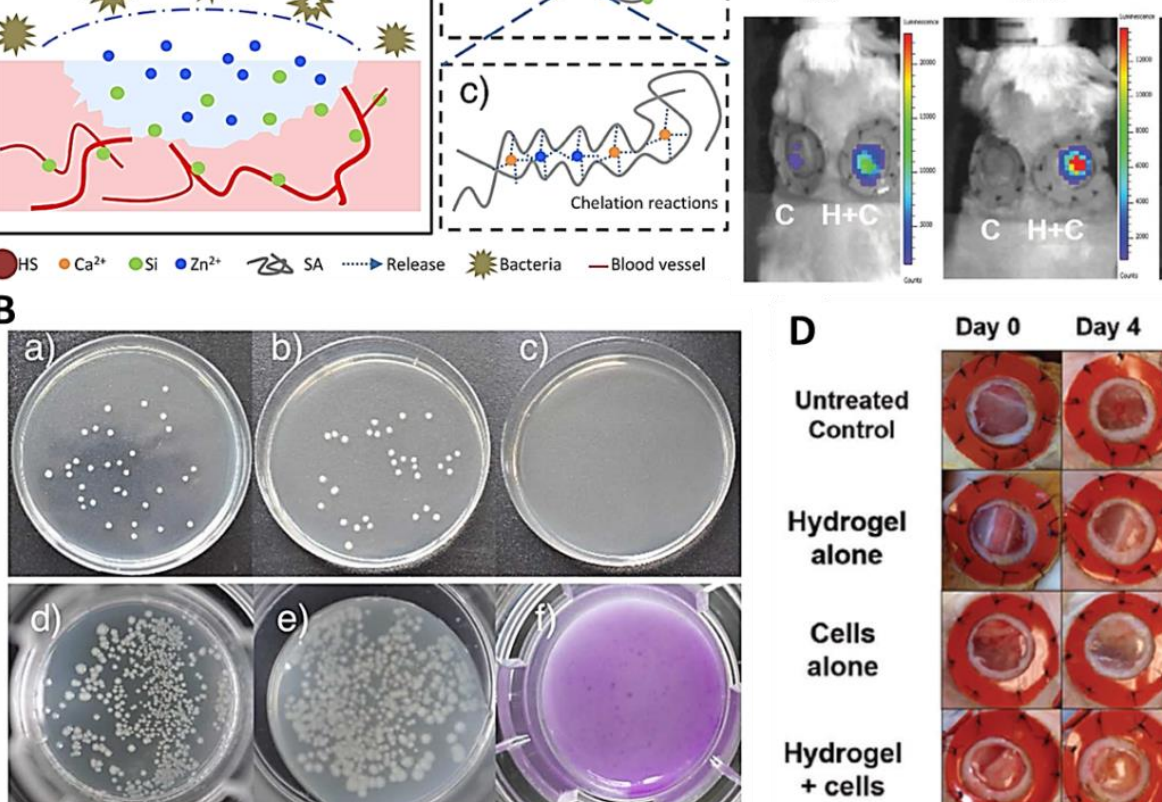

D12

D14
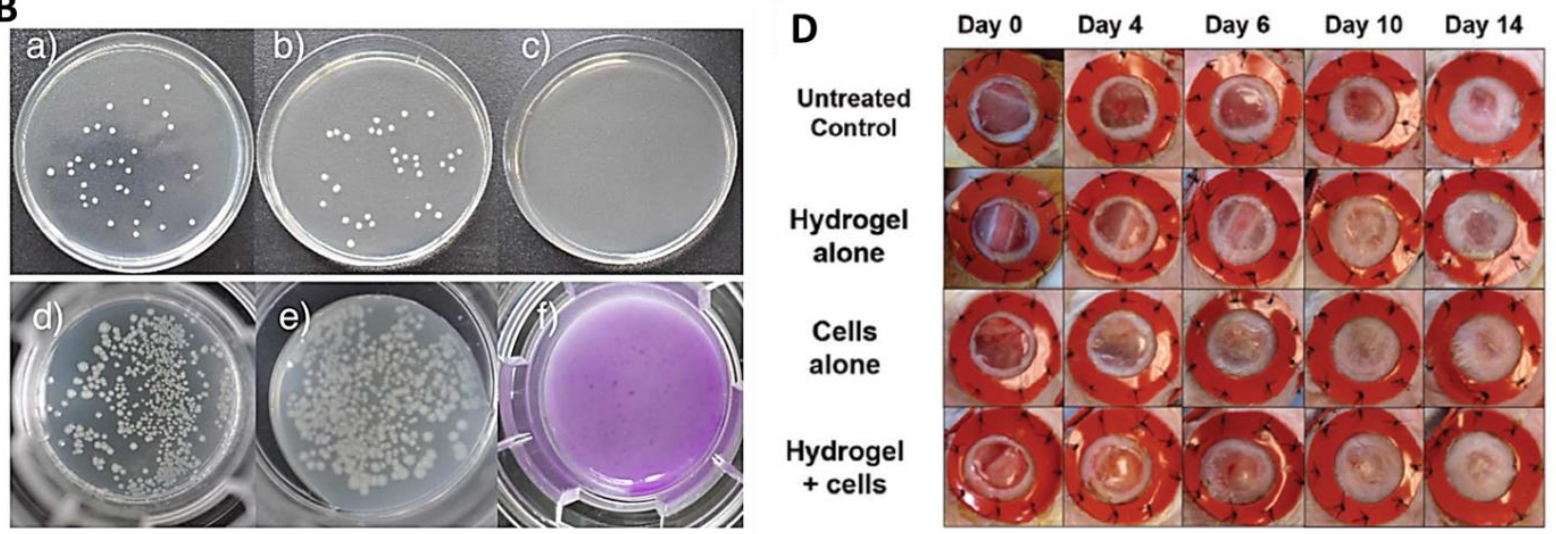


\section{WILEY-VCH}

Figure 8. A) Schematic illustration of the design and principles of SA/HS composite hydrogel for wound healing. In this structure, $\mathrm{Ca}^{2+}, \mathrm{Zn}^{2+}$ ions were able to act as crosslinkers for hydrogel formation through double ion cross-linking. Likewise, $\mathrm{Zn}^{2+}$ ions could act as antibacterial agents. a) Schematic representation of wound healing with hydrogel; b) The release of ions from HS particles into solution; c) structure of SA/HS composite hydrogel. B) Antibacterial effects of the SA/HS composite hydrogel against c E. coli. Upper: normal plate-counting method: a) control group; b) pure SA hydrogel group; c) SA/HS composite hydrogel group. Down: Contact type plate-counting method: d) control group; e) SA nutrient hydrogel group; f) SA/HS composite nutrient hydrogel group. Reproduced with permission. ${ }^{[103]}$ Copyright 2017, American Chemical Society. C) Bioluminescence images showing in vivo retention time for luciferase + ASCs delivered with "hydrogel + cells" $(\mathrm{H}+\mathrm{C})$ and "cells alone" (C) at 14 days after injection. Loading of ASCs into the IHs markedly improved their retention time in the site of injury. D) The photographs of wounds at $0,4,6,10,14$ days post wounding. The greatest rate of wound closure was seen in the hydrogel + cells group. Reproduced with permission. ${ }^{[62]}$ Copyright 2017, Wiley-VCH.

\section{Multiple therapies for IH-mediated tissue regeneration}

Tissue engineering approaches are still suffering from insufficient cell migration, the loss of cell viability following single-cell suspension injections, stress responses of cells into twodimensional structures, and initiation of inflammatory responses due to slow biodegradation rate of scaffolds. ${ }^{[108]}$ In addition, high cost, time-consuming processes, ${ }^{[109]}$ and poor bio-mimicking have limited their clinical applications. ${ }^{[110]}$ Alternatively, the long-term tissue remodeling by IHs provides an ideal platform that is able to encapsulate several types of drug molecules or cells without loss of their bioactivity for multi-therapy purposes. ${ }^{[111]}$ In fact, the combination of tissue resemblance and drug delivery represents a valid approach for combination therapy of tissue damage by IHs. ${ }^{[112]}$

\subsection{Cartilage tissue engineering}

Different therapeutic agents and various cell types can be loaded into the IHs to synchronously promote cartilage tissue regeneration. For example, Sá-Lima et al. ${ }^{[113]}$ developed stimuliresponsive chitosan-starch IHs combined with encapsulated adipose-derived stromal cells to improve articular cartilage regeneration. The authors reported that the incorporation of starch into the IHs improved the degradation profile and highly promoted chondrogenic differentiation 


\section{WILEY-VCH}

of adipose-derived stromal cells for cartilage regeneration. ${ }^{[113]}$ In another work, poly $(\mathrm{N}$ isopropylacrylamide)-co-acrylic acid IH containing transforming growth factor $\beta 3$ (TGF $\beta 3$ ) and chondrocytes were used for neocartilage formation. The engineered IHs showed higher chondrogenic differentiation at 8 weeks after injection compared to control IHs. ${ }^{[114]}$ Arora et al. ${ }^{[115]}$ also showed the suitable combination of cells, growth factors, and biomaterial scaffolds for articular cartilage defects. They developed an IH consisting of carboxymethyl cellulose (CMC), sulfated carboxymethyl cellulose (sCMC), and gelatin by in-situ crosslinking using HRP enzyme to deliver the transforming growth factor $\beta 1$ (TGF- $\beta 1$ ), the fat pad derived MSCs, and articular chondrocytes (ACs) to a cartilage defect area to induce TGF- $\beta 1$ mediated chondrogenesis. It was shown that using TGF loaded hydrogels caused better cell survival, higher sulfated glycosaminoglycans (sGAGs), and collagen deposition compared to control hydrogels. Moreover, loading TGF- $\beta 1$ into the hydrogels increased the expression and deposition of hyaline cartilage markers and, meanwhile, decreased the deposition of fibrocartilage and hypertrophy markers. Collectively, the authors concluded that cellulose-based hydrogels can be applied as cost-effective delivery systems for cartilage tissue engineering. ${ }^{[115]}$

\subsection{Cardiac tissue engineering}

Although several research groups have shown that conventional tissue engineering strategies (direct injection of various cell types into the heart tissue) might improve cardiac function following myocardial infarction (MI), a low rate of cell survival after transplantation have been reported. ${ }^{[116]}$ The combination of cell therapy and in-situ tissue-engineering approach can be considered as an ideal solution to protect the cell-graft from inflammation in the ischemic environment of the infarction and result in better functional outcomes than the direct injection of 


\section{WILEY-VCH}

human embryonic stem cell-derived cardiomyocytes into the infarcted region. ${ }^{[117]}$ For example, Song et al. ${ }^{[18]}$ developed acrylated HA IHs containing stem cell homing factor (SDF-1) and angiogenic peptides (Ac-SDKP) to simultaneously promote the recruitment of stem cells to the site of injury and increase the expression of angiogenic genes in a chronic heart failure model in rats. It was shown that IHs with single SDF-1 or Ac-SDKP failed to improve cardiac regeneration while combination therapy by IHs containing both factors resulted in enhancing the matured vessel formation and improving cardiac function. ${ }^{[18]}$ Nevertheless, advances in cellbased therapy of MI has still some disadvantages, including the low efficiency of cell delivery, low retention of cells in the site of injury, and poor survival of retained cells in the injury site that is a ROS rich microenvironment. To overcome these limitations, Hao et al. ${ }^{[19]}$ employed IHs prepared by a mild gelation process and by using ionic cross-linking between alginate and divalent cations $\left(\mathrm{Ca}^{2+}\right)$ in the presence of fullerenol nanoparticles to deliver stem cells into the site of injury in a rat model of MI. The hydrogel was prepared by a mild gelation process and by using ionic cross-linking between alginate and divalent cations $\left(\mathrm{Ca}^{2+}\right)$ in the presence of fullerenol nanoparticles (Figure 9A and 9B). The presence of fullerenol nanoparticles endows the hydrogel with antioxidant ability to capture the ROS free radicals. Also, the hydrogel could supply the structural support for the left ventricle. This hydrogel had no toxic effect on the brown adipose derived stem cells (BADSCs) and reduced the oxidative stress. It stimulated angiogenesis and led to increased survival, the cardiomyogenic differentiation (Figure 9C and 9D) and proliferation of BADSCs even under ROS microenvironment through the extracellular signal-regulated kinase (ERK) and p38 pathways activation and the c-Jun N-terminal kinase (JNK) pathway inhibition. ${ }^{[19]}$ 


\section{WILEY-VCH}

The lack of self-healing ability and conductivity are major limitations of injectable hydrogels for heart regeneration. ${ }^{[120]}$ These shortcomings result in the damage of hydrogels by external forces and hamper their prolonged application with a stable function for cell repair. In fact, owing to the contraction behavior of the heart, the cell delivery vehicles must tolerate a noticeable mechanical load following injection, which in turn increases the risk of damage to the IHs. ${ }^{[121]}$ Using conductive polymers within self-healable IHs can contribute to improving the proliferation and differentiation of the electrical responsive cells, such as cardiac and myoblast cells. ${ }^{[14,}{ }^{122]}$ For example, Dong et al. ${ }^{[123]}$ prepared conductive IHs with self-healing property based on the dynamic covalent Schiff base reaction between benzaldehyde moieties of dibenzaldehydeterminated PEG and amine moieties of chitosan-graft-aniline tetramer. The authors reported that the conductivity of IHs was very close to the native myocardium (a range from $2.29 \times 10^{-3}$ to $2.42 \times 10^{-3} \mathrm{~S} \mathrm{~cm}^{-1}$ ), mainly attributed to the presence of aniline tetramer in the structure of IHs. This IH indicated excellent properties as a cell delivery carrier that supported cell viability. ${ }^{[123]}$

\subsection{Tissue engineering of bone-associated defects}

One of the major disadvantages in bone tissue engineering is poor angiogenesis within tissueengineered grafts that might limit the clinical translation of this approach for the repair of large bone defects. ${ }^{[124]}$ To overcome this drawback, the potential of electrospun poly(lactic acid) (PLA) fiber-based membranes containing calcium phosphate $(\mathrm{CaP})$ ormoglass particles was evaluated by Oliviera et al. ${ }^{[125]}$ to increase angiogenesis. An IH containing $\mathrm{CaP}$ ormoglasses was prepared by dispersing the $\mathrm{CaP}$ ormoglasses within (hydroxypropyl) methylcellulose (HPMC) matrix. This composite had the ability to regulate the calcium release in a stable and sustained manner that led to the slower and longer healing of the inner part of the bone tissue. The 


\section{WILEY-VCH}

secretion of calcium ions by the recruitment of the bone marrow progenitor cells and sustained expression of proangiogenic cytokines contributed to the fast bone formation and promoted the angiogenesis. Nevertheless, proper bone regeneration by IHs needs a formulation with desirable self-healing and antibacterial properties. To address these requirements, the bone morphogenetic protein 2 (BMP-2) loaded adhesive liposomes (A-LIP) were incorporated into PEG hydrogel to prepare adhesive lipo-hydrogel (A-LIP-PEG) for promoting bone reconstruction. The hydrogel was prepared by coordination cross-linking between thiolated PEG (SH-PEG) and Ag in the presence of A-LIP. The coordination cross-linking between SH-PEG and Ag endowed the hydrogel with self-healing and injectability. Moreover, the presence of $\mathrm{Ag}$ in the formulation led to antibacterial activity against $E$. coli and $S$. aureus. After injection of the formulation into the target site, A-LIP was released from hydrogel and adhered to the tissue that created a longlasting and effective local drug depot. As a result, A-LIP-PEG with tissue adhesion ability showed more in vitro osteogenic differentiation and better bone reconstruction compared to common lipo-hydrogel (LIP-PEG). So that, the A-LIP-PEG group showed higher calcium nodules after 14 and 21 days of incubation in osteoblastic induction medium compared to LIPPEG and control groups (Figure 9E). This system showed good potential for the repair of the local damaged bone cavity. ${ }^{[126]}$ However, the A-LIP released by A-LIPPEG markedly increased the relative fluorescence intensity $(100 \pm 20 \%)$ as an indicator of osteocalcin $(\mathrm{OCN})$ expression (green), whereas LIP-PEG and control groups exhibited very weaker fluorescence intensity (46 \pm $8 \%$, and $4 \pm 1 \%$, respectively), indicating high potential of A-LIPPEG for enhancement of the osteogenic differentiation of BMSCs (Figure 9F). The assessment of bone healing by micro-CT scans demonstrated that mineralization and the healing of the fracture in the A-LIP-PEG treatment group were greater than the control and LIP-PEG groups (Figure 9G). 


\section{WILEY-VCH}
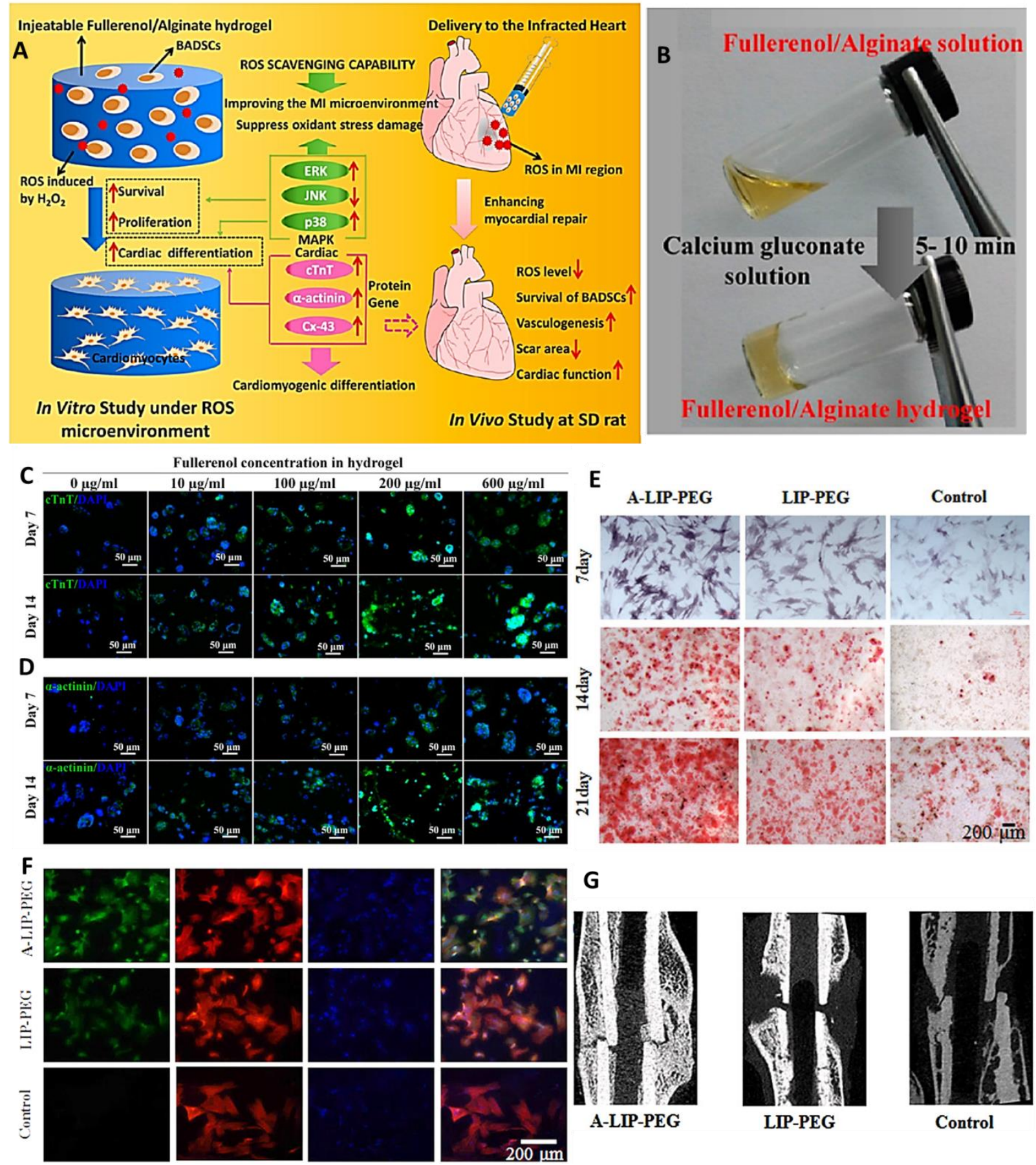

Figure 9. A) Schematic illustrations of the fullerenol/alginate hydrogel effects on the survival, proliferation, and differentiation of BADSCs in vitro and positive effects on cardiac repair in vivo. B) Fullerenol/alginate hydrogel synthesis process and its transition from sol to gel within 5-10 min following the addition of calcium gluconate solution. C) Effect of fullerenol/alginate hydrogel on cardiomyogenic differentiation of BADSCs under oxidative stress. Immunofluorescence staining of cTnT marker at days 7 and 14 in the presence of $\mathrm{H}_{2} \mathrm{O}_{2}$. There was a direct association between fullerenol content and the expression of cTnT marker at days 7 and 14 in the presence of $\mathrm{H}_{2} \mathrm{O}_{2}$. D) Effect of fullerenol/alginate hydrogel on cardiomyogenic differentiation of BADSCs under oxidative stress. Immunofluorescence 


\section{WILEY-VCH}

staining of the $\alpha$-actinin marker at days 7 and 14 in the presence of $\mathrm{H}_{2} \mathrm{O}_{2}$. There was a direct association between fullerenol content and the expression of the $\alpha$-actinin marker at days 7 and 14 in the presence of $\mathrm{H}_{2} \mathrm{O}_{2}$. Reproduced with permission. ${ }^{[119]}$ Copyright 2017, American Chemical Society. E) Upper part: The ALP-stained BMSC cells in the normal medium as the control; middle and lower part: both A-LIP-PEG and LIP-PEG in the osteoblastic induction medium after 7 days of incubation. Alizarin red-stained (dark red) images of the BMSC cells in the normal medium as the control, and Alizarin red-stained photographs of both A-LIP-PEG and LIP-PEG in the osteoblastic induction medium after 14 and 21 days of incubation. F) The amount of OCN protein expression in control, A-LIP-PEG, and LIP-PEG groups in the osteoblastic induction medium at the end of the 21st day of osteogenic differentiation. A-LIP-PEG group markedly increased the expression of OCN protein as an early marker of in vitro osteogenic differentiation compared to control and LIP-PEG groups. G) Assessment of in vivo bone callus formation at 8 weeks post-treatment using Micro-CT scans. The highest amount of in vivo bone callus formation was seen in the A-LIP-PEG group. Reproduced with permission. ${ }^{[126]}$ Copyright 2019, Nature Publishing Group.

In another interesting example, Kocak et al. ${ }^{[21]}$ developed $\mathrm{pH}$ and thermosensitive IHs based on CS and hydroxyapatite (HA) composite materials loaded with heparin (Hep) to improve angiogenesis towards repair and regeneration of bone (Figure 10A). Hydrogel precursor solutions with various concentrations of Hep were prepared using sol-gel technique. The researchers used an ex-ovo chick chorioallantoic membrane (CAM) assay to evaluate angiogenesis by applying injectable solutions on CAM. Histological analyses confirmed inducing microvascularity in CAM assay. Likewise, incorporation of lower concentrations of Hep in the IHs resulted in more efficiency in pro-angiogenic response (Figure 10B). The total survival rates of CAM experiments before and after sample implantation were between $45-50 \%$ and $70-80 \%$, respectively. ${ }^{[21]}$ 


\section{WILEY-VCH}

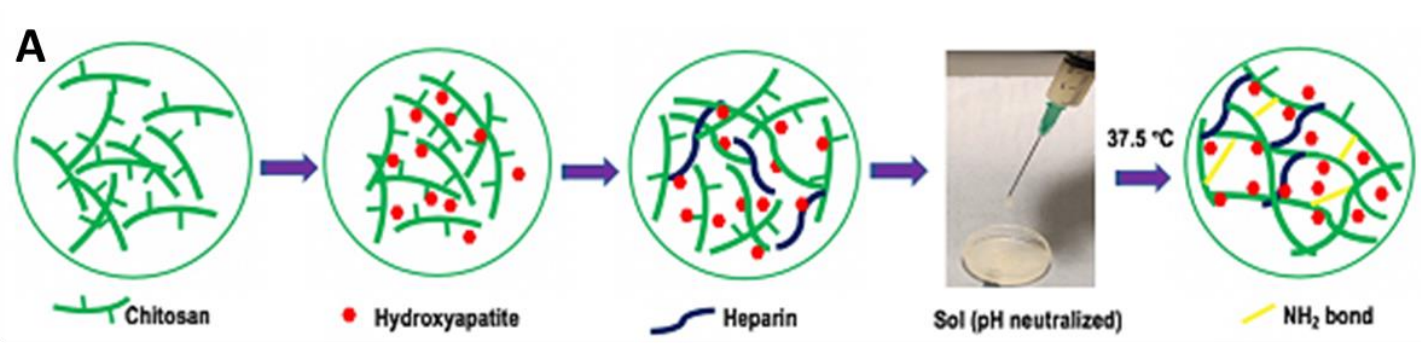

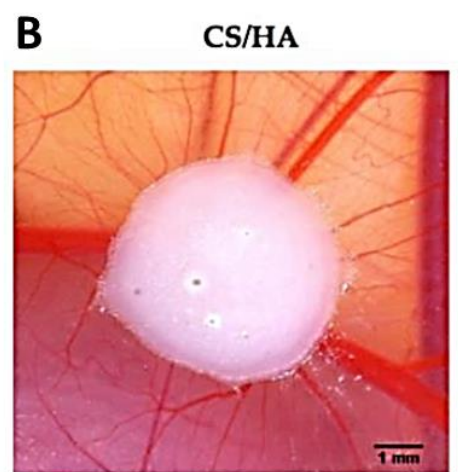

(a)

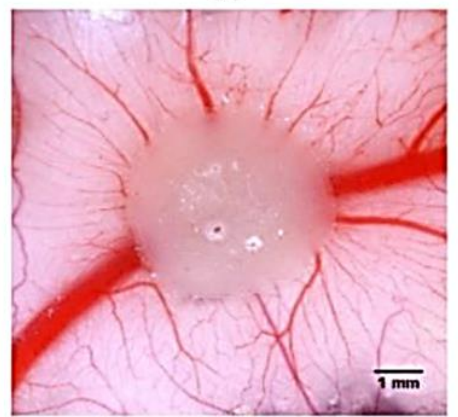

(d)

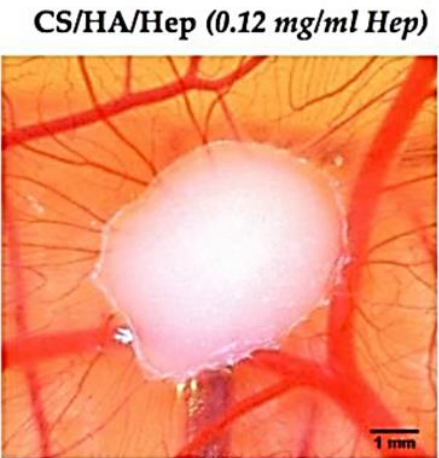

(b)

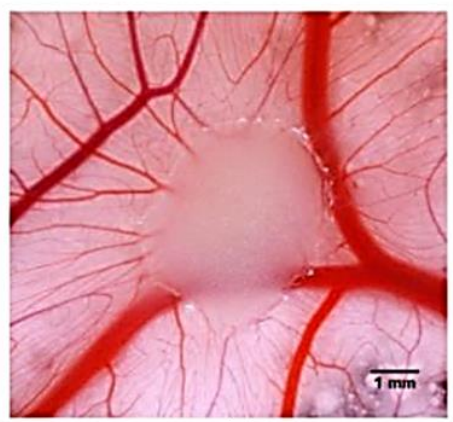

(e)

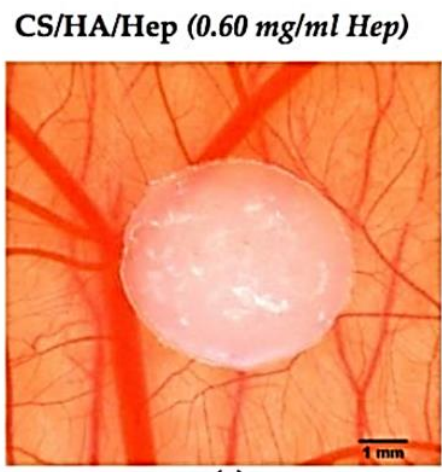

(c)

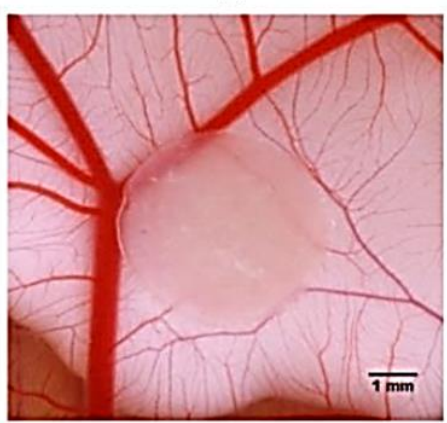

(f)

Figure 10. A) Schematic illustrations of preparation of CS/HA/Hep IHs. B) CAM Images at day 14 of embryo incubation for examination of angiogenic response of IHs. (a) CS/HA; (b) CS/HA/Hep (0.12 $\mathrm{mg} / \mathrm{mL} \mathrm{Hep}$ ); (c) $\mathrm{CS} / \mathrm{HA} / \mathrm{Hep}(0.60 \mathrm{mg} / \mathrm{mL} \mathrm{Hep})$, and images after white cream injection underneath CAM to make blood contrast: (d) CS/HA; (e) CS/HA/Hep $(0.12 \mathrm{mg} / \mathrm{mL} \mathrm{Hep})$; (f) $\mathrm{CS} / \mathrm{HA} / \mathrm{Hep}(0.60$ $\mathrm{mg} / \mathrm{mL}$ Hep). Reproduced with permission. ${ }^{[21]}$ Copyright 2020, MDPI.

For the treatment of rheumatoid arthritis (RA) as a disease with complex pathogenicity, the treatment systems should be acted through multiple mechanisms around anti-inflammation effects and osteogenesis, while traditional methods suffer from side effects and also they were limited to one or two mechanisms and ignored the other mechanisms. In an exploration by Pan et al., ${ }^{[127]}$ a new platform composed of black phosphorus nanosheets (BPNs) into platelet-rich 


\section{WILEY-VCH}

plasma (PRP)-CS thermoresponsive IH was employed for RA treatment (Figure 11A). BPNs with photothermal conversion efficiency (Figure 11B) and generation of ROS under NIR irradiation could remove hyperplastic synovial tissues. Moreover, PRP could facilitate adhesion and proliferation of mesenchymal stem cells (MSCs) along with good mechanical properties and biodegradability of CS hydrogel (Figure 11C). Also, CS thermosensitive hydrogel with excellent rheological property, in addition to the lubrication and cartilage protection, by thermosensitivity and biodegradability could control the drug release. In addition, the degradation products of BPNs could be in-situ converted to P-based agents that, through calcium-extracted biomineralization by the in-situ phosphorus-driven of the BPNs, led to improving the osteogenesis process. Both in vitro and in vivo experiments confirmed that the BPNs/CS/PRP thermoresponsive hydrogel had good potential in the treatment of RA, and the hydrogel with a multifaceted mechanism of action paves the way for curing this complex disease. ${ }^{[127]}$

In another example, Parameswaran-Thankam et al. ${ }^{[128]}$ prepared injectable thermoresponsive hydroxypropyl guar-graft-poly(N-vinyl caprolactam) (HPG-g-PNVCL) copolymer using graft polymerization method and then modified it with nano-hydroxyapatite (n-HA) by in situ covalent cross-linking using divinyl sulfone (DVS) cross-linker to create HPG-g-PNVCL/n-HA/DVS structure composite. The hydroxypropyl guar gum as a plant-derived polymer was used to improve the bioactivity of poly( $\mathrm{N}$-vinyl caprolactam) and hinder the initiation of acute inflammatory response. In order to improve antibacterial activity, the researchers loaded ciprofloxacin, a commonly known antibiotic, into the composite. In vitro experiments revealed that the hydrogel was capable of releasing the drug molecules in a sustained manner. In vitro biomineralization study via immersion in simulated body fluid (SBF) for 14 days displayed that the apatite like structure was deposited on the surface of HPG-g-PNVCL/ n-HA/DVS scaffold 


\section{WILEY-VCH}

that confirmed by SEM and in the presence of $\mathrm{n}-\mathrm{HA}$, the $\mathrm{Ca} / \mathrm{P}$ ratio increased from 0.83 to 2.00 within 7 days that is similar to the human body (Figure 11D). As a result, this system with the calcium-rich apatite-forming ability, great bioactivity, and providing an excellent induction of osteoblast cell differentiation, was very effective for tissue regeneration. ${ }^{[128]}$

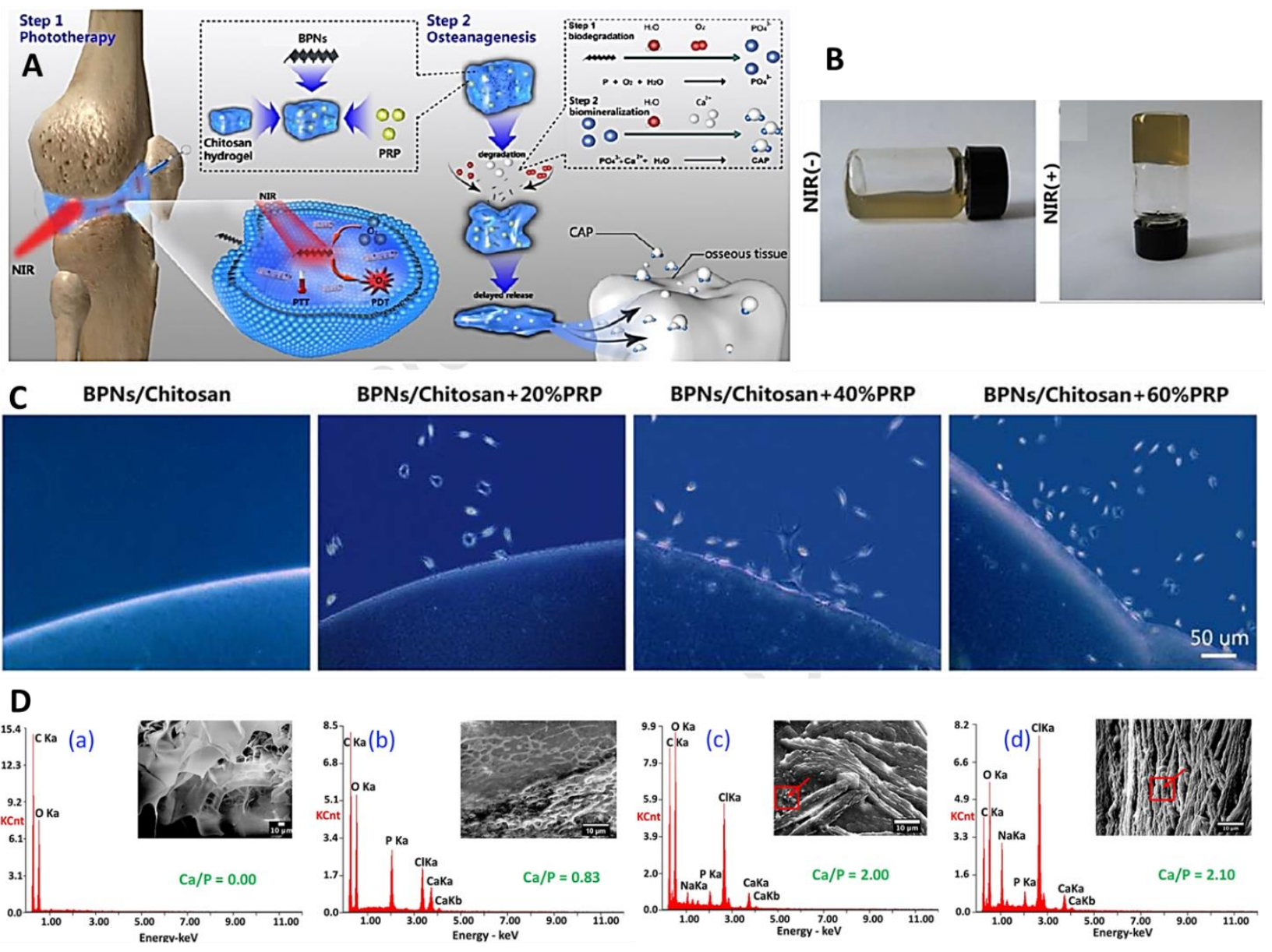

Figure 11. A) Schematic illustration of the mechanism of BPNs/CS/PRP thermoresponsive hydrogel for the treatment of RA. B) Representative image of the thermoresponsive property of BPNs/CS/PRP in the absence and presence of NIR. C) Inverted microscope images showing the effect of various content of PRP $(0,20,40$, and 60) on the adhesion of MSCs. There was a direct association between the content of PRP and cell adhesion. Reproduced with permission. ${ }^{[127]}$ Copyright 2020, Elsevier. D) In vitro biomineralization of (a) HPG-g-PNVCL without n-HA, (b) HPG-g-PNVCL/n-HA/DVS on day 0, and HPG-g-PNVCL/n-HA/DVS after (c) 7 and (d) 14 days. Reproduced with permission. ${ }^{[128]}$ Copyright 2018, American Chemical Society.

\subsection{Dental tissue engineering}




\section{WILEY-VCH}

Dental disorders, such as periodontal disease, tooth lesion and loss strongly affect masticatory efficiency and facial aesthetics. Combined therapy by injectable biomaterials is a valid approach to accelerate dental regeneration. ${ }^{[129]}$ For example, Xu et al. ${ }^{[130]}$ developed thermosensitive IH based on CS, b-sodium glycerophosphate (b-GP), and gelatin to accelerate periodontal regeneration by controlled release of erythropoietin (EPO) and aspirin (Figure 12A). A continuous release of aspirin and EPO from IHs was found without any toxicity both in vitro and in vivo, for at least 21 days. Micro-CT analyses and immunohistochemistry staining showed that CS/b-GP/gelatin IHs could terminate the inflammation and recover the height of the alveolar bone through down-regulation of cyclooxygenase-2 (COX-2) and matrix metalloproteinase-9 (MMP-9) proteins (Figure 12B). ${ }^{[130]}$

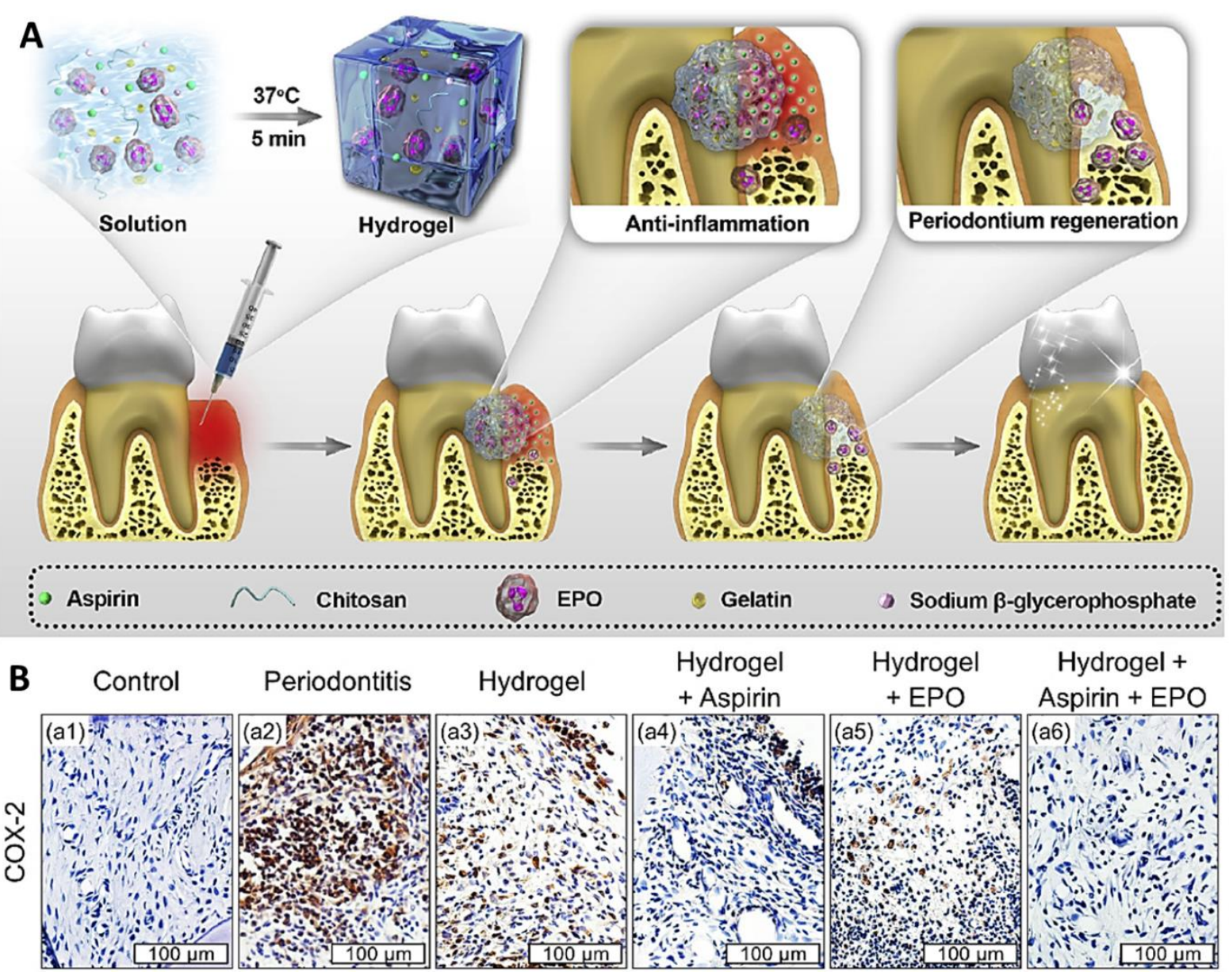




\section{WILEY-VCH}

Figure 12. A) Schematic illustration of thermoresponsive IH fabricated from chitosan/sodiumglycerophosphate/gelatine for controlled release of EPO and aspirin towards accelerating periodontal regeneration. B) ) IHC staining of COX-2 two weeks after implantation. (a1) Control, (a2) Periodontitis, (a3) Hydrogel, (a4) Hydrogel + Aspirin, (a5) Hydrogel + EPO, (a6) Hydrogel + Aspirin + EPO. Brown color shows the expression of COX-2 as an inflammation marker. Reproduced with permission. ${ }^{[130]}$ Copyright 2019, Elsevier Ltd.

In another study, Tan et al. ${ }^{[131]}$ prepared a biocompatible supramolecular hydrogel of SDF1/BMP-2/NapFFY through co-assembly of hydrogelator NapFFY with BMP-2 and SDF-1 for the regeneration of periodontal tissues. The small $\mathrm{G}^{\prime}$ and $\mathrm{G}^{\prime \prime}$ values of hydrogels (about 1 to 30 $\mathrm{Pa}$ ) demonstrated that they were injectable and had the ability to fill up the irregular periodontal bone defects. They found that SDF-1 and BMP-2 loaded hydrogel was filled in the defect area of tissue, and sustained release of stimulators of periodontal tissue reconstruction (SDF-1 and BMP-2) resulted in the initiation of the periodontal bone regeneration process (Figure $13 \mathrm{~A}$ and

13B). ${ }^{[131]}$ Nevertheless, poor ability of revascularization, particularly in endodontic regenerative therapies, due to the complicated anatomy of the root canal system and the narrow apical access, is a big challenge in the field of tissue engineering. In a study by Silva et al., ${ }^{[132]}$ injectable and viscoelastic HA-based hydrogels were employed for releasing the chemotactic and angiogenic growth factors (GFs). HA hydrogels were enriched with cellulose nanocrystals (CNCs) and platelet lysate (PL). The CNC endowed the HA hydrogel with mechanical performance and stability against enzymatic degradation. Moreover, PL provided chemotactic and proangiogenic GFs (platelet-derived growth factor (PDGF), VEGF). These IHs provided the controlled release of chemotactic and pro-angiogenic GFs (PDGF and VEGF). The hydrogel, due to the PL content, increased the viability of the encapsulated human dental pulp cells (hDPCs). Also, the released GFs from hydrogel recruited dental pulp-origin cells in an in vitro and improved cell sprouting in an ex vivo model. This system, with a great capability of simultaneously acting as a 


\section{WILEY-VCH}

GFs controlled delivery system and as a supportive matrix for cell culture, recruitment, and revascularization induction, showed a great potential for vascularized tissue engineering applications, such as in regenerative dentistry. ${ }^{[132]}$
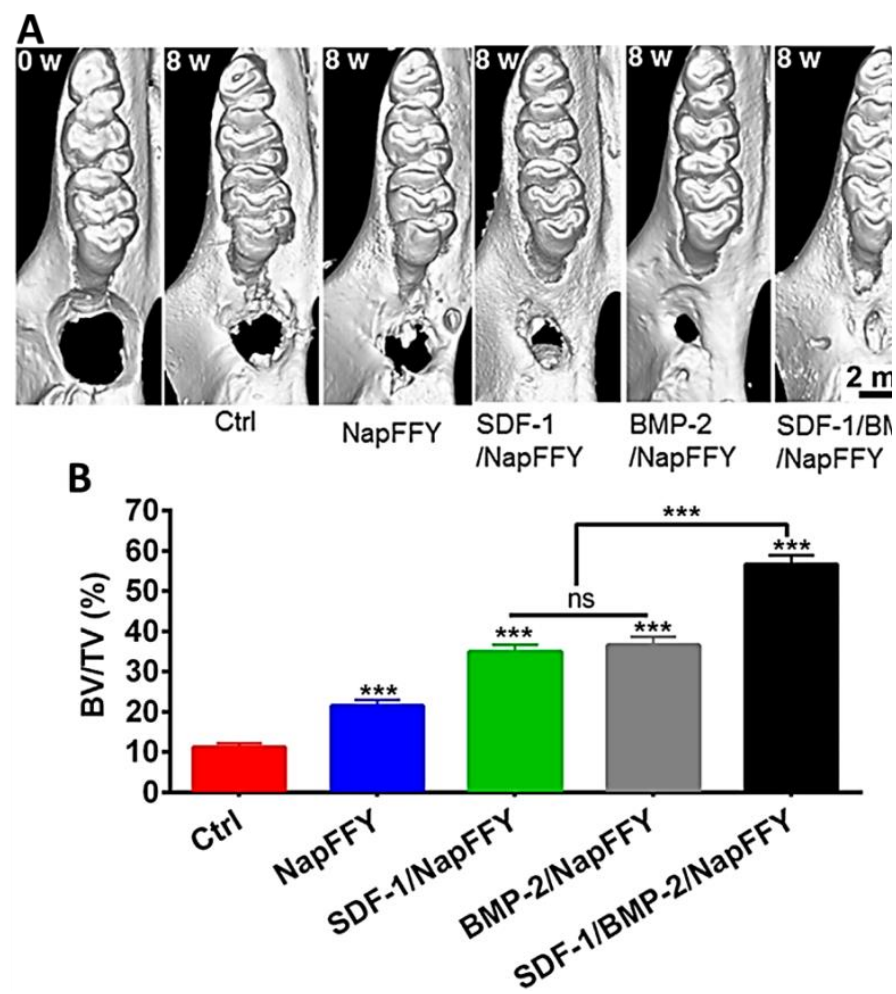

Figure 13. A) Micro-CT images of the periodontal bone defect regions in the maxillae of Sprague Dawley rats of various groups (bone defect sizes $=20 \mathrm{~mm}^{3}$ ). B) Bone volume fractions of defect regions in various experimental groups 8 weeks after defect. Data are presented as mean \pm SD $(n=3)$. ***p < $0.001 \mathrm{vs}$. control. Not significant (ns). Scale bar $=2 \mathrm{~mm}$. Reproduced with permission. ${ }^{[131]}$ Copyright 2019 American Chemical Society.

\subsection{Nerve tissue engineering}

The IHs can be used as carriers for neural stem cells and growth factors to repair nerve damage. ${ }^{[133]}$ For example, Zhao et al. ${ }^{[134]}$ developed a thermosensitive IH containing nerve growth factor (NGF) and heparin-poloxamer (HP) for spinal cord injury (SCI) regeneration. They firstly synthesized HP based on EDC/NHS method and then prepared NGF-HP in situ hydrogels using cold method. NGF-HP hydrogels exhibited good bioactivity. In vivo 


\section{WILEY-VCH}

experiments in a rat model of SCI showed that NGF-HP hydrogels exerted higher improvement in neuron functions and tissue morphology as well as higher inhibition on the formation of glial scars than free HP hydrogel and NGF treatment groups. The synergistic effects of NGF-HP hydrogels on SCI regeneration could be attributed to neuroprotective effects of NGF and antiinflammatory effects of heparin. ${ }^{[134]}$ In 2017, the same group deigned glial cell-derived neurotrophic factor (GDNF)-HP hydrogels using cold method and found that their injection into the lesion epicentre after SCI had stronger effects on neural stem cell proliferation and axonal regeneration compared to free HP hydrogel and GDNF treatment groups. ${ }^{[135]} \mathrm{Li}$ et al. ${ }^{[136]}$ loaded bFGF and NGF into the thermosensitive HP hydrogels for peripheral nerve regeneration in diabetic rats. The HP hydrogels had the capacity to load large amounts of growth factors (GFs) and release them in a controlled manner. Injection of GFs-HP hydrogels in the site of injury resulted in a more effective proliferation of schwann cells, axonal regeneration and remyelination than HP hydrogel alone or direct GFs administration. ${ }^{[136]}$

IHs can also be applied to regenerate damaged nerves following ischemic stroke through simultaneous delivery of stem cells and growth factors. For instance, Moshayedi et al. ${ }^{[137]}$ deigned a modified HA hydrogel with heparin bound growth factors and MMP degradable motifs to synchronously deliver human neural progenitor cells (iPS-NPCs) and growth factors for nerve regeneration following ischemic stroke. In their study, growth factors and heparin were mixed with the gel precursor solution before the addition of a MMP-degradable peptide crosslinker. Results have shown that cells encapsulation into the IHs had a significant effect on increased astrocytic differentiation of iPS-NPCs within the stroke cavity through up-regulation of GFAP and S100b markers. ${ }^{[137]}$ 


\section{WILEY-VCH}

\section{Other applications of IHs for combined therapy}

Regarding the fascinating properties and capabilities of IHs, the researchers have also assessed their performance in the co-delivery of therapeutics to provide multiple therapies. In this respect, Tuladhar et al. ${ }^{[23]}$ concentrated on co-delivering therapeutics to the brain, which is a hard task due to the blood-brain barrier (BBB) and the off-target influences induced by the systemic delivery. The drugs, including cyclosporine A (CsA) and erythropoietin (EPO) can result in detrimental side effects; however, the local delivery of them could be a promising route to reduce the side effects. So, they synthesized a type of IH to circumvent the BBB and accordingly codelivered the CsA and EPO controllably and locally for the first time. They loaded the drugs into poly (lactide-co-glycolide) (PLGA) particles, then dispersed them in hyaluronan and methylcellulose (HAMC) gel to form a modular delivery system. In order to limit any drug leakage to the cerebrospinal fluid (CSF), they enclosed the composite in a polycarbonate casing and sealed it with dental cement, and in order to limit the tissue damage from intracranial implants, the composite was implanted onto the surface of the rat brain, four days after injury. As reported, both drugs could diffuse into the sub-cortical neural stem and progenitor cell (NSPC) niche and could be retained there for about 32 days. It was found that the CsA improved the plasticity in the striatum, and the EPO was effective in stimulating the endogenous NSPCs. Moreover, these effects were viable only through the local and sustained co-delivery of these drugs, which finally enhanced the tissue repair, so that, in the CsA + EPO treated group, the lesion volume significantly was decreased at day 46 compared to day 4 ( $\mathrm{p}<0.05)$ (Figure 14A). But, this significant difference was not observed in single drug-treated groups. In CsA treated group, the lesion volume was decreased near $23 \%$, while this value was $27 \%$ for EPO-alone. Since the lesion volume was decreased by 50\% in CsA + EPO treated group (Figure 14B), this 


\section{WILEY-VCH}

issue indicated that CsA and EPO had an additive effect and independent mechanisms on stroke lesion. $^{[23]}$

It has been reported that sequestering or attachment of pro-angiogenic growth factors to nanoparticles and incorporation into the IHs cause sustained activation of cell-surface receptors and higher efficiency for the treatment of killing diseases in comparison to exogenous (unbound) delivery of growth factors. ${ }^{[138]} \mathrm{Nih}$ et al. ${ }^{[139]}$ developed in situ gelling HA hydrogels for codelivery of nanoparticle-clustered VEGF and heparin nanoparticles to improve brain repair after stroke. They found that heparin nanoparticle-clustered VEGF had stronger effects on normal vascular development, reducing microglia activation, promoting axonal ingrowth and migration of immature neurons from the subventricular zone to the infarcted region compared to traditional delivery of VEGF in the brain. It was shown that anti-inflammatory properties of naked heparin particles could counterbalance the inflammatory effect of VEGF and contribute to the formation of a pro-repair environment in the infarcted region. ${ }^{[139]}$

In addition, Boffito et al. ${ }^{[140]}$ developed an IH for co-delivery of copper ions and ibuprofen. To this end, at first, they synthesized the nano and microparticles of copper-substituted bioactive mesoporous glasses (Cu-MBGs), followed by ibuprofen loading via the incipient wetness technique. Then, this composite was dispersed into the amphiphilic polyurethane-based thermosensitive IH. As declared, the gelation behavior of this $\mathrm{IH}$ was not affected by the loaded drug and ions. The results revealed that this approach could provide the sustained and codelivery of the $\mathrm{Cu}^{+2}$ and ibuprofen, where the released copper ions stimulated the chemical degradation of polyurethane and could control the gel residence time at the pathological site.

Overall, this research verified that the $\mathrm{IH}$, along with the MBGs could be effectively engineered 


\section{WILEY-VCH}

for controllable and local co-delivery of therapeutic ions and drugs, which is highly demanded in the biomedical field. ${ }^{[140]}$
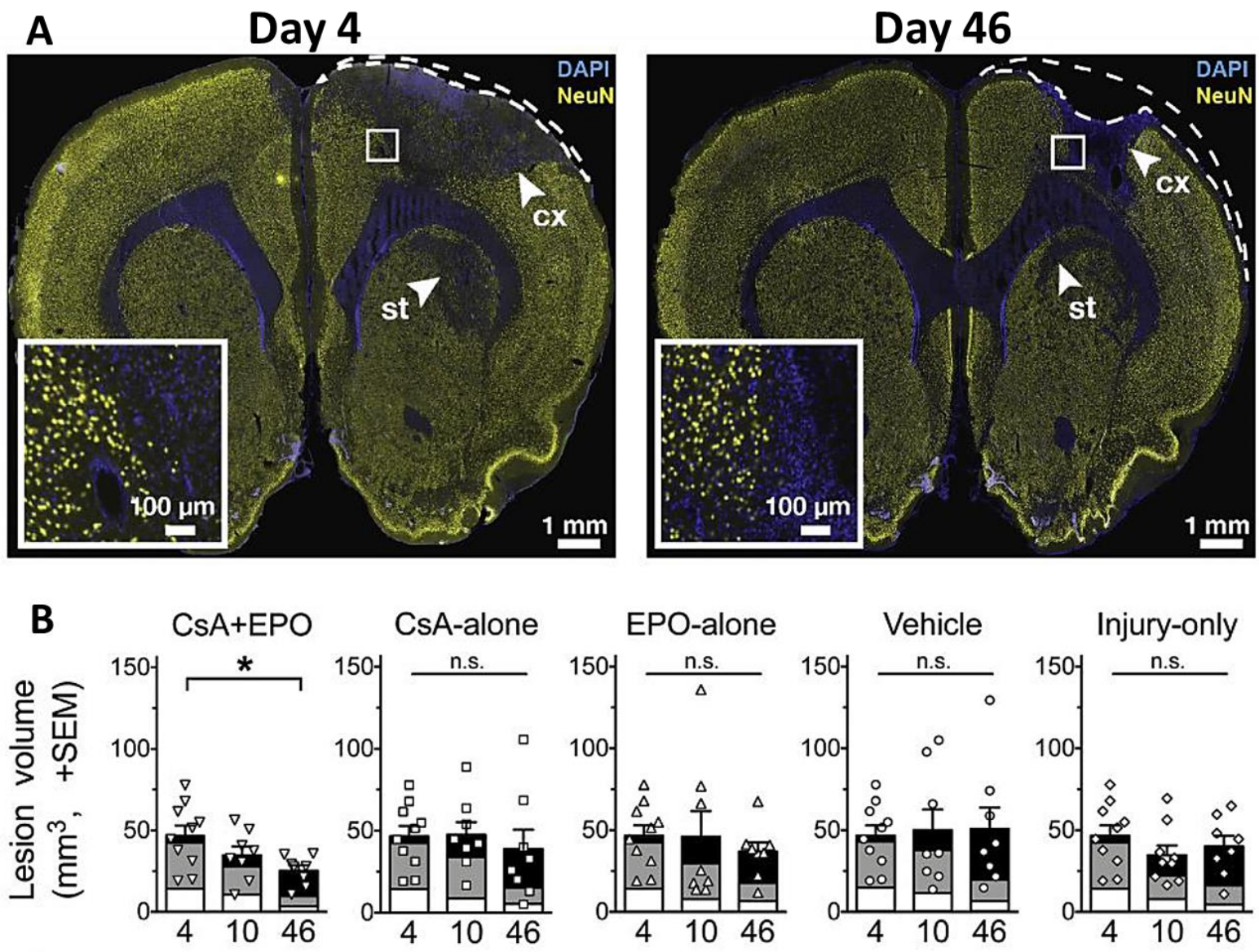

Cavity

Cortical Infarct

Striatal Infarct

\section{Days post-stroke}

Figure 14. A) Representative images of stroke-injured brains stained with NeuN for mature neurons, showing a shift in injury composition over time. The cavity (dashed outline) is determined by the loss of DAPI staining and the cortical and striatal infarcts (arrowheads) are determined by the loss of NeuN staining in comparison to the uninjured contralesional hemisphere. Calculation of the lesion volume was based on the following formula in both cx and st: the stroke cavity (DAPI volume) + stroke infarct (DAPI ${ }^{+} \mathrm{NeuN}^{-}$volume). B) Quantification of lesion volume before implant at day $4(\mathrm{n}=10$ rats) and after implant at day 10 (Injuryonly, $n=9$ rats; all other groups $n=8$ rats) and day 46 ( $n=8$ rats per group) poststroke, showing reduced stroke lesion volume with CsA + EPO treatment. Data are defined as mean + S.E.M. Significant differences were determined by a one-way ANOVA with Dunnett's posthoc test vs. day 4 (2 comparisons); * $p<0.05$. Reproduced with permission. ${ }^{[141]}$ Copyright 2020, Elsevier.

Fan et al. synthesized an IH from natural polysaccharides through Schiff's base reaction for codelivery of drugs and cell scaffolds. ${ }^{[142]}$ During the synthesis procedure, cross-liking of the IH 


\section{WILEY-VCH}

was carried out by CMC and oxidized chondroitin sulfate (OCS), where the mechanism was the reaction between amino and aldehyde groups. In terms of co-delivery by this $\mathrm{IH}$, bovine serum albumin (BSA) was loaded in the CS-based microspheres and embedded into the CMC-OCS IH. The CS microspheres could improve the mechanical and bioactive properties of the IH. Rufaihah et al. ${ }^{[143]}$ addressed acute MI through GF therapy by IHs. They synthesized an IH from the polyethylene glycol-fibrinogen (PF), which was employed for the controllable and co-delivery of VEGF and angiopoietin-1 (ANG-1) (named as PF-VEGF-ANG1) with the final aim of improving the myocardial repair and function. Both VEGF and ANG-1 were released controllably in a period of 30 days. Accordingly, the cardiac muscle was preserved due to the synergistic effects of VEGF and ANG-1. The 1\% PF-VEGF-ANG1 group exhibited the greatest capillary density in the infarct region and peri-infarct regions compared to the saline-treated group and the 1\% PF-treated group (Figure 15A). Moreover, the PF-VEGF-ANG-1 hydrogel could also act as mechanical support to attenuate adverse myocardial remodeling. Overall, the combined therapy via co-delivery of therapeutics through an IH could result in preventing postMI cardiac dysfunction. ${ }^{[143]}$

Zhang et al. ${ }^{[144]}$ focused on simultaneous delivery of drugs and 3D cell cultivation of L929 cells by an $\mathrm{IH}$, which was synthesized by mixing the glycol CS, and a dibenzaldehyde terminated copolymer poly(N-isopropyl acrylamide)-co-poly(acrylic acid) (DF poly(NIPAM-co-AA)) solutions and through dynamic imine bonds formation between benzaldehyde and amine groups that endowed the resultant hydrogel with self-healing and injectability property (Figure 15B). In this work, to study the application of the prepared IH in drug delivery, cisplatin as an anticancer drug and the rhodamine B were loaded as drug models. As stated by the authors, this IH was responsive to the $\mathrm{pH}$ and temperature and could release the drug controllably as a function of 


\section{WILEY-VCH}

surrounding $\mathrm{pH}$ and temperature. This non-cytotoxic IH was successfully applied for 3D cell cultivation of L929, so that the L929 cells showed good viability (i.e., 90\%) after incubating with hydrogel for 24 hours (Figure 15C) that indicated hydrogel by providing a 3D environment for cells to grow is a nice cell carrier candidate. This promising performance can be regarded for further works on multiple therapy by IHs in simultaneous drug delivery and cell therapy. ${ }^{[144]}$

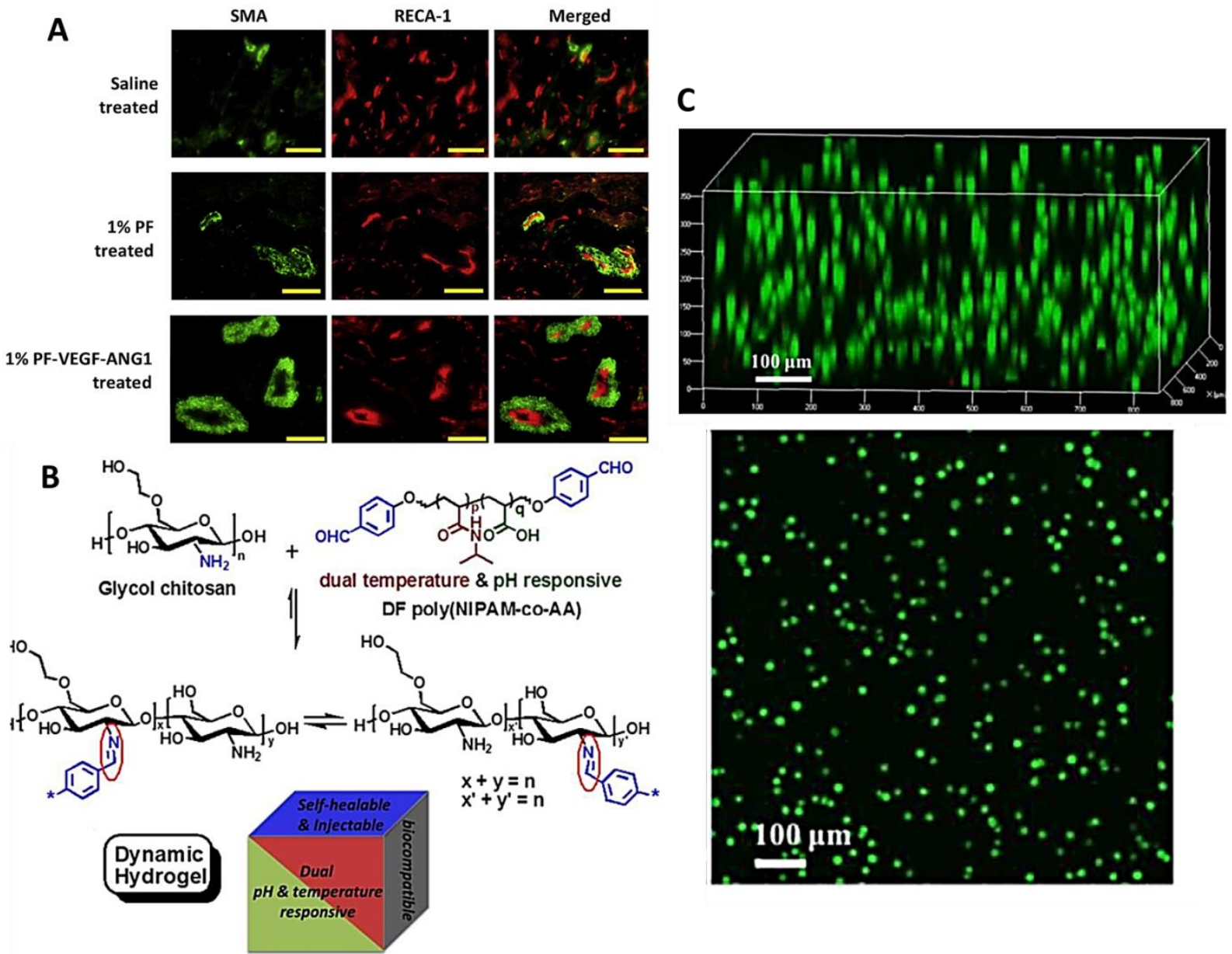

Figure 15. A) Immunofluorescence staining of blood vessels in the infarct regions showed the greatest capillary density with larger diameters in 1\% PF-VEGF-ANG1 group compared to the saline-treated group and the 1\% PF-treated group (Scale bar: $100 \mu \mathrm{m}$ ). Reproduced with permission. ${ }^{[143]}$ Copyright 2017, Elsevier. B) Preparation of the injectable, self-healable, and dual pH/temperature-responsive hydrogel using glycol CS and DF poly (NIPAM-co-AA). C) Confocal microscopy images (3D (left) and maximum z-axis projection (right) views) of viability for L929 cells encapsulated in the hydrogel after 24 h (viable cells: green, dead cells: red). Reproduced with permission. ${ }^{[144]}$ Copyright 2016, The Royal Society of Chemistry. 


\section{WILEY-VCH}

Pentlavalli et al. ${ }^{[145]}$ synthesized an in-situ forming biocompatible IH through the simple freeradical polymerization of $\mathrm{N}$-isopropyl acrylamide (NIPAAm), which was done on the alginate backbone while using the ammonium persulfate (APS) and $N, N, N^{\prime}, N^{\prime}-$ tetramethylethylenediamine (TEMED). The resultant hydrogel showed thermoresponsive behavior at LCST of $32{ }^{\circ} \mathrm{C}$ that is near to normal body temperature. The hydrogel showed biocompatibility and biodegradability, which was degraded by about $70 \%$ after eight weeks through surface degradation that is desirable for long-term drug delivery. Regarding the delivery behavior, it was found that the large protein molecule BSA, because of interaction with the polymeric network, was released slower compared to $\mathrm{NaF}$ from the hydrogel at the conditions of pH 7.4 and temperature of $37{ }^{\circ} \mathrm{C}$. Moreover, the alginate viscosity could adjust the mechanical and structural properties of the $\mathrm{P}(\mathrm{Alg}$-g-NIPAAm). This novel thermosensitive IH was also exploited in local and sustained co-delivery of human osteosarcoma (MG63) cells and porcine bone marrow-derived mesenchymal stem cells (pBMSCs). The pBMSCs with uniform distribution were encapsulated within the IH. By incubating in osteogenic and chondrogenic media, the osteogenic and chondrogenic potential of pBMSCs encapsulated in the P(Alg-gNIPAAm) hydrogel were evaluated, and after a two-week culture period in both osteogenic and chondrogenic conditions, viability was increased from an initial value of $60 \%$ to more than $80 \%$. The histological evaluation indicated intense collagen and sGAGs staining for hydrogels incubated in chondrogenic conditions compared to osteogenic conditions. At the same time, higher mineralization was observed for hydrogel incubated in osteogenic conditions. In addition, the intense DNA content of the hydrogel incubated in chondrogenic conditions was observed that was due to the proliferative impact of TGF- $\beta 3$ in its media. ${ }^{[145]}$ 


\section{WILEY-VCH}

Linh et al. ${ }^{[146]}$ developed an in-situ forming IH through in-situ enzymatic crosslinking between tyramine-conjugated gelatin and hydroxyl phenyl acetamide CS in the presence of HRP as catalyst and hydrogen peroxidase as an antioxidant to deliver human adipose-derived stem cells (hADSC) and platelet-derived growth factor (PDGF) simultaneously with the aim of vascularization. The in vitro experiment and subcutaneous hydrogel implantation into Balb/c nude female mice revealed good compatibility of the hADSC with hydrogel formulation. Histological analysis showed that the biocompatible scaffold with a unique branched network and channels could support cell infiltration and vascularization. ${ }^{[146]}$

\section{Conclusions and future perspectives}

The combined use of several therapeutic agents often results in superior advantages, offsetting disadvantages, and promoting therapeutic outcomes over monotherapy. Injectable biomaterials, either natural or synthetic, as in situ forming degradable networks not only can decrease patient discomfort and costs but also can act as new and good candidates for minimally invasive surgery. The capability of biomaterials for the design and fabrication of injectable systems strongly depends on their physicochemical and mechanical properties. The design and fabrication of injectable systems containing cells, therapeutic molecules, particles, and biomolecules that can be injected into the geometrically complex regions of the body tissues present a major challenge since they must ensure the maintenance of bioactivity of drugs/biomolecules/materials as well as survival and retention of cells. In this scenario, the high capacity of hydrogels for manipulation, encapsulation, and co-delivery of therapeutic molecules, cells, biomolecules, and nanomaterials make them excellent choices to address such challenges. One of the advantages of hydrogels is that the mechanical and degradation properties of these systems can be tuned by changing the 


\section{WILEY-VCH}

cross-linking method and chemical composition. The ability for tailoring the mechanical strength of IHs provides a possibility for in situ co-encapsulation of therapeutic molecules, cells, nanomaterials, and growth factors into the matrix for multimodal synergistic therapies.

Several challenges and unsolved scientific issues remain to be addressed in the future to increase the translation chance of IHs into the routine clinic. First, future assessments for using IHs towards multimodal synergistic therapies should be implemented in the large animal models such as monkeys and dogs, or even ex-vivo human tissue models. Furthermore, to achieve accurate and precise results that can be translated into clinical trials, the period of the in vivo assessments should be extended from weeks to several months. On one hand, next consideration is related to some of the crosslinking agents for IH formation that exhibit high toxicity, and hence the residues will cause undesirable responses in-vivo. On the other hand, toxic crosslinkers might interact with therapeutic molecules/biomolecules or nanomaterials encapsulated into the matrix of hydrogels that, in turn, result in their bioactivity loss. Likewise, sol-gel transition of IHs is another fundamental issue that needs much attention. A quick sol-gel transition of the precursor solutions might create the stuck of the fluid in needle, while the precursor solutions with high viscosity need high injection force, which in turn confer hand fatigue to clinicians and inconveniency to patients. Rapid drug release and degradation rate are other issues that should be taken into account for the clinical translation of the IHs. Degradation rate plays an important role in controlling the release of therapeutic agents and tissue regeneration. The fast degradation of hydrogel might trigger acute inflammatory response due to degradation products, whereas slow degradation might give rise to the insufficient release of therapeutic agents. Therefore, the degradation rate must be tuned by varying composition, topology, and crystallinity of polymers. More understanding of the physiochemical properties of polymers will help expert researchers to 


\section{WILEY-VCH}

address these challenges. Overall, the future design of IHs should be more focused on creating simple and well-defined 3D networks with minimal toxicity, optimized biodegradation rate, and proper functionality.

\section{Acknowledgements}

H. A. Santos acknowledges the financial support from the HiLIFE Research Funds, and the

Sigrid Jusélius Foundation. M.-A. Shahbazi acknowledges the financial support from the Academy of Finland (grant no. 317316).

\section{Conflict of Interest}

The authors declare no conflict of interest. 


\section{References}

[1] P. Mehta, D. F. McAuley, M. Brown, E. Sanchez, R. S. Tattersall, J. J. Manson, The Lancet 2020, 395, 1033.

[2] P. Davoodi, W. C. Ng, M. P. Srinivasan, C. H. Wang, Biotechnol. Bioeng. 2017, 114, 2931.

[3] Z. Gu, S. Zhu, L. Yan, F. Zhao, Y. Zhao, Adv. Mater. 2019, 31, 1800662.

[4] G. Tian, X. Zhang, Z. Gu, Y. Zhao, Adv. Mater. 2015, 27, 7692; W. Fan, B. Yung, P. Huang, X. Chen, Chem. Rev. 2017, 117, 13566.

[5] W. Fan, W. Bu, J. Shi, Adv. Mater. 2016, 28, 3987.

[6] M. Motwani, T. M. Delohery, G. K. Schwartz, Clin. Cancer Res. 1999, 5, 1876.

[7] Y. Akiyama, Y. Kimura, R. Enatsu, T. Mikami, M. Wanibuchi, N. Mikuni, World Neurosurg 2018, 113, e508; C. Y. X. Chua, J. Ho, S. Demaria, M. Ferrari, A. Grattoni, Adv. Ther. 2020, 2000027.

[8] C. Bastiancich, P. Danhier, V. Préat, F. Danhier, J Control Release 2016, 243, 29; S. A. Limentani, A. Asher, M. Heafner, J. W. Kim, R. Fraser, J. Neurooncol. 2005, 72, 241; L. S. Ashby, K. A. Smith, B. Stea, World J. Surg. Oncol. 2016, 14, 225.

[9] P. Majumder, U. Baxa, S. T. Walsh, J. P. Schneider, Angew. Chem. Int. Ed. 2018, 57, 15040; C. Wang, J. Wang, X. Zhang, S. Yu, D. Wen, Q. Hu, Y. Ye, H. Bomba, X. Hu, Z. Liu, Sci. Transl. Med. 2018, 10.

[10] X. Zhao, Y. Liang, Y. Huang, J. He, Y. Han, B. Guo, Adv. Funct. Mater. 2020, 30, 1910748; J. Qu, X. Zhao, P. X. Ma, B. Guo, Acta Biomater. 2018, 72, 55; J. Qu, X. Zhao, P. X. Ma, B. Guo, Acta Biomater. 2017, 58, 168; Y. Liang, B. Chen, M. Li, J. He, Z. Yin, B. Guo, Biomacromolecules 2020, 21, 1841.

[11] T. Thambi, Y. Li, D. S. Lee, J Control Release 2017, 267, 57.

[12] H. Amani, H. Arzaghi, M. Bayandori, A. S. Dezfuli, H. Pazoki-Toroudi, A. Shafiee, L. Moradi, Adv. Mater. Interfaces 2019, 6, 1900572; J. Yang, Z. Shi, R. Liu, Y. Wu, X. Zhang, Theranostics 2020, 10, 3223; M.-A. Shahbazi, L. Faghfouri, M. P. Ferreira, P. Figueiredo, H. Maleki, F. Sefat, J. Hirvonen, H. A. Santos, Chem. Soc. Rev. 2020, 49, 1253.

[13] L. Yu, J. Ding, Chem. Soc. Rev. 2008, 37, 1473; J. Qu, X. Zhao, Y. Liang, T. Zhang, P. X. Ma, B. Guo, Biomaterials 2018, 183, 185.

[14] B. Guo, J. Qu, X. Zhao, M. Zhang, Acta Biomater. 2019, 84, 180.

[15] B.-S. Kim, C.-S. Cho, J Tissue Eng Regen Med 2018, 15, 511; J. Radhakrishnan, A. Subramanian, U. M. Krishnan, S. Sethuraman, Biomacromolecules 2017, 18, 1; M. R. Matanović, J. Kristl, P. A. Grabnar, Int. J. Pharm. 2014, 472, 262.

[16] R. Dimatteo, N. J. Darling, T. Segura, Adv. Drug Deliv. Rev. 2018, 127, 167.

[17] B. Balakrishnan, N. Joshi, A. Jayakrishnan, R. Banerjee, Acta Biomater. 2014, 10, 3650; K. Ren, C. He, C. Xiao, G. Li, X. Chen, Biomaterials 2015, 51, 238; Y. Xu, Y. Xu, B. Bi, M. Hou, L. Yao, Q. Du, A. He, Y. Liu, C. Miao, X. Liang, Acta Biomater. 2020.

[18] L. Cao, B. Cao, C. Lu, G. Wang, L. Yu, J. Ding, J Mater Chem B 2015, 3, 1268.

[19] T. C. Tseng, L. Tao, F. Y. Hsieh, Y. Wei, I. M. Chiu, S. h. Hsu, Adv. Mater. 2015, 27, 3518.

[20] P. Du, A. D. S. Da Costa, C. Savitri, S. S. Ha, P.-Y. Wang, K. Park, Mater. Sci. Eng. C 2020, 110961; A. Pal, C. I. Smith, J. Palade, S. Nagaraju, B. A. Alarcon-Benedetto, J. Kilbourne, A. Rawls, J. Wilson-Rawls, B. L. Vernon, M. Nikkhah, Acta Biomater. 2020.

[21] F. Z. Kocak, A. Talari, M. Yar, I. U. Rehman, Int. J. Mol. Sci. 2020, 21, 1633.

[22] A. Sivashanmugam, R. A. Kumar, M. V. Priya, S. V. Nair, R. Jayakumar, Eur. Polym. J. 2015, 72, 543; C. D. Moreira, S. M. Carvalho, H. S. Mansur, M. M. Pereira, Mater. Sci. Eng. C 2016, 58, 1207; W. Liu, J. Sun, Y. Sun, Y. Xiang, Y. Yan, Z. Han, W. Bi, F. Yang, Q. Zhou, L. Wang, Chem. Eng. J. 2020, 124875.

[23] A. Tuladhar, J. M. Obermeyer, S. L. Payne, R. C. Siu, S. Zand, C. M. Morshead, M. S. Shoichet, Biomaterials 2020, 119794.

[24] A. M. Sisso, M. O. Boit, C. A. Deforest, J Biomed Mater Res A 2020, 108, 1112; A. Biswas, T. Ghosh, P. K. Gavel, A. K. Das, ACS Appl. Bio Mater. 2020. 
[25] D. R. Griffin, W. M. Weaver, P. O. Scumpia, D. Di Carlo, T. Segura, Nat. Mater. 2015, 14, 737; J. Li, F. Yu, G. Chen, J. Liu, X.-L. Li, B. Cheng, X.-M. Mo, C. Chen, J.-F. Pan, ACS Appl.Mater. Interfaces 2020; A. Dev, S. J. Mohanbhai, A. C. Kushwaha, A. Sood, M. N. Sardoiwala, S. R. Choudhury, S. Karmakar, Acta Biomater. 2020.

[26] X. Zhao, H. Wu, B. Guo, R. Dong, Y. Qiu, P. X. Ma, Biomaterials 2017, 122, 34.

[27] J.-A. Yang, J. Yeom, B. W. Hwang, A. S. Hoffman, S. K. Hahn, Prog. Polym. Sci. 2014, 39, 1973.

[28] M. Liu, X. Zeng, C. Ma, H. Yi, Z. Ali, X. Mou, S. Li, Y. Deng, N. He, Bone Res. 2017, 5, 1; Y. Chao, Q. Chen, Z. Liu, Adv. Funct. Mater. 2020, 30, 1902785.

[29] K. E. Papathanasiou, P. Turhanen, S. I. Brückner, E. Brunner, K. D. Demadis, Sci. Rep. 2017, 7, 1.

[30] G. Cirillo, U. G. Spizzirri, M. Curcio, F. P. Nicoletta, F. lemma, Pharmaceutics 2019, 11, 486.

[31] A. G. Sontyana, A. P. Mathew, K.-H. Cho, S. Uthaman, I.-K. Park, J Tissue Eng Regen Med 2018, 15,575 .

[32] K. Lavanya, S. V. Chandran, K. Balagangadharan, N. Selvamurugan, Mater. Sci. Eng. C 2020, 110862; R. Mavlyanova, R. Yang, T. Tao, M. Aquib, S. Kesse, M. B. J. Maviah, K. O. Boakye-Yiadom, M. A. Farooq, B. Wang, Polym Adv Technol 2019.

[33] S. E. Kudaibergenov, N. Nuraje, Polymers 2018, 10, 1146.

[34] H.-D. Wu, J.-C. Yang, T. Tsai, D.-Y. Ji, W.-J. Chang, C.-C. Chen, S.-Y. Lee, Carbohydr. Polym. 2011, $85,318$.

[35] D.-Y. Ji, T.-F. Kuo, H.-D. Wu, J.-C. Yang, S.-Y. Lee, Carbohydr. Polym. 2012, 89, 1123.

[36] T. M. D. Le, H. T. T. Duong, T. Thambi, V. Giang Phan, J. H. Jeong, D. S. Lee, Biomacromolecules 2018, 19, 3536.

[37] D. A. Gyles, L. D. Castro, J. O. C. Silva Jr, R. M. Ribeiro-Costa, Eur. Polym. J. 2017, 88, 373.

[38] K. Saekhor, W. Udomsinprasert, S. Honsawek, W. Tachaboonyakiat, Int. J. Biol. Macromol. 2019, $123,167$.

[39] W.-R. Zhuang, Y. Wang, P.-F. Cui, L. Xing, J. Lee, D. Kim, H.-L. Jiang, Y.-K. Oh, J Control Release 2019, 294, 311.

[40] Y.-C. Chang, Y.-D. Chen, C.-H. Chen, Y.-S. Wen, J. T. Lin, H.-Y. Chen, M.-Y. Kuo, I. Chao, J. Org. Chem. 2008, 73, 4608.

[41] A. Mahler, M. Reches, M. Rechter, S. Cohen, E. Gazit, Adv. Mater. 2006, 18, 1365.

[42] X. Dai, Y. Zhang, L. Gao, T. Bai, W. Wang, Y. Cui, W. Liu, Adv. Mater. 2015, 27, 3566.

[43] P. Wang, J. Zhang, Y. Li, N. Wang, W. Liu, Mater. Lett. 2015, 142, 71.

[44] X. Zhai, Y. Ma, C. Hou, F. Gao, Y. Zhang, C. Ruan, H. Pan, W. W. Lu, W. Liu, ACS Biomater. Sci. Eng. 2017, 3, 1109.

[45] P. Y. Dankers, M. J. van Luyn, A. Huizinga-van der Vlag, G. M. van Gemert, A. H. Petersen, E. Meijer, H. M. Janssen, A. W. Bosman, E. R. Popa, Biomaterials 2012, 33, 5144.

[46] P. Y. Dankers, T. M. Hermans, T. W. Baughman, Y. Kamikawa, R. E. Kieltyka, M. M. Bastings, H. M. Janssen, N. A. Sommerdijk, A. Larsen, M. J. Van Luyn, Adv. Mater. 2012, 24, 2703; M. M. Bastings, S. Koudstaal, R. E. Kieltyka, Y. Nakano, A. Pape, D. A. Feyen, F. J. Van Slochteren, P. A. Doevendans, J. P. Sluijter, E. Meijer, Adv. Healthc. Mater. 2014, 3, 70.

[47] X. Ding, Y. Wang, J Mater Chem B 2017, 5, 887.

[48] Z. Deng, Y. Guo, X. Zhao, P. X. Ma, B. Guo, Chem. Mater. 2018, 30, 1729.

[49] J. E. Mealy, C. B. Rodell, J. A. Burdick, J Mater Chem B 2015, 3, 8010; G. Liu, Q. Yuan, G. Hollett, W. Zhao, Y. Kang, J. Wu, Polym. Chem. 2018, 9, 3436.

[50] Y. Wu, B. Guo, P. X. Ma, ACS Macro Lett. 2014, 3, 1145; C. T. Huynh, M. K. Nguyen, D. S. Lee, Macromolecules 2011, 44, 6629.

[51] J. Gopinathan, I. Noh, J Tissue Eng Regen Med 2018, 15, 531.

[52] H. S. Abandansari, M. H. Ghanian, F. Varzideh, E. Mahmoudi, S. Rajabi, P. Taheri, M. R. Nabid, H. Baharvand, Biomaterials 2018, 170, 12. 


\section{WILEY-VCH}

[53] N. Yang, Y. Wang, Q. Zhang, L. Chen, Y. Zhao, J. Mater. Sci. 2019, 54, 1677; C. A. DeForest, B. D. Polizzotti, K. S. Anseth, Nat. Mater. 2009, 8, 659.

[54] L. J. Macdougall, V. X. Truong, A. P. Dove, ACS Macro Lett. 2017, 6, 93.

[55] K. S. Lim, B. J. Klotz, G. C. Lindberg, F. P. Melchels, G. J. Hooper, J. Malda, D. Gawlitta, T. B. Woodfield, Macromol. Biosci. 2019, 19, 1900098; J. A. Burdick, K. S. Anseth, Biomaterials 2002, 23, 4315.

[56] J. L. Ifkovits, J. A. Burdick, Tissue Eng. 2007, 13, 2369.

[57] H. Tan, K. G. Marra, Materials 2010, 3, 1746.

[58] S.-J. Yoon, Y. Yoo, S. E. Nam, H. Hyun, D.-W. Lee, S. Um, S. Y. Kim, S. O. Hong, D. H. Yang, H. J. Chun, Mar. Drugs. 2018, 16, 351.

[59] S.-J. Yoon, H. Hyun, D.-W. Lee, D. H. Yang, Molecules 2017, 22, 1513; H. Hyun, M. H. Park, W. Lim, S. Y. Kim, D. Jo, J. S. Jung, G. Jo, S. Um, D.-W. Lee, D. H. Yang, Artif Cells Nanomed Biotechnol 2018, $46,874$.

[60] R. Jin, L. M. Teixeira, A. Krouwels, P. J. Dijkstra, C. Van Blitterswijk, M. Karperien, J. Feijen, Acta Biomater. 2010, 6, 1968.

[61] Y. Lee, H. J. Chung, S. Yeo, C.-H. Ahn, H. Lee, P. B. Messersmith, T. G. Park, Soft Matter 2010, 6, 977.

[62] Y. Dong, M. Rodrigues, X. Li, S. H. Kwon, N. Kosaric, S. Khong, Y. Gao, W. Wang, G. C. Gurtner, Adv. Funct. Mater. 2017, 27, 1606619.

[63] E. Bakaic, N. M. Smeets, T. Hoare, RSC Adv. 2015, 5, 35469.

[64] M. P. Lutolf, G. P. Raeber, A. H. Zisch, N. Tirelli, J. A. Hubbell, Adv. Mater. 2003, 15, 888; J. Patterson, J. A. Hubbell, Biomaterials 2011, 32, 1301; J. Patterson, J. A. Hubbell, Biomaterials 2010, 31, 7836; Y. S. Jo, S. C. Rizzi, M. Ehrbar, F. E. Weber, J. A. Hubbell, M. P. Lutolf, J Biomed Mater Res A 2010, $93,870$.

[65] J. Xu, Y. Liu, S.-h. Hsu, Molecules 2019, 24, 3005.

[66] Z. Zhang, C. He, X. Chen, Mater. Chem. Front. 2018, 2, 1765.

[67] S. Yan, T. Wang, L. Feng, J. Zhu, K. Zhang, X. Chen, L. Cui, J. Yin, Biomacromolecules 2014, 15, 4495.

[68] X. Wang, S. Chen, D. Wu, Q. Wu, Q. Wei, B. He, Q. Lu, Q. Wang, Adv. Mater. 2018, 30, 1705668.

[69] K. Kuwahara, Z. Yang, G. C. Slack, M. E. Nimni, B. Han, Tissue Eng Part C Methods 2010, 16, 609.

[70] A. Ranga, M. P. Lutolf, J. n. Hilborn, D. A. Ossipov, Biomacromolecules 2016, 17, 1553.

[71] R. Jin, B. Lou, C. Lin, Polym. Int. 2013, 62, 353.

[72] L. Wang, J. Li, D. Zhang, S. Ma, J. Zhang, F. Gao, F. Guan, M. Yao, RSC Adv. 2020, 10, 2870; M. Yao, F. Gao, R. Xu, J. Zhang, Y. Chen, F. Guan, Biomater. Sci. 2019, 7, 4088.

[73] L. S. M. Teixeira, J. Feijen, C. A. van Blitterswijk, P. J. Dijkstra, M. Karperien, Biomaterials 2012, $33,1281$.

[74] T. B. Ramos, T. Feijão, A. Gonçalves, M. A. Shahbazi, Z. Liu, C. Barrias, M. J. Oliveira, P. Granja, H. A. Santos, B. Sarmento, J Control Release 2020.

[75] T. Bauleth-Ramos, T. Y. Shih, M. A. Shahbazi, A. J. Najibi, A. S. Mao, D. Liu, P. Granja, H. A. Santos, B. Sarmento, D. J. Mooney, Adv. Funct. Mater. 2019, 29, 1903686.

[76] R. B. Mokhtari, T. S. Homayouni, N. Baluch, E. Morgatskaya, S. Kumar, B. Das, H. Yeger, Oncotarget 2017, 8, 38022 .

[77] X. Liu, Z. Li, X. J. Loh, K. Chen, Z. Li, Y. L. Wu, Macromol. Rapid Commun. 2019, 40, 1800117.

[78] W. Shen, X. Chen, J. Luan, D. Wang, L. Yu, J. Ding, ACS Appl.Mater. Interfaces 2017, 9, 40031.

[79] M. He, J. Sui, Y. Chen, S. Bian, Y. Cui, C. Zhou, Y. Sun, J. Liang, Y. Fan, X. Zhang, J Mater Chem B 2017, 5, 4852.

[80] P. S. Yavvari, S. Pal, S. Kumar, A. Kar, A. K. Awasthi, A. Naaz, A. Srivastava, A. Bajaj, ACS Biomater. Sci. Eng. 2017, 3, 3404. 
[81] W. Xie, Q. Gao, Z. Guo, D. Wang, F. Gao, X. Wang, Y. Wei, L. Zhao, ACS Appl.Mater. Interfaces 2017, 9, 33660.

[82] R. Xing, K. Liu, T. Jiao, N. Zhang, K. Ma, R. Zhang, Q. Zou, G. Ma, X. Yan, Adv. Mater. 2016, 28, 3669.

[83] X. Xu, Z. Zeng, Z. Huang, Y. Sun, Y. Huang, J. Chen, J. Ye, H. Yang, C. Yang, C. Zhao, Carbohydr. Polym. 2020, 229, 115394.

[84] S. Gou, D. Xie, Y. Ma, Y. Huang, F. Dai, C. Wang, B. Xiao, ACS Biomater. Sci. Eng. 2019.

[85] S. Gou, D. Xie, Y. Ma, Y. Huang, F. Dai, C. Wang, B. Xiao, ACS Biomater. Sci. Eng. 2019, 6, 1052.

[86] Y.-W. Jiang, G. Gao, P. Hu, J.-B. Liu, Y. Guo, X. Zhang, X.-W. Yu, F.-G. Wu, X. Lu, Nanoscale 2020, $12,210$.

[87] J. Zhou, M. Wang, Y. Han, J. Lai, J. Chen, ACS Appl. Bio Mater. 2019.

[88] Y. Zheng, W. Wang, J. Zhao, C. Wu, C. Ye, M. Huang, S. Wang, Carbohydr. Polym. 2019, 222, 115039.

[89] C. Liu, X. Guo, C. Ruan, H. Hu, B.-P. Jiang, H. Liang, X.-C. Shen, Acta Biomater. 2019, 96, 281.

[90] R. Jin, J. Yang, D. Zhao, X. Hou, C. Li, W. Chen, Y. Zhao, Z. Yin, B. Liu, J. Nanobiotechnology 2019, $17,1$.

[91] R. Jin, X. Yang, D. Zhao, X. Hou, C. Li, X. Song, W. Chen, Q. Wang, Y. Zhao, B. Liu, Nanoscale 2019, $11,16080$.

[92] J. Wan, S. Geng, H. Zhao, X. Peng, J. Xu, M. Wei, J. Mao, Y. Zhou, Q. Zhu, Y. Zhao, Nanoscale 2018, 10, 20020.

[93] G. He, S. Chen, Y. Xu, Z. Miao, Y. Ma, H. Qian, Y. Lu, Z. Zha, Mater. Horiz. 2019, 6, 711.

[94] M. Hou, R. Yang, L. Zhang, L. Zhang, G. Liu, Z. Xu, Y. Kang, P. Xue, ACS Biomater. Sci. Eng. 2018, 4, 4266; H. Fan, L. Wang, X. Feng, Y. Bu, D. Wu, Z. Jin, Macromolecules 2017, 50, 666; J. Deng, X. Xun, W. Zheng, Y. Su, L. Zheng, C. Wang, M. Su, J Mater Chem B 2018, 6, 7966.

[95] Z.-Q. Zhang, S.-C. Song, Biomaterials 2017, 132, 16.

[96] J. Zhao, J. Li, C. Zhu, F. Hu, H. Wu, X. Man, Z. Li, C. Ye, D. Zou, S. Wang, ACS Appl.Mater. Interfaces 2018, 10, 3392.

[97] P. d. I. Puente, N. Fettig, M. J. Luderer, A. Jin, S. Shah, B. Muz, V. Kapoor, S. M. Goddu, N. N. Salama, C. Tsien, D. Thotala, K. Shoghi, B. Rogers, A. K. Azab, J. Pharm. Sci. 2018, 107, 922.

[98] E. Mei, S. Li, J. Song, R. Xing, Z. Li, X. Yan, Colloids Surf. A Physicochem. Eng. Asp. 2019, 577, 570.

[99] S. Das, A. B. Baker, Front. Bioeng. Biotechnol. 2016, 4, 82; B. Zhang, J. He, M. Shi, Y. Liang, B. Guo, Chem. Eng. J. 2020, 400, 125994.

[100] J. Qu, X. Zhao, Y. Liang, Y. Xu, P. X. Ma, B. Guo, Chem. Eng. J. 2019, 362, 548.

[101] H. Chen, R. Cheng, X. Zhao, Y. Zhang, A. Tam, Y. Yan, H. Shen, Y. S. Zhang, J. Qi, Y. Feng, NPG Asia Mater. 2019, 11, 1.

[102] L. Kong, Z. Wu, H. Zhao, H. Cui, J. Shen, J. Chang, H. Li, Y. He, ACS Appl.Mater. Interfaces 2018, 10, 30103.

[103] Y. Li, Y. Han, X. Wang, J. Peng, Y. Xu, J. Chang, ACS Appl.Mater. Interfaces 2017, 9, 16054.

[104] C. Loebel, C. B. Rodell, M. H. Chen, J. A. Burdick, Nat. Protoc. 2017, 12, 1521; Y. Tu, N. Chen, C. Li, H. Liu, R. Zhu, S. Chen, Q. Xiao, J. Liu, S. Ramakrishna, L. He, Acta Biomater. 2019, 90, 1.

[105] M. Chen, J. Tian, Y. Liu, H. Cao, R. Li, J. Wang, J. Wu, Q. Zhang, Chem. Eng. J. 2019, 373, 413.

[106] S. J. Bidarra, C. C. Barrias, P. L. Granja, Acta Biomater. 2014, 10, 1646; C. Yan, M. E. Mackay, K. Czymmek, R. P. Nagarkar, J. P. Schneider, D. J. Pochan, Langmuir 2012, 28, 6076.

[107] L. Zhao, L. Niu, H. Liang, H. Tan, C. Liu, F. Zhu, ACS Appl.Mater. Interfaces 2017, 9, 37563.

[108] S.-h. Hsu, G.-S. Huang, Biomaterials 2013, 34, 4725; G. Chen, Y. Qi, L. Niu, T. Di, J. Zhong, T. Fang, W. Yan, Biomed Rep 2015, 3, 749.

[109] H. Amani, E. Mostafavi, H. Arzaghi, S. Davaran, A. Akbarzadeh, O. Akhavan, H. Pazoki-Toroudi, T. J. Webster, ACS Biomater. Sci. Eng. 2018, 5, 193. 
[110] G. Reina, J. M. González-Domínguez, A. Criado, E. Vázquez, A. Bianco, M. Prato, Chem. Soc. Rev. 2017, 46, 4400.

[111] X. Sun, J. Su, J. Bao, T. Peng, L. Zhang, Y. Zhang, Y. Yang, X. Zhou, Biomaterials 2012, 33, 8265.

[112] W. Kafienah, F. Al-Fayez, A. P. Hollander, M. D. Barker, Arthritis Rheumatol. 2003, 48, 709.

[113] H. Sá-Lima, S. G. Caridade, J. F. Mano, R. L. Reis, Soft Matter 2010, 6, 5184.

[114] K. Yun, H. T. Moon, J. Biosci. Bioeng. 2008, 105, 122.

[115] A. Arora, A. Mahajan, D. S. Katti, Colloids Surf. B 2017, 159, 838.

[116] V. F. Segers, R. T. Lee, Nature 2008, 451, 937; L. C. Amado, A. P. Saliaris, K. H. Schuleri, M. S. John, J.-S. Xie, S. Cattaneo, D. J. Durand, T. Fitton, J. Q. Kuang, G. Stewart, Proc. Natl. Acad. Sci. U.S.A. 2005, 102, 11474; Y. Miyahara, N. Nagaya, M. Kataoka, B. Yanagawa, K. Tanaka, H. Hao, K. Ishino, H. Ishida, T. Shimizu, K. Kangawa, Nat. Med. 2006, 12, 459.

[117] M. Habib, K. Shapira-Schweitzer, O. Caspi, A. Gepstein, G. Arbel, D. Aronson, D. Seliktar, L. Gepstein, Biomaterials 2011, 32, 7514.

[118] M. Song, H. Jang, J. Lee, J. H. Kim, S. H. Kim, K. Sun, Y. Park, Biomaterials 2014, 35, 2436.

[119] T. Hao, J. Li, F. Yao, D. Dong, Y. Wang, B. Yang, C. Wang, ACS nano 2017, 11, 5474.

[120] C. N. Kotanen, A. N. Wilson, C. Dong, C.-Z. Dinu, G. A. Justin, A. Guiseppi-Elie, Biomaterials 2013, 34, 6318; B. Guo, P. X. Ma, Biomacromolecules 2018, 19, 1764.

[121] A. B. Mathur, A. M. Collinsworth, W. M. Reichert, W. E. Kraus, G. A. Truskey, J. Biomech. 2001, 34, 1545; H. L. Granzier, T. C. Irving, Biophys. J. 1995, 68, 1027.

[122] W. Wang, B. Tan, J. Chen, R. Bao, X. Zhang, S. Liang, Y. Shang, W. Liang, Y. Cui, G. Fan, Biomaterials 2018, 160, 69.

[123] R. Dong, X. Zhao, B. Guo, P. X. Ma, ACS Appl.Mater. Interfaces 2016, 8, 17138.

[124] Y. Liu, J. K. Chan, S. H. Teoh, J Tissue Eng Regen Med 2015, 9, 85.

[125] H. Oliveira, S. Catros, O. Castano, S. Rey, R. Siadous, D. Clift, J. Marti-Munoz, M. Batista, R. Bareille, J. Planell, Acta Biomater. 2017, 54, 377.

[126] L. Liu, Y. Xiang, Z. Wang, X. Yang, X. Yu, Y. Lu, L. Deng, W. Cui, NPG Asia Mater. 2019, 11, 1.

[127] W. Pan, C. Dai, Y. Li, Y. Yin, L. Gong, J. O. a. Machuki, Y. Yang, S. Qiu, K. Guo, F. Gao, Biomaterials 2020, 239, 119851.

[128] A. Parameswaran-Thankam, C. M. Parnell, F. Watanabe, A. B. RanguMagar, B. P. Chhetri, P. K. Szwedo, A. S. Biris, A. Ghosh, ACS omega 2018, 3, 15158.

[129] W. Wang, M. Dang, Z. Zhang, J. Hu, T. W. Eyster, L. Ni, P. X. Ma, Acta Biomater. 2016, 36, 63; B. Chang, N. Ahuja, C. Ma, X. Liu, Mater. Sci. Eng. R Rep. 2017, 111, 1.

[130] X. Xu, Z. Gu, X. Chen, C. Shi, C. Liu, M. Liu, L. Wang, M. Sun, K. Zhang, Q. Liu, Acta Biomater. 2019, 86, 235.

[131] J. Tan, M. Zhang, Z. Hai, C. Wu, J. Lin, W. Kuang, H. Tang, Y. Huang, X. Chen, G. Liang, Acs Nano 2019, 13, 5616.

[132] C. R. Silva, P. S. Babo, M. Gulino, L. Costa, J. M. Oliveira, J. Silva-Correia, R. M. Domingues, R. L. Reis, M. E. Gomes, Acta Biomater. 2018, 77, 155.

[133] Z. Wei, J. Zhao, Y. M. Chen, P. Zhang, Q. Zhang, Sci. Rep. 2016, 6, 37841.

[134] Y.-Z. Zhao, X. Jiang, J. Xiao, Q. Lin, W.-Z. Yu, F.-R. Tian, K.-L. Mao, W. Yang, H. L. Wong, C.-T. Lu, Acta Biomater. 2016, 29, 71.

[135] Y. Z. Zhao, X. Jiang, Q. Lin, H. L. Xu, Y. D. Huang, C. T. Lu, J. Cai, J Biomed Mater Res A 2017, 105, 2816.

[136] R. Li, Y. Li, Y. Wu, Y. Zhao, H. Chen, Y. Yuan, K. Xu, H. Zhang, Y. Lu, J. Wang, Biomaterials 2018, $168,24$.

[137] P. Moshayedi, L. R. Nih, I. L. Llorente, A. R. Berg, J. Cinkornpumin, W. E. Lowry, T. Segura, S. T. Carmichael, Biomaterials 2016, 105, 145.

[138] A. K. Gaharwar, I. Singh, A. Khademhosseini, Nat. Rev. Mater. 2020, 1. 


\section{WILEY-VCH}

[139] L. R. Nih, S. Gojgini, S. T. Carmichael, T. Segura, Nat. Mater. 2018, 17, 642.

[140] M. Boffito, C. Pontremoli, S. Fiorilli, R. Laurano, G. Ciardelli, C. Vitale-Brovarone, Pharmaceutics 2019, 11, 501.

[141] A. Tuladhar, J. M. Obermeyer, S. L. Payne, R. C. Siu, S. Zand, C. M. Morshead, M. S. Shoichet, Biomaterials 2020, 235, 119794.

[142] M. Fan, Y. Ma, H. Tan, Y. Jia, S. Zou, S. Guo, M. Zhao, H. Huang, Z. Ling, Y. Chen, Mater. Sci. Eng. C 2017, 71, 67.

[143] A. J. Rufaihah, N. A. Johari, S. R. Vaibavi, M. Plotkin, T. Kofidis, D. Seliktar, Acta Biomater. 2017, $48,58$.

[144] Y. Zhang, C. Fu, Y. Li, K. Wang, X. Wang, Y. Wei, L. Tao, Polym. Chem. 2017, 8, 537.

[145] S. Pentlavalli, P. Chambers, B. N. Sathy, M. O'Doherty, M. Chalanqui, D. J. Kelly, T. HautDonahue, H. O. McCarthy, N. J. Dunne, Macromol Biosci 2017, 17, 1700118.

[146] N. T. B. Linh, C. D. Abueva, B.-T. Lee, Biomed Mater 2017, 12, 015026. 